\title{
Waste minimized cyanosilylation of carbonyls using fluoride on polymeric ionic-tags in batch and under continuous flow conditions
}

Francesco Ferlin, ${ }^{\text {ta }}$ Federica Valentini, ${ }^{\text {ta }}$ Giulia Brufani, ${ }^{\mathrm{a}}$ Daniela, Lanari, ${ }^{\text {b }}$ Luigi Vaccaro*a

a Laboratory of Green S.O.C. - Dipartimento di Chimica, Biologia e Biotecnologie, Università degli Studi di Perugia, Via Elce di Sotto 8, 06123 - Perugia - Italy;

E-mail: luigi.vaccaro@unipg.it; Web: www.dcbb.unipg.it/greensoc.

${ }^{b}$ Dipartimento di Scienze Farmaceutiche, Università degli Studi di Perugia, Via del Liceo 1, 06123 - Perugia - Italy;

$\ddagger$ Both authors contributed equally to this work.

e-mail: luigi.vaccaro@unipg.it daniela.lanari@unipg.it

total number of pages: 56

total numbers of tables: 3 at pages S3-4

total number of figures: 3 at pages S5-6

\section{Table of Contents:}

1. General Remarks

2. General Procedures

S2

3. Data used for Environmental Index and Safety Hazard Index

4. Radial polygon graphical representation for mass-related metrics

5. Characterization Data

6. ${ }^{1} \mathrm{H},{ }^{13} \mathrm{C}$ and ${ }^{19} \mathrm{~F}$ NMR Spectra 


\section{General Remarks}

All chemicals were used without any further purification unless otherwise noted. 4Vinylbenzylchloride (VBC) and styrene (STY) were extracted three times with a $10 \% \mathrm{w} / \mathrm{w} \mathrm{NaOH}$ solution and filtered through $\mathrm{Al}_{2} \mathrm{O}_{3}$ pad to remove the polymerization inhibitor (tert-butyl catechol). Dibenzoylperoxide was re-crystallized from methanol. GLC analyses were performed by using Agilent 5890 Series II GC System equipped with a capillary column DB-5MS (30 m, $0.32 \mathrm{~mm})$, a FID detector and helium as gas carrier. GC-EIMS analyses were carried out by using a Hewlett-Packard HP 6890N Network GC system/5975 Mass Selective Detector equipped with an electron impact ionizer at $70 \mathrm{eV} .{ }^{1} \mathrm{H},{ }^{13} \mathrm{C}$ and ${ }^{19} \mathrm{~F}$ NMR spectra were recorded on a Bruker DRX-ADVANCE $400 \mathrm{MHz}$ $\left({ }^{1} \mathrm{H}\right.$ at $400 \mathrm{MHz},{ }^{13} \mathrm{C}$ at $100.6 \mathrm{MHz}$ and ${ }^{19} \mathrm{~F}$ at $\left.376 \mathrm{MHz}\right)$. Chemical shifts are reported in ppm and coupling constants in Hertz. ATR-IR spectra were recorded on Bruker Optics ALPHA-P spectrometer, equipped with a Platinum ATR module. Elemental Analysis (EA) were conducted on Elementar UNICUBE® elemental analyzer.

3,3-bis $(1 \mathrm{H}$-imidazol-1-yl)propan-1-ol ligand was prepared according to previously reported procedure. $^{1}$

Characterization data, ${ }^{1} \mathrm{H},{ }^{13} \mathrm{C}$ and ${ }^{19} \mathrm{~F}$ NMR spectra are reported below.

\section{General Procedures}

Synthesis of SPACER (1,4-bis(4-vinylphenoxy)benzene). ${ }^{2}$ In a $250 \mathrm{~mL}$ round bottom flask equipped with a condenser and a magnetic stirrer, 4-fluorobenzaldehyde $(9.54 \mathrm{~g}, 76.8 \mathrm{mmol})$, hydroquinone $(4.62 \mathrm{~g}, 42.0 \mathrm{mmol})$, potassium carbonate $(14.52 \mathrm{~g}, 105.0 \mathrm{mmol})$ and dry DMF $(60.0 \mathrm{~mL})$ were added. The reaction mixture was left under stirring at $160^{\circ} \mathrm{C}$ for $6 \mathrm{~h}$, then poured into cold water and filtered to afford 4,4'-[1,4-phenylen-bis-(oxy)]dibenzaldehyde intermediate (12.0 g, $36.0 \mathrm{mmol}, 90 \%$ yield) as brown solid. In a $250 \mathrm{~mL}$ three-neck round bottom flask, equipped with a magnetic stirrer and an argon inlet/outlet, methyltriphenylphosphonium bromide $(6.7 \mathrm{~g}, 18.7 \mathrm{mmol})$ was dissolved in dry THF $(55 \mathrm{~mL})$ at $0{ }^{\circ} \mathrm{C}$. A solution of $\mathrm{nBuLi}(8.28 \mathrm{~mL}, 1.6 \mathrm{M}$ in $\mathrm{n}$-hexane, $13.23 \mathrm{mmol})$ was then added dropwise, and the reaction mixture was left under stirring at $0^{\circ} \mathrm{C}$ for $3 \mathrm{~h}$, then allowed to warm at room temperature. Next, a solution of 4,4'-[1,4-phenylenebis(oxy)]dibenzaldehyde $(2.0 \mathrm{~g}, 6.3$ $\mathrm{mmol})$ in dry THF $(15.0 \mathrm{~mL})$ was added, and this mixture was stirred at room temperature for an additional $24 \mathrm{~h}$. After this time, THF was removed under vacuum, and the residue was recrystallized from methanol, affording 1,4-bis(4-vinylphenoxy)benzene $(1.81 \mathrm{~g}, 5.8 \mathrm{mmol}, 92 \%$ yield).

Synthesis of SP02-CI supports. A three-neck cylinder-shaped glass vessel, equipped with a mechanical stirrer, a condenser, and a nitrogen inlet was charged with $120 \mathrm{~mL}$ of an aqueous $\mathrm{NaCl}$ (4 wt\%, $4.8 \mathrm{~g}$ ) solution containing acacia gum (8 wt\%, $9.6 \mathrm{~g})$. After deoxygenation a mixture of styrene (L: $5.62 \mathrm{~g}, 53.95 \mathrm{mmol}$; M: $2.98 \mathrm{~g}, 28.6 \mathrm{mmol}$ ), vinylbenzylchloride (L: $1.48 \mathrm{~g}, 9.75 \mathrm{mmol}$; M: $5.36 \mathrm{~g}, 35.1 \mathrm{mmol}), 1,4$-bis (4-vinylphenoxy)benzene $(0.409 \mathrm{~g}, 1.3 \mathrm{mmol})$, chlorobenzene $(8 \mathrm{~mL})$, and dibenzoylperoxide ( $2 \% \mathrm{wt} / \mathrm{wt}, 150 \mathrm{mg}, 0.62 \mathrm{mmol})$ was rapidly added to the solution. The suspension was kept under mechanical stirring at room temperature for an additional $30 \mathrm{~min}$, then heated at $85^{\circ} \mathrm{C}$ for $24 \mathrm{~h}$. Subsequently, the reaction mixture was cooled and the polymer beads were isolated by filtration, washed with water $(50 \mathrm{~mL})$, then extracted by using water $(100 \mathrm{~mL})$, THF $(100 \mathrm{~mL})$, and $\mathrm{n}$-hexane $(100 \mathrm{~mL})$ in a Soxhlet apparatus. The resulting SP02-Cl resins were dried under vacuum for $15 \mathrm{~h}$ at room temperature. The amount of $\mathrm{Cl}$ on polymer surface was determined by elemental analysis (L: $1.4 \mathrm{mmol} / \mathrm{g}, \mathrm{Cl}: 5.055 ; \mathbf{M}: 3.4 \mathrm{mmol} / \mathrm{g}, \mathrm{Cl}: 12.078$ ).

Synthesis of POLITAGs-I. A $25 \mathrm{~mL}$ two-neck round bottom flask equipped with magnetic stirrer and an argon inlet was charged with 3,3-bis(1 $\mathrm{H}$-imidazol-1-yl)propan-1-ol ligand (1.2 eq) in $10 \mathrm{~mL}$ of dry DMF. At $0^{\circ} \mathrm{C} \mathrm{NaH}(60 \%$ in mineral oil; $3 \mathrm{eq})$ was slowly added and the mixture was kept under stirring for $20 \mathrm{~min}$ and for additional $30 \mathrm{~min}$ at $28^{\circ} \mathrm{C}$. Then the resin SP02-Cl was added $(0.8 \mathrm{~g})$ and the 
mixture was kept under stirring at $60^{\circ} \mathrm{C}$ for $24 \mathrm{~h}$. The resulting material was washed with water and acetone and dried under vacuum before the subsequent treatment with methyliodide for the synthesis of supported bis-imidazolium salt. After $20 \mathrm{~h}$ at $90^{\circ} \mathrm{C}$ in presence of $5 \mathrm{~mL}$ of Mel, the resulting POLITAG-I was filtered and washed with methanol and acetone and dried under vacuum. Loading of bis-imidazolium units supported on polymer were determined by elemental analysis (POLITAG-L-I: C: 61.360; N: 3.09; H: 6.366; 0.55 mmol/g; POLITAG-M-I: C: 49.32; N: 5.63; H: $5.837 ; 1.01 \mathrm{mmol} / \mathrm{g})$

Synthesis of POLITAGs-F. ${ }^{3}$ POLITAG-I was charged onto a chromatographic column and an aqueous solution of KF (2M) was passed through the POLITAG-I until no silver iodide precipitate from the eluted solution. After the completion of the ionic exchange the resin was washed with acetone and dried under vacuum. Loading of bis-imidazolium units supported on polymer were determined by elemental analysis (POLITAG-L-F: C: $71.88 ; \mathrm{N}: 3.48 ; \mathrm{H}: 9.878 ; 0.62 \mathrm{mmol} / \mathrm{g}$; POLITAG-M-F: C: $68.64 ; \mathrm{N}: 7.43 ; \mathrm{H}: 8.353 ; 1.33 \mathrm{mmol} / \mathrm{g})$.

Typical batch procedure for cyanosilylation reaction. In a $2 \mathrm{~mL}$ screw capped vial were placed POLITAG-L-F (8 mg, $2 \mathrm{~mol} \%)$, carbonyl compound $(0.5 \mathrm{mmol})$ and TMSCN $(1.05 \mathrm{eq}, 66 \mu \mathrm{L})$. The mixture was kept under stirring at $60^{\circ} \mathrm{C}$ for the desired time. $1 \mathrm{~mL}$ of EtOAc was used for separating the catalyst and removed under reduced pressure to afford pure product.

Continuous Flow procedure for cyanosilylation reaction $(95 \mathrm{mmol} \mathrm{scale})$. Acetophenone (1a) $(\mathrm{d}=$ 1.03, $11.1 \mathrm{~mL}, 11.41 \mathrm{~g}, 95.00 \mathrm{mmol}$ ) and TMSCN (d = 0.793, $12.48 \mathrm{~mL}, 9.89 \mathrm{~g}, 99.75 \mathrm{mmol})$ were charged into a reservoir column installed in the inlet section of the HPLC pump (Waters model 590), while POLITAG-L-F (96 mg, $0.12 \mathrm{mmol} F, 0.62 \mathrm{mmol} / \mathrm{g}$ ) was dispersed in quartz fine granular and charged into a stainless steel column. The reaction mixture was pumped at a $250 \mu \mathrm{L} \mathrm{min}{ }^{-1}$ flow rate trough the catalyst at $60^{\circ} \mathrm{C}$ for $15 \mathrm{~h}$, and collected into a recovery flask. EtOAc $(0.5 \mathrm{~mL})$ was then cyclically pumped through the catalyst column to wash the system and the product 2a was recovered in $99 \%$ yield (oil, $20.8 \mathrm{~g}$ ) and with a $0.041 \mathrm{E}$-factor value (see below for calculation).

$E$-factor $($ flow $)=[11.41 \mathrm{~g}(\mathbf{1 a})+9.89 \mathrm{~g}($ TMSCN $)+0.096 \mathrm{~g}($ POLITAG-L-F $)+0.45 \mathrm{~g}(0.5 \mathrm{~mL}$ ethyl acetate cyclic for washing the system $)-(0.096 \mathrm{~g}($ POLITAG-L-F) $+20.83 \mathrm{~g} \mathrm{(2a)}) / 20.83(\mathbf{2 a})=0.04$

The same procedure has been used to synthesize compounds $\mathbf{2 f}, \mathbf{2} \mathbf{g}, \mathbf{2} \mathbf{h}$, and $\mathbf{6} \mathbf{d}$

Table S1. loading of different chloromethylated supports SP02-L-CI and SP02-M-CI

\begin{tabular}{|c|c|c|c|c|}
\hline & SP & STY & $\begin{array}{c}\underset{\mathrm{H}_{2} \mathrm{O} \text {, acacia gum, }}{\mathrm{NaCl}} \\
24 \mathrm{hC}, 85^{\circ} \mathrm{C}\end{array}$ & $\begin{array}{l}\text { SP } \\
\text { SP02-X-Cl } \\
\begin{array}{c}\mathrm{X}: \mathrm{M}=\text { medium } \\
\mathrm{L}=\text { low }\end{array}\end{array}$ \\
\hline SP02 & $\begin{array}{l}\text { loading } \mathrm{Cl} \\
(\mathrm{mmol} / \mathrm{g})\end{array}$ & SP (\%) & STY (\%) & VBC (\%) \\
\hline $\mathbf{L}$ & 1.4 & 2 & 83 & 15 \\
\hline M & 3.4 & 2 & 48 & 50 \\
\hline
\end{tabular}


Table S2. Catalytic tests for mechanism investigation. ${ }^{a}$

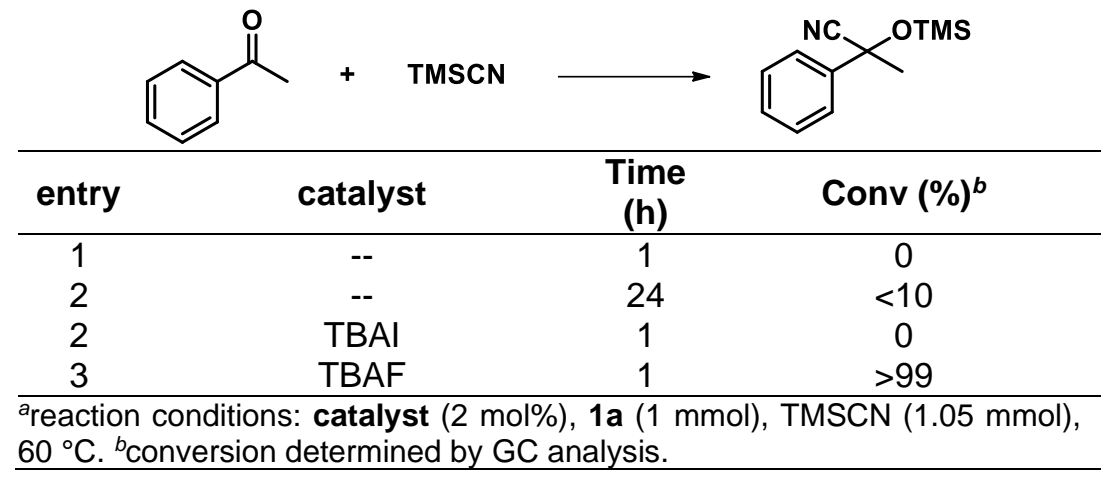

Table S3. optimization of reaction condition for cyanosilylation of 1a with POLITAG-L-F.a

\begin{tabular}{|c|c|c|c|c|}
\hline entry & BPR (psi) & $\begin{array}{l}\text { Flow rate } \\
\text { ( } \mu \mathrm{L} / \mathrm{min})\end{array}$ & $\begin{array}{l}\text { Residence } \\
\text { time (min) }\end{array}$ & Conv $(\%)^{b}$ \\
\hline 1 & - & 20 & 1 & 33 \\
\hline 2 & - & 10 & 2 & 38 \\
\hline 3 & - & 5 & 4 & 57 \\
\hline 4 & 20 & 10 & 5 & 72 \\
\hline 5 & 20 & 50 & 2.2 & 33 \\
\hline 6 & 75 & 50 & 6.5 & 88 \\
\hline 7 & 75 & 100 & 5.2 & 85 \\
\hline 8 & 100 & 200 & 7 & 90 \\
\hline 9 & 250 & 100 & 8 & 96 \\
\hline
\end{tabular}

areaction conditions: POLITAG-L-F (96 mg), 1a (1 mmol), TMSCN (1.05 mmol), $60{ }^{\circ} \mathrm{C}$. ${ }^{b}$ conversion determined by $\mathrm{GC}$ analysis. 


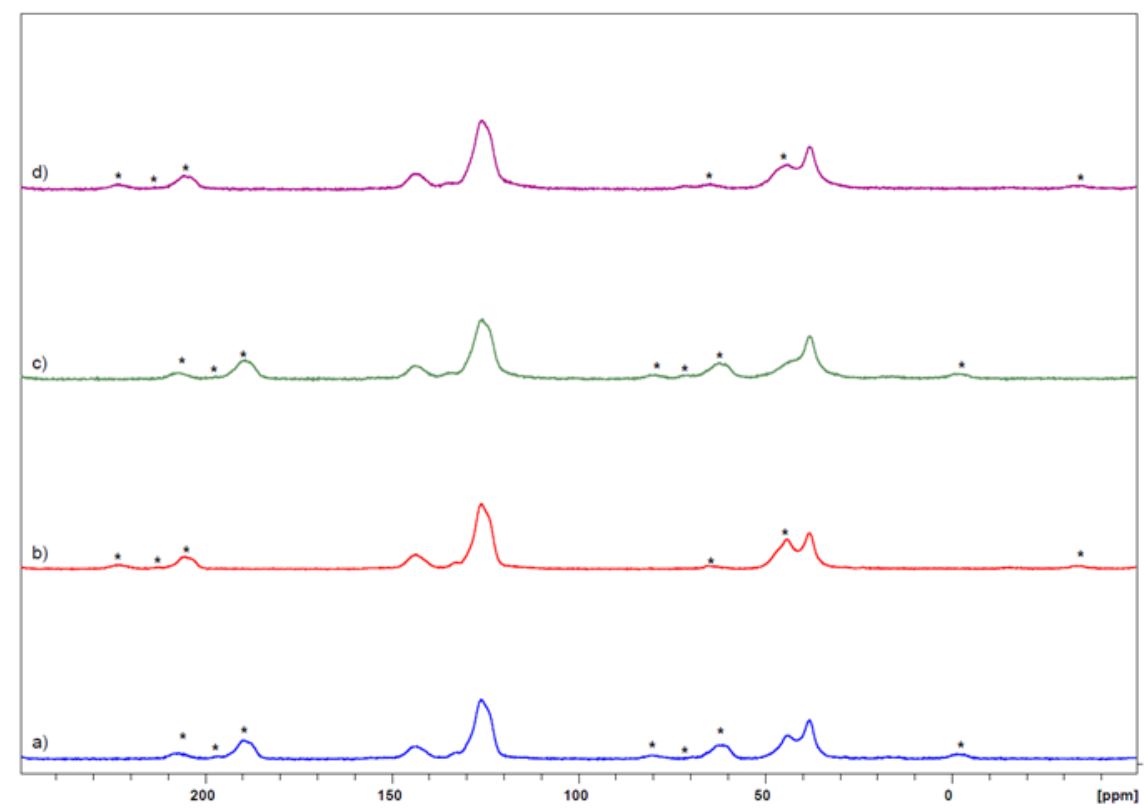

Figure S1. ${ }^{13} \mathrm{C}$ CP-MAS spectra of SP02-L-Cl at a) $8 \mathrm{KHz}$ and b) $10 \mathrm{KHz}$ and ${ }^{13} \mathrm{C}$ CP-MAS spectra of POLITAG-L-I at c) $8 \mathrm{KHz}$ and d) $10 \mathrm{KHz}$.

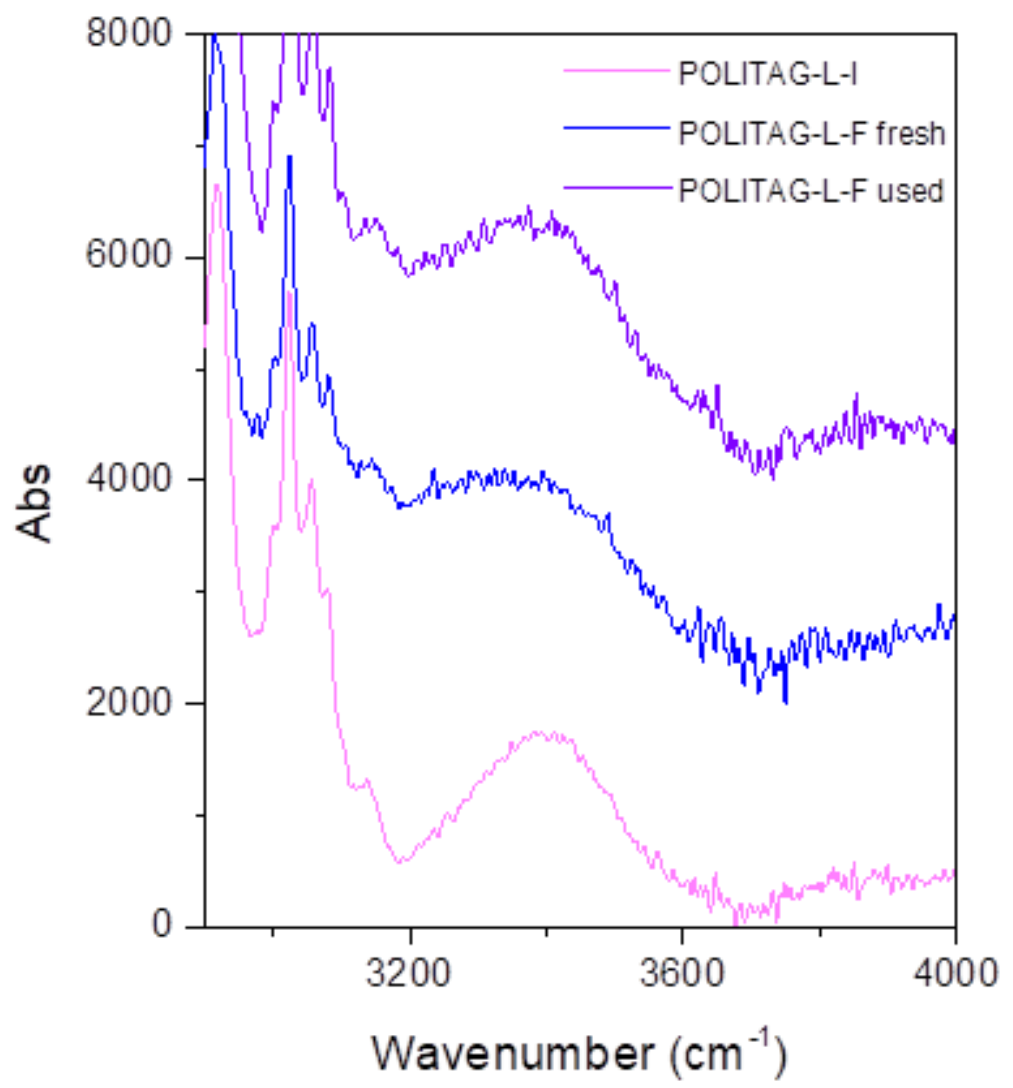

Figure S2. ATR-IR spectra of the OH-stretching region for the POLITAG-L-I and POLITAGs-L-F (fresh and used) 


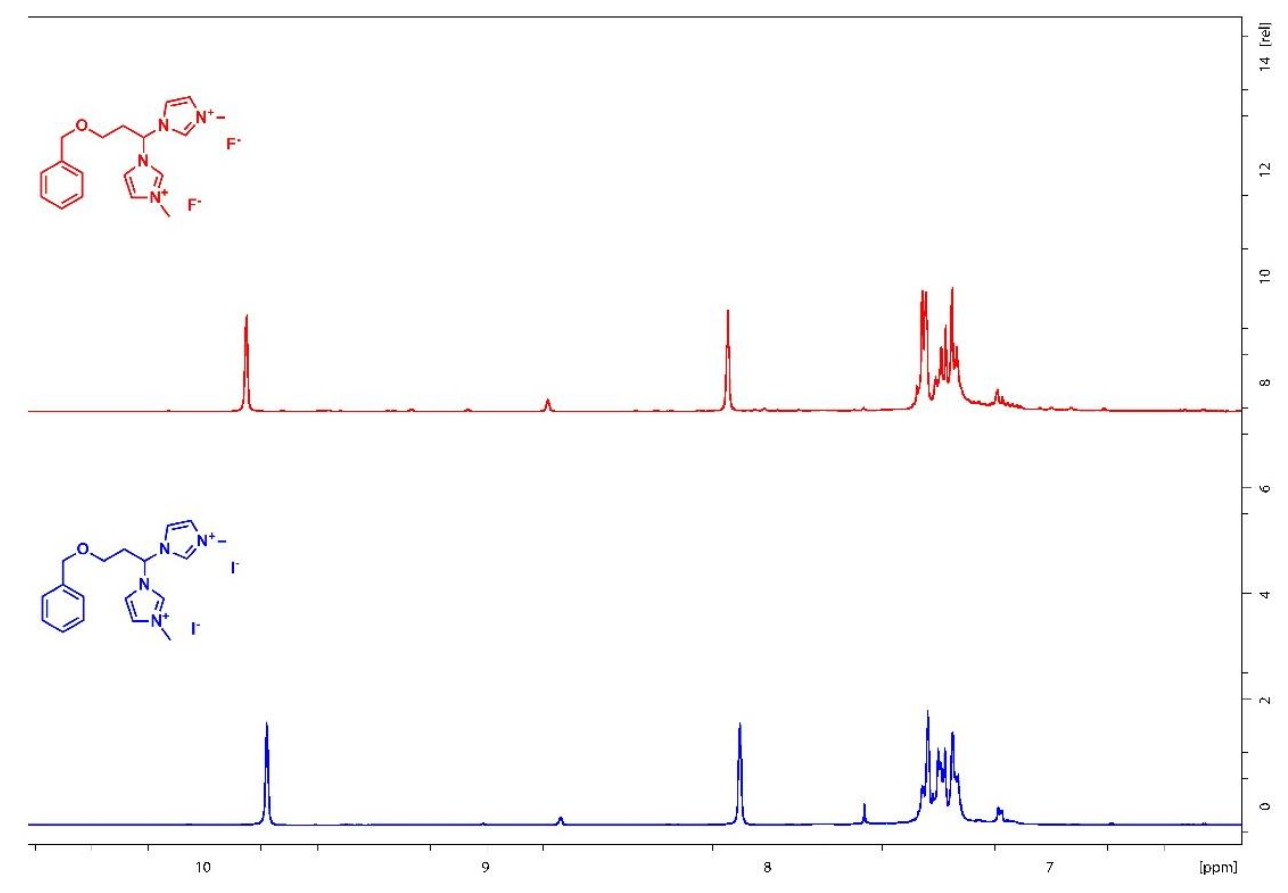

Figure S3. Comparison of $\mathrm{H}-\mathrm{NMR}$ shift in $\mathrm{CD}_{3} \mathrm{CN}$ of homogenous bis-imidazolium units with iodide (blue line) and fluoride (red line) counterion. Reaction conditions for homogeneous anion exchange: iodide-based ligand (132 mg) was placed in $10 \mathrm{~mL}$ aqueous solution of $\mathrm{KF}(10 \mathrm{M})$ and stirred at $60^{\circ} \mathrm{C}$. After $1 \mathrm{~h}$ the mixture was dried and the solid washed with $\mathrm{CH}_{3} \mathrm{CN}$. The organic phase was dried under reduced pressure to afford fluoride bis-imidazolium ionic-tag. 


\section{Data used for Environmental Index and Safety Hazard Index ${ }^{4}$}

\section{Substances parameters}

\begin{tabular}{llllllll}
\hline Substance & CAS & LD $_{50}$ & LC $_{50}$ & Log $\mathbf{k}_{\text {ow }}$ & $\begin{array}{l}\text { Henry law } \\
\text { constant }\end{array}$ & $\mathbf{C}_{\mathbf{w}}$ & $\mathbf{C}_{\mathbf{a}}$ \\
\hline Acetophenone & $98-86-2$ & 2081 & 2.13 & 1.61 & $1.04 \mathrm{E}-5$ & $3.33 \mathrm{E}-8$ & $1.41 \mathrm{E}-11$ \\
TMSCN $^{a}$ & $7677-24-9$ & 3.7 & 0.13 & 1.38 & $1.33 \mathrm{E}-4$ & $1.35 \mathrm{E}-8$ & $7.31 \mathrm{E}-11$ \\
\hline
\end{tabular}

aToxicity data refers to $\mathrm{HCN}$ where needed

Benign Index and Environmental Index parameters

\begin{tabular}{llllllll}
\hline Substance & Flash Point (K) & FP & CGP & CLP & RPP & OELP & $\boldsymbol{\Omega}(\mathrm{BI})$ \\
\hline Acetophenone & 355,15 & 0.810 & 0.244 & 1.081 & 0.750 & 10.020 & 12.906 \\
TMSCN $^{a}$ & 274,15 & 1.050 & 3.250 & 608.108 & 1.620 & 81,600 & 695.628 \\
\hline
\end{tabular}

Toxicity data refers to $\mathrm{HCN}$ where needed

Safety Hazard Index parameters

\begin{tabular}{lllll}
\hline Substance & BCP & INHTP & INGTP & $\Omega$ (SHI) \\
\hline Acetophenone & 0.140 & 3.03 & 25.07 & 28.24 \\
TMSCN $^{a}$ & 0.094 & 209.22 & 5715.62 & 5924.93 \\
\hline
\end{tabular}

a Toxicity data refers to $\mathrm{HCN}$ where needed

$\mathrm{BI}$ and SHI calculation over fractional weight for the imput materials

\begin{tabular}{cllllll}
\hline References $^{\mathbf{a}}$ & $\mathbf{f}_{\text {(acetophenone) }}$ & $\mathbf{f}_{(\mathrm{TMSCN})}$ & $\mathbf{E l}$ & $\mathbf{B I}^{\boldsymbol{b}}$ & SHZI & $\mathbf{S H I}^{\boldsymbol{c}}$ \\
\hline 40 & 0.502 & 0.498 & 0.497788 & 0.502212 & 0.497727 & 0.502273 \\
44 & 0.524 & 0.476 & 0.476846 & 0.523154 & 0.476199 & 0.523801 \\
57 & 0.251 & 0.749 & 0.73923 & 0.26077 & 0.745919 & 0.254081 \\
This work & 0.535 & 0.465 & 0.46568 & 0.53432 & 0.46472 & 0.53528 \\
\hline
\end{tabular}

areferences are related to the main text; ${ }^{\circ} \mathrm{BI}$ is defined as $1-\mathrm{El}, \mathrm{BI}=$ to 0 means low benign index while $B I=1$ means "green situation"; ' $\mathrm{SHI}$ is defined as 1-SHZI, SHI = to 0 means high risk for safety while SHI=1 means low risk for safety and therefore "green situation" 


\section{Radial polygon graphical representation for mass-related metrics}

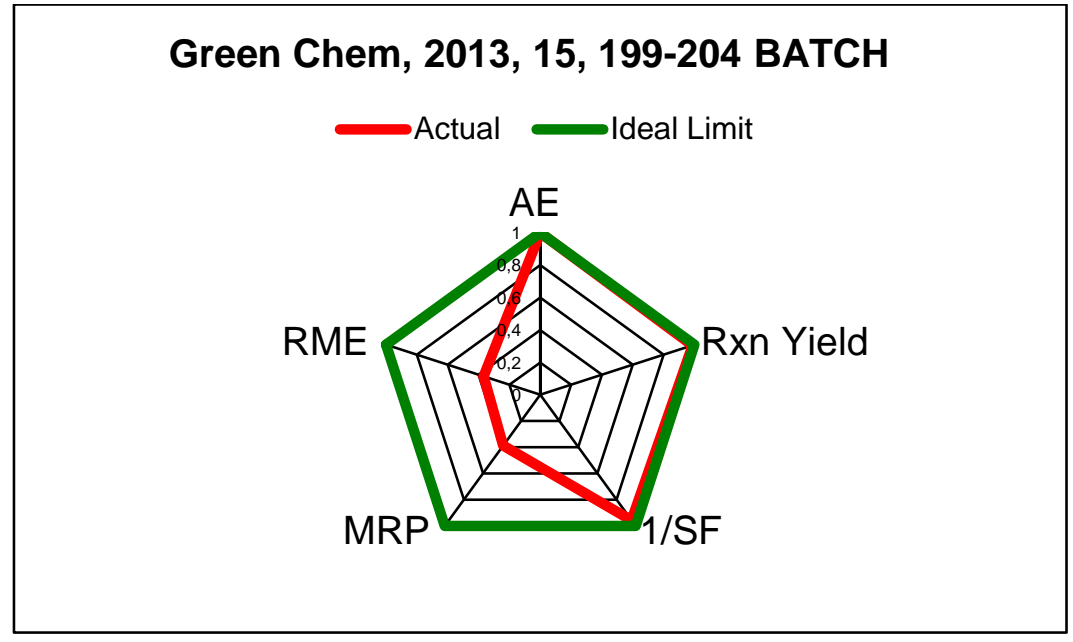

\begin{tabular}{|l|l|l|}
\hline parameter & actual & Ideal limit \\
\hline AE & 1,000 & 1 \\
\hline Rxn Yield & 0,988 & 1 \\
\hline 1/SF & 0,957 & 1 \\
\hline MRP & 0,388267209 & 1 \\
\hline RME & 0,367 & 1 \\
\hline & & \\
\hline VMR & 0,797185421 & \\
\hline
\end{tabular}

\section{Green Chem, 2013, 15, 199-204 FLOW}

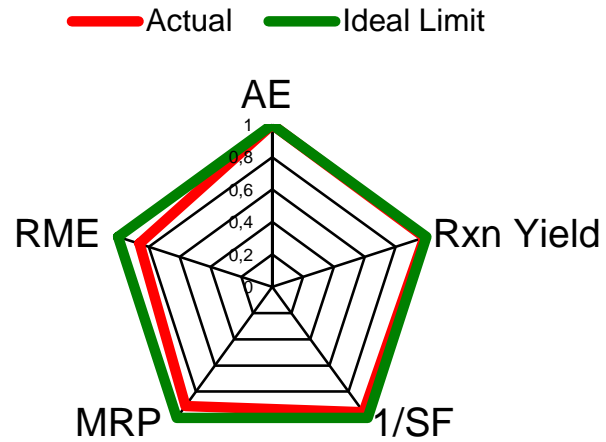

Green Chem 2014, 16, 1639-1647 FLOW

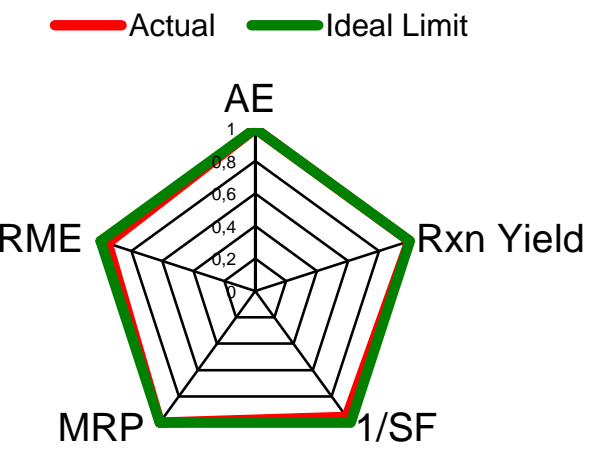

\begin{tabular}{|l|l|l|}
\hline parameter & actual & Ideal limit \\
\hline AE & 1,000 & 1 \\
\hline Rxn Yield & 0,998 & 1 \\
\hline $\mathbf{1 / S F}$ & 0,957 & 1 \\
\hline MRP & 1,000 & 1 \\
\hline RME & 0,955 & 1 \\
\hline & & \\
\hline VMR & 0,982324986 & \\
\hline
\end{tabular}




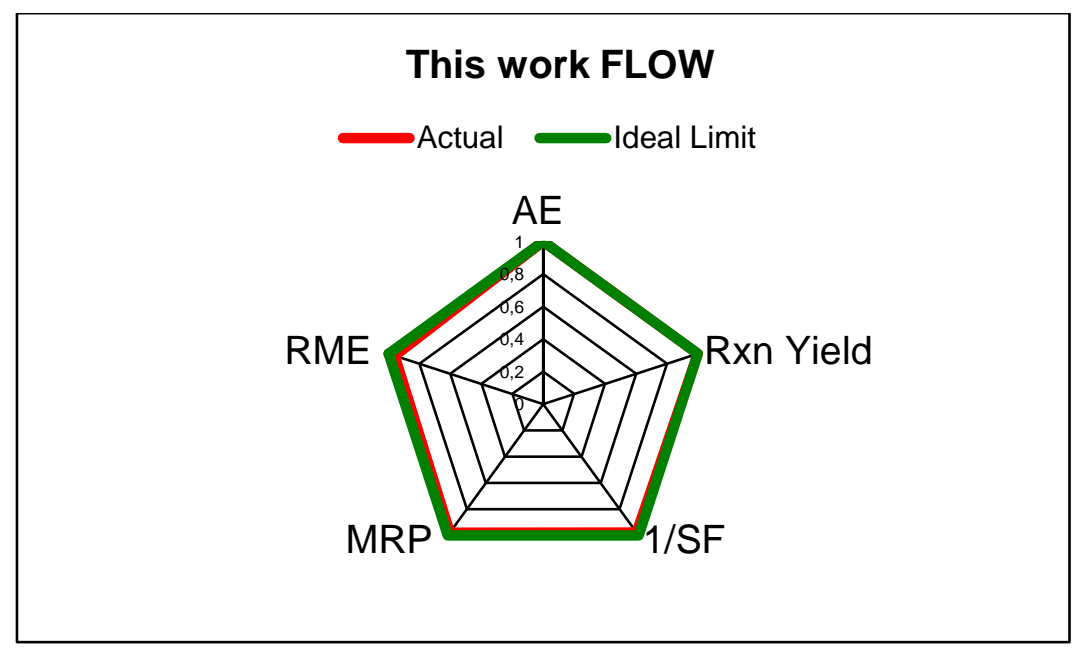

\begin{tabular}{|l|l|l|}
\hline parameter & actual & Ideal limit \\
\hline AE & 1,000 & 1 \\
\hline Rxn Yield & 1,000 & 1 \\
\hline 1/SF & 0,978 & 1 \\
\hline MRP & 0,979265321 & 1 \\
\hline RME & 0,961 & 1 \\
\hline & & \\
\hline VMR & 0,984429697 & \\
\hline
\end{tabular}

\section{References}

1. Mahmoudi, H.; Valentini, F.; Ferlin, F.; Bivona, L. A.; Anastasiou, I.; Fusaro, L.; Aprile, C.; Marrocchi, A.; Vaccaro. L. A tailored polymeric cationic tag-anionic Pd(II) complex as a catalyst for the lowleaching Heck-Mizoroki coupling in flow and in biomass-derived GVL. Green Chem., 2019, 21, 355360

2. Marrocchi, A.; Adriaensens, P.; Bartollini, E.; Barkakaty, B.; Carleer, R.; Chen, J.; Vaccaro, L. Novel cross-linked polystyrenes with large space network as tailor-made catalyst supports for sustainable media. Eur. Polym. J. 2015, 73, 391-401

3. Angelini, T.; Lanari, D.; Maggi, R.; Pizzo, F.; Sartori, G.; Vaccaro, L. Preparation and Use of Polystyryl$\mathrm{DABCOF}_{2}$ : An Efficient Recoverable and Reusable Catalyst for $\beta$-Azidation of $\alpha, \beta$-Unsaturated Ketones in Water. Adv. Synth. Catal. 2012, 354, 908 - 916.

4. The safety/hazard analysis have been conducted in accordance with the methods, calculations and equation reported by J. Andraos in Org. Process Res. Dev. 2013, 17, 175-192. Definitions and equation for multicompartment method (MCM) have been used in accordance with the data reported by J. Andraos in Org. Process Res. Dev. 2012, 16, 1482-1506. Log Kow reference: CRC Handbook of Chemistry and Physics, 91st ed., CRC Press: Boca Raton, 2009, Chapter 16, pp. 43-47 ARDP LD50 and LC50 and OEL, Flash point and risk phrases references and sources: MSDS files for each compound, Registry of Toxic Effects of Chemical Substances (RTECS) and NIOSH POCKET GUIDE TO CHEMICAL HAZARDS, National Institute for Occupational Safety and Health, September 2007, Publication No. 2005-149 (http://www.cdc.gov/niosh). QEissen factors: Eissen, M. Bewertung der Umweltverträglichkeit organisch-chemischer Synthesen. PhD thesis 2001, Universität Oldenburg, and Org. Process Res. Dev. 2013, 17, 175-192. 


\section{Characterization Data}

\begin{tabular}{|c|c|c|c|c|c|}
\hline Chem. Name & \multicolumn{5}{|c|}{ 2-phenyl-2-((trimethylsilyl)oxy)propanenitrile (2a) } \\
\hline \multirow[t]{2}{*}{ Lit. Ref. } & \multicolumn{5}{|c|}{$\begin{array}{l}\text { G. Strappaveccia, D. Lanari, D. Gelman, F. Pizzo, O. Rosati, M. Curini, L. } \\
\text { Vaccaro. Green Chem., 2013, 15, 199-204. }\end{array}$} \\
\hline & $1 a$ & \multicolumn{2}{|c|}{$\begin{array}{l}\text { SolFC } \\
60^{\circ} \mathrm{C}, 1 \mathrm{~h}\end{array}$} & OTMs & \\
\hline \multicolumn{6}{|c|}{$\begin{array}{l}\text { METHOD: } \\
\text { In a screw capped vial equipped with a magnetic stirrer POLITAG-L-F }(8.06 \mathrm{mg}, 2 \mathrm{~mol} \%, 0.62 \\
\mathrm{mmol} / \mathrm{g}) \text {, acetophenone }(\mathbf{1 a})(58 \mu \mathrm{L}, 0.5 \mathrm{mmol}) \text {, e TMSCN }(66 \mu \mathrm{L}, 1.05 \mathrm{eq}) \text { were consecutively } \\
\text { added and the resulting mixture was left under stirring at } 60^{\circ} \mathrm{C} \text { for } 1 \mathrm{~h} \text {. EtOAc }(1 \mathrm{~mL}) \text { was used } \\
\text { for recover the product and removed under reduced pressure to afford } 109 \mathrm{mg} \text { of } 2 \text {-phenyl-2- } \\
((\text { trimethylsilyl)oxy)propanenitrile }(\mathbf{2 a}) \text { as an oil }(99 \% \text { yield }) . \\
\text { E-Factor }(\text { batch })=[60.0 \mathrm{mg}(\mathbf{1 a})+52.3 \mathrm{mg}(\mathrm{TMSCN})+900 \mathrm{mg}(\text { EtOAc-wokup })-109 \mathrm{mg}(\mathbf{2 a})] / \\
109 \mathrm{mg}(\mathbf{2 a})=8.3\end{array}$} \\
\hline Mol Formula & & $\mathrm{H}_{17} \mathrm{NOS}$ & & m.p. & Oil \\
\hline \multirow{5}{*}{$\begin{array}{c}{ }^{1} \mathrm{H} \mathrm{NMR} \\
400 \mathrm{MHz} \\
\mathrm{CDCl}_{3}\end{array}$} & $\delta$ value & No. $\mathrm{H}$ & Mult. & j value/Hz & \\
\hline & 7.55 & 2 & $d$ & 8 & \\
\hline & $7.35-7.42$ & 3 & $m$ & & \\
\hline & 1.86 & 3 & $s$ & & \\
\hline & 0.18 & 9 & $s$ & & \\
\hline \multicolumn{6}{|c|}{${ }^{13} \mathrm{C}$ NMR (100.6 MHz, $\left.\mathrm{CDCl}_{3}\right) \delta: 142.1 ; 128.8 ; 124.8 ; 121.8 ; 71.8 ; 33.7 ; 1.2}$. \\
\hline \multicolumn{6}{|c|}{$\begin{array}{l}\text { GC-EIMS (m/z, \%): } 219\left(\mathrm{M}^{+},<1\right) ; 205(15) ; 204(78) ; 178(18) ; 177(100) ; 135(23) ; 105(66) ; \\
103 \text { (20); } 84(18) ; 77(36) ; 76(15) ; 75(78) ; 73(18) ; 51(15) .\end{array}$} \\
\hline
\end{tabular}




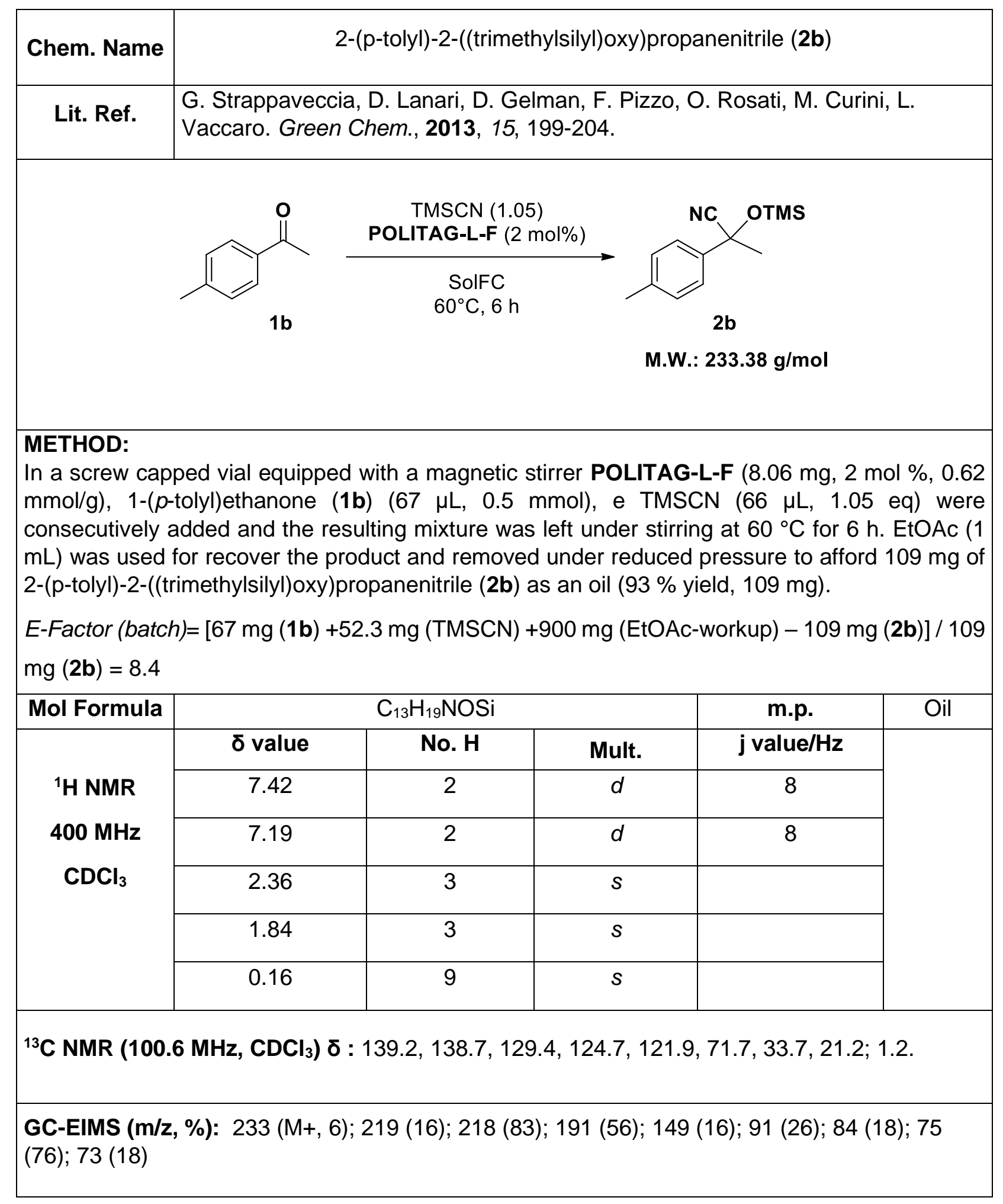




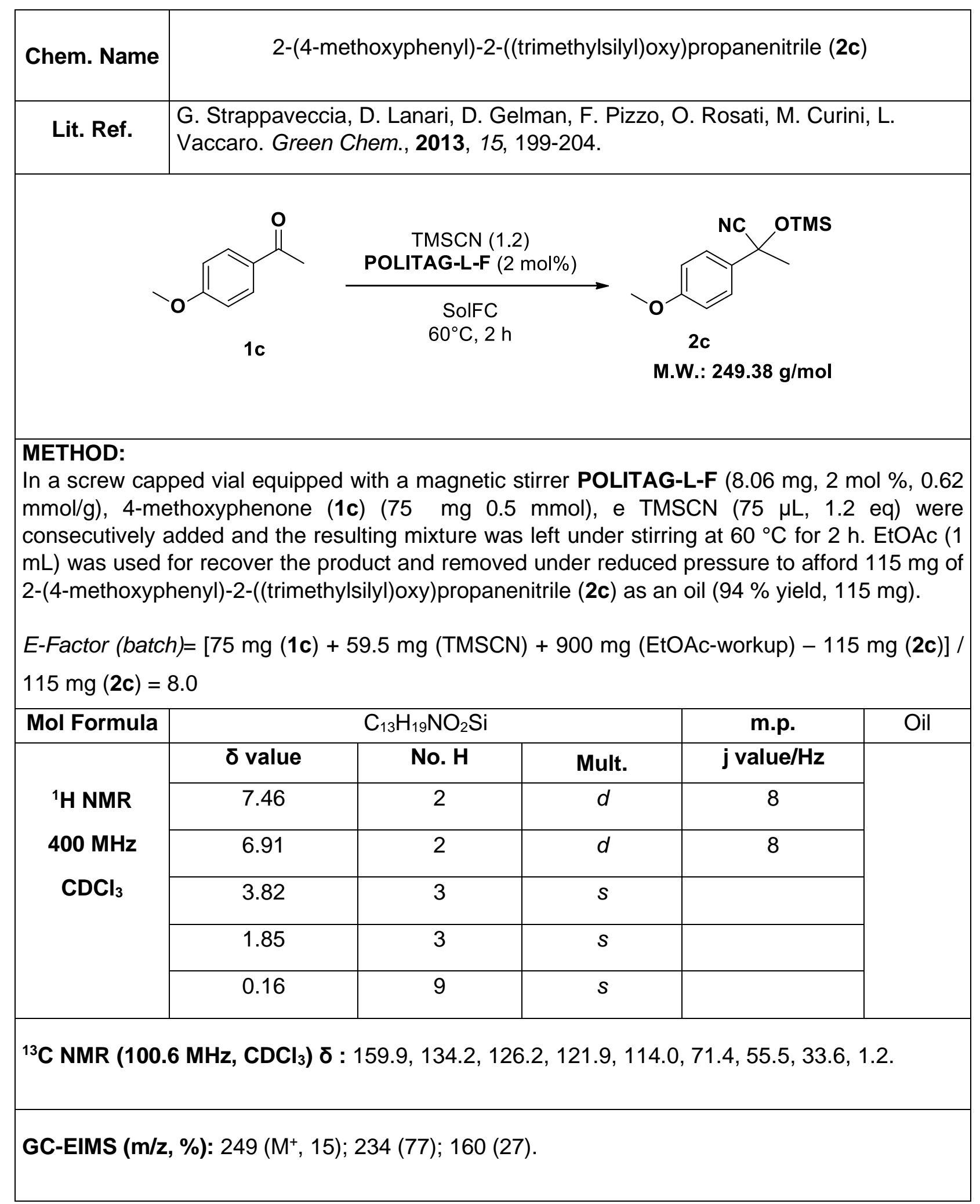




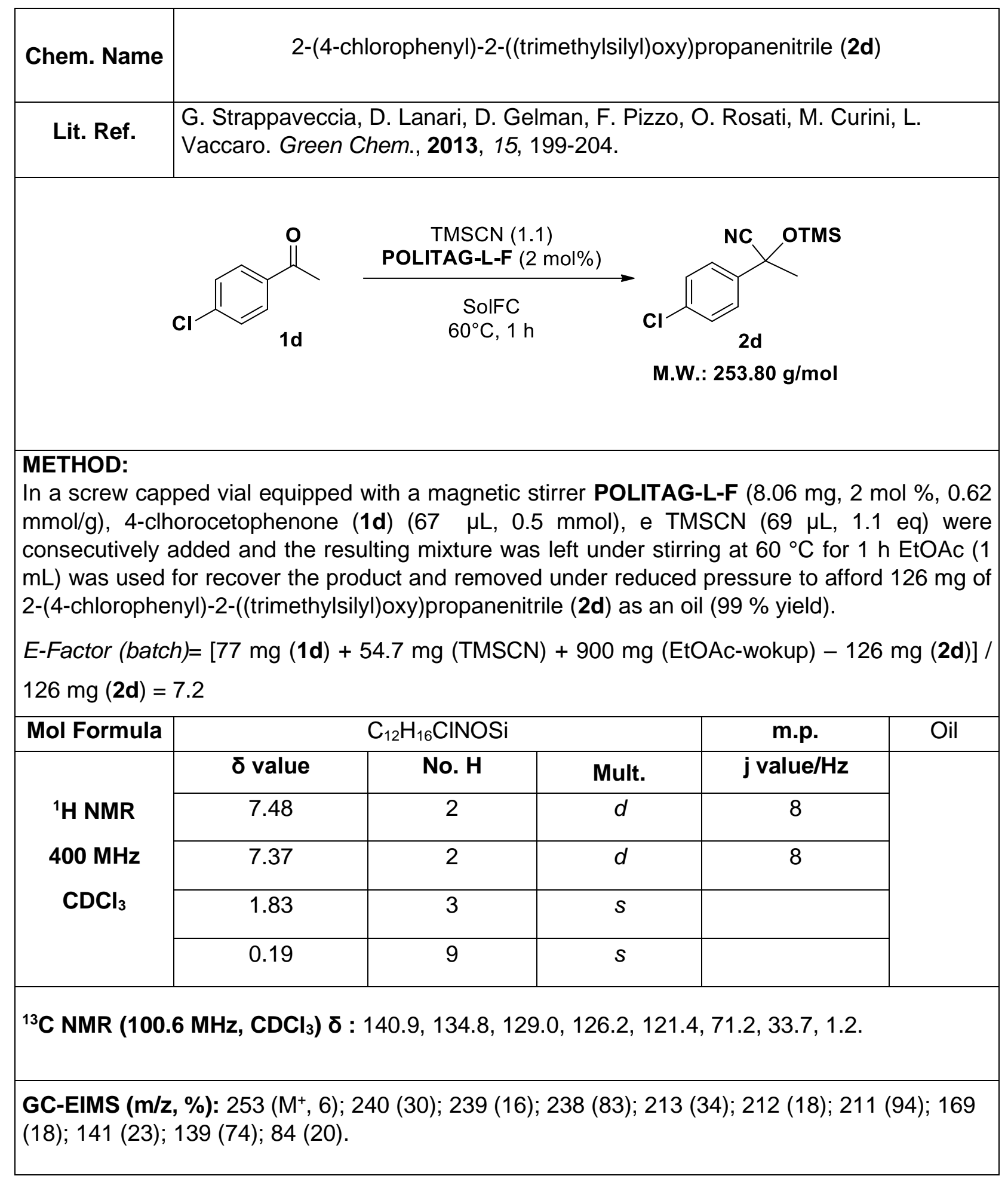




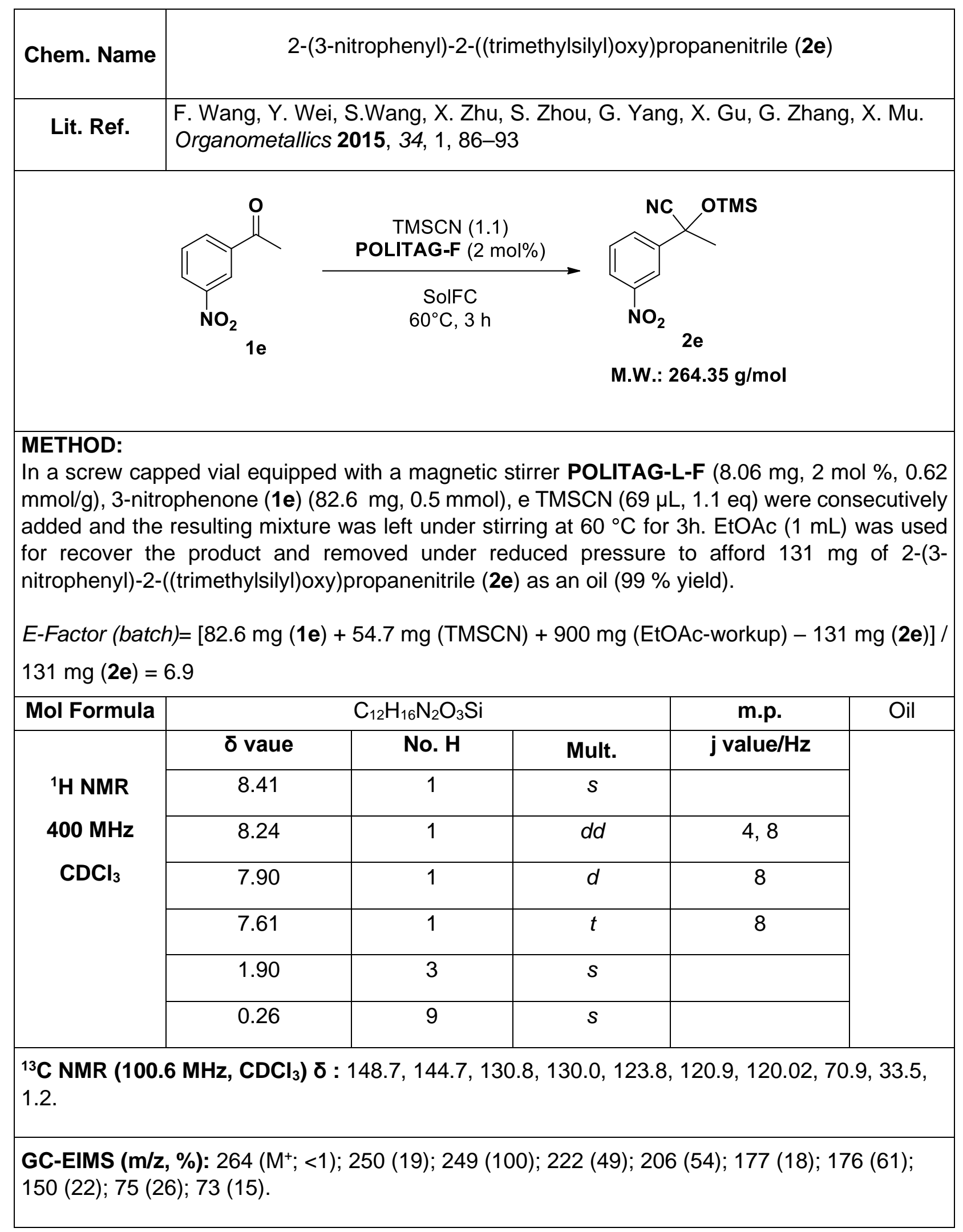




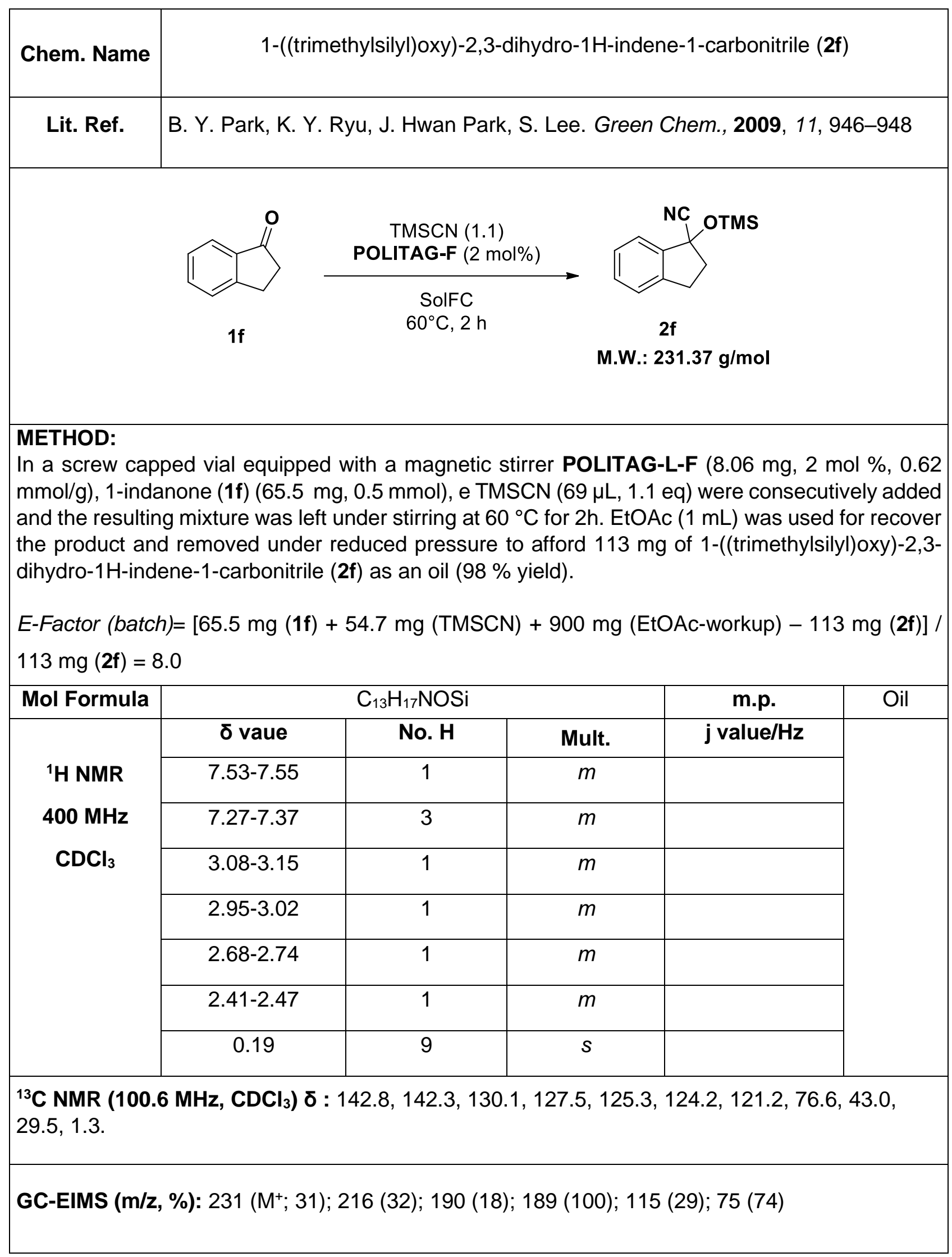




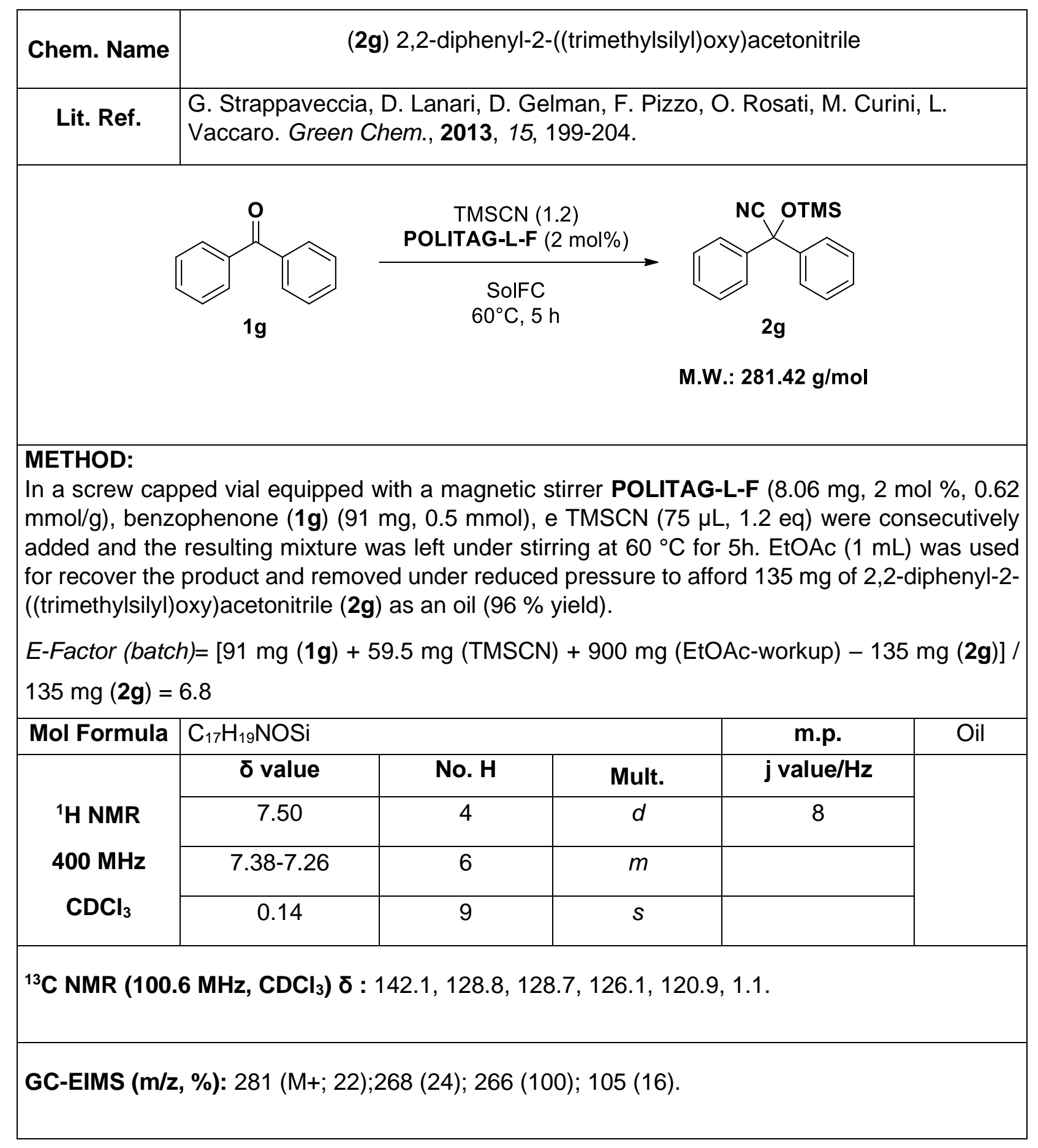




\section{Chem. Name \\ 2-(pyridin-2-yl)-2-((trimethylsilyl)oxy)propanenitrile (2h)}

Lit. Ref.

G. Strappaveccia, D. Lanari, D. Gelman, F. Pizzo, O. Rosati, M. Curini, L. Vaccaro. Green Chem., 2013, 15, 199-204.<smiles>CC(=O)c1ccccn1</smiles>

$1 \mathrm{~h}$

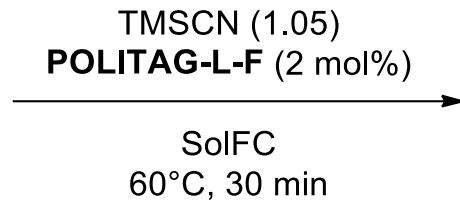

$60^{\circ} \mathrm{C}, 30 \mathrm{~min}$<smiles>COC(C)(C#N)c1ccccn1</smiles>

$2 \mathrm{~h}$

M.W.: $220.34 \mathrm{~g} / \mathrm{mol}$

\section{METHOD:}

In a screw capped vial equipped with a magnetic stirrer POLITAG-L-F (8.06 mg, 2 mol \%, 0.62 $\mathrm{mmol} / \mathrm{g}$ ), 1-(pyridin-2-yl)ethanone (1h) $(56 \mu \mathrm{L}, 0.5 \mathrm{mmol}$ ), e TMSCN (66 $\mu \mathrm{L}, 1.05 \mathrm{eq})$ were consecutively added and the resulting mixture was left under stirring at $60^{\circ} \mathrm{C}$ for $30 \mathrm{~min}$. EtOAc (1 $\mathrm{mL}$ ) was used for recover the product and removed under reduced pressure to afford $104 \mathrm{mg}$ of 2-(pyridin-2-yl)-2-((trimethylsilyl)oxy)propanenitrile (2h) as an oil (94\% yield).

E-Factor (batch) $=[60.5 \mathrm{mg}(\mathbf{1 h})+52.3 \mathrm{mg}(\mathrm{TMSCN})+900 \mathrm{mg}($ EtOAc-workup) $-104 \mathrm{mg}(\mathbf{2 h})] /$ $104 \mathrm{mg}(\mathbf{2 h})=8.7$

\begin{tabular}{|c|c|c|c|c|c|}
\hline Mol Formula & \multicolumn{3}{|c|}{$\mathrm{C}_{11} \mathrm{H}_{16} \mathrm{~N}_{2} \mathrm{OSi}$} & m.p. & Oil \\
\hline \multirow{7}{*}{$\begin{array}{c}{ }^{1} \mathrm{H} \mathrm{NMR} \\
400 \mathrm{MHz} \\
\mathrm{CDCl}_{3}\end{array}$} & $\delta$ value & No. $\mathrm{H}$ & Mult. & j value $/ \mathrm{Hz}$ & \\
\hline & 8.61 & 1 & $d$ & 4 & \\
\hline & 7.75 & 1 & $t$ & 8 & \\
\hline & 7.58 & 1 & $d$ & 8 & \\
\hline & $7.25-7.28$ & 1 & $m$ & & \\
\hline & 1.91 & 3 & $s$ & & \\
\hline & 0.24 & 9 & $s$ & & \\
\hline
\end{tabular}

${ }^{13} \mathrm{C}$ NMR (100.6 MHz, $\left.\mathrm{CDCl}_{3}\right) \delta: 160.2,149.2,137.4,123.6,121.5,119.1,73.0,31.4,1.3$.

GC-EIMS (m/z, \%): 220 (M+, 18); 205 (100); 190 (17); 179 (19); 178 (62); 78 (20); 75 (18). 


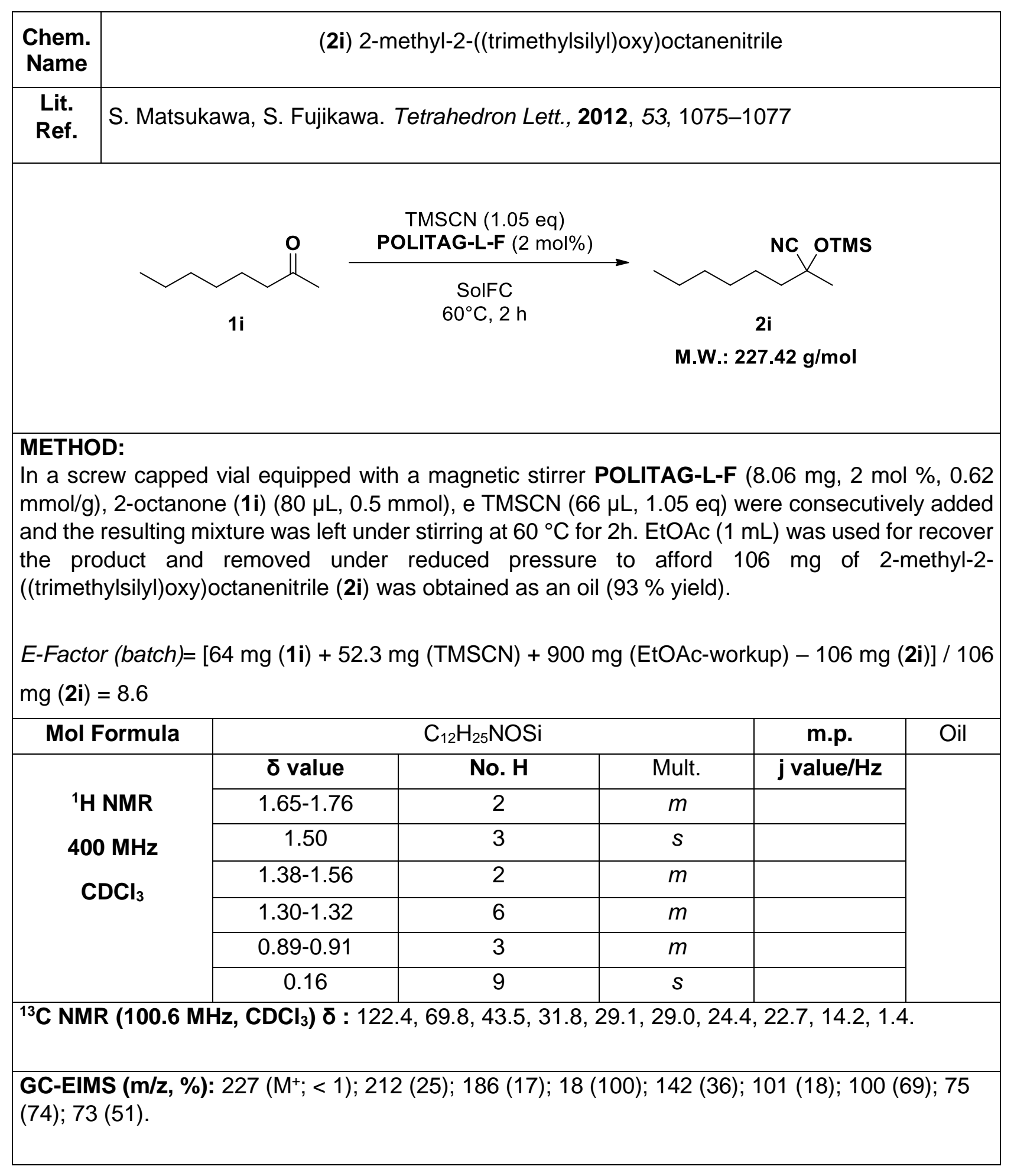




\begin{tabular}{|c|c|c|c|c|c|}
\hline Chem. Name & \multicolumn{5}{|c|}{ 1-((trimethylsilyl)oxy)cyclohexanecarbonitrile $(\mathbf{2} \mathbf{j})$} \\
\hline \multirow[t]{2}{*}{ Lit. Ref. } & \multicolumn{5}{|c|}{$\begin{array}{l}\text { S. Martín, R. Porcar, E. Peris, M. I. Burguete, E. García-Verdugo, S. V. Luis. } \\
\text { Green Chem., 2014, 16, 1639-1647 }\end{array}$} \\
\hline & $1 \mathrm{j}$ & $\begin{array}{c}\text { ISCN }(1 \\
\text { TAG-L-F } \\
\text { SolF } \\
60^{\circ} \mathrm{C},\end{array}$ & $\%)$ & $5 \mathrm{~g} / \mathrm{mol}$ & 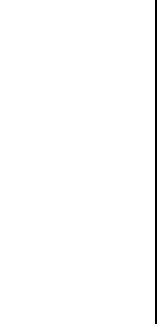 \\
\hline \multicolumn{6}{|c|}{ 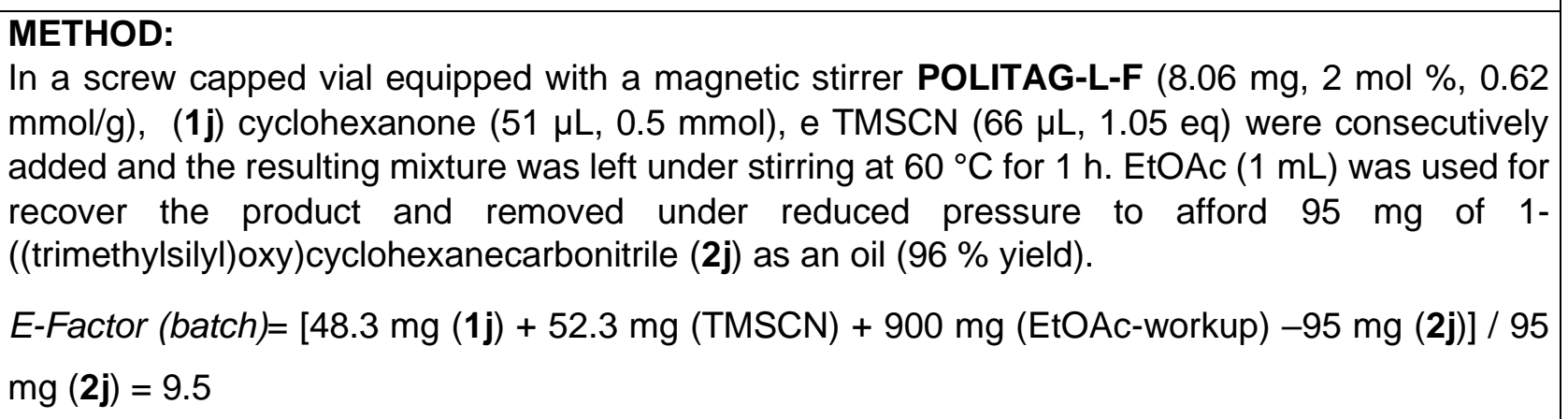 } \\
\hline \multicolumn{2}{|c|}{ Mol Formula } & \multicolumn{2}{|c|}{$\mathrm{C}_{10} \mathrm{H}_{19} \mathrm{NOSi}$} & m.p. & Oil \\
\hline \multirow{3}{*}{$\begin{array}{c}{ }^{1} \mathrm{H} \mathrm{NMR} \\
400 \mathrm{MHz} \\
\mathrm{CDCl}_{3}\end{array}$} & $\delta$ value & No. $\mathrm{H}$ & Mult. & j value/Hz & \\
\hline & $1.53-1.78$ & 10 & $m$ & & \\
\hline & 0.23 & 9 & $s$ & & \\
\hline \multicolumn{6}{|c|}{${ }^{13} \mathrm{C}$ NMR (100.6 MHz, $\left.\mathrm{CDCl}_{3}\right) \delta: 122.1,38.5,38.0,24.7,24.6,22.8,22.6,1.6}$. \\
\hline GC-EIMS (m/z, & )$: 197\left(\mathrm{M}^{+},<\right.$ & $6(15)$ & $00) ; 75$ & & \\
\hline
\end{tabular}




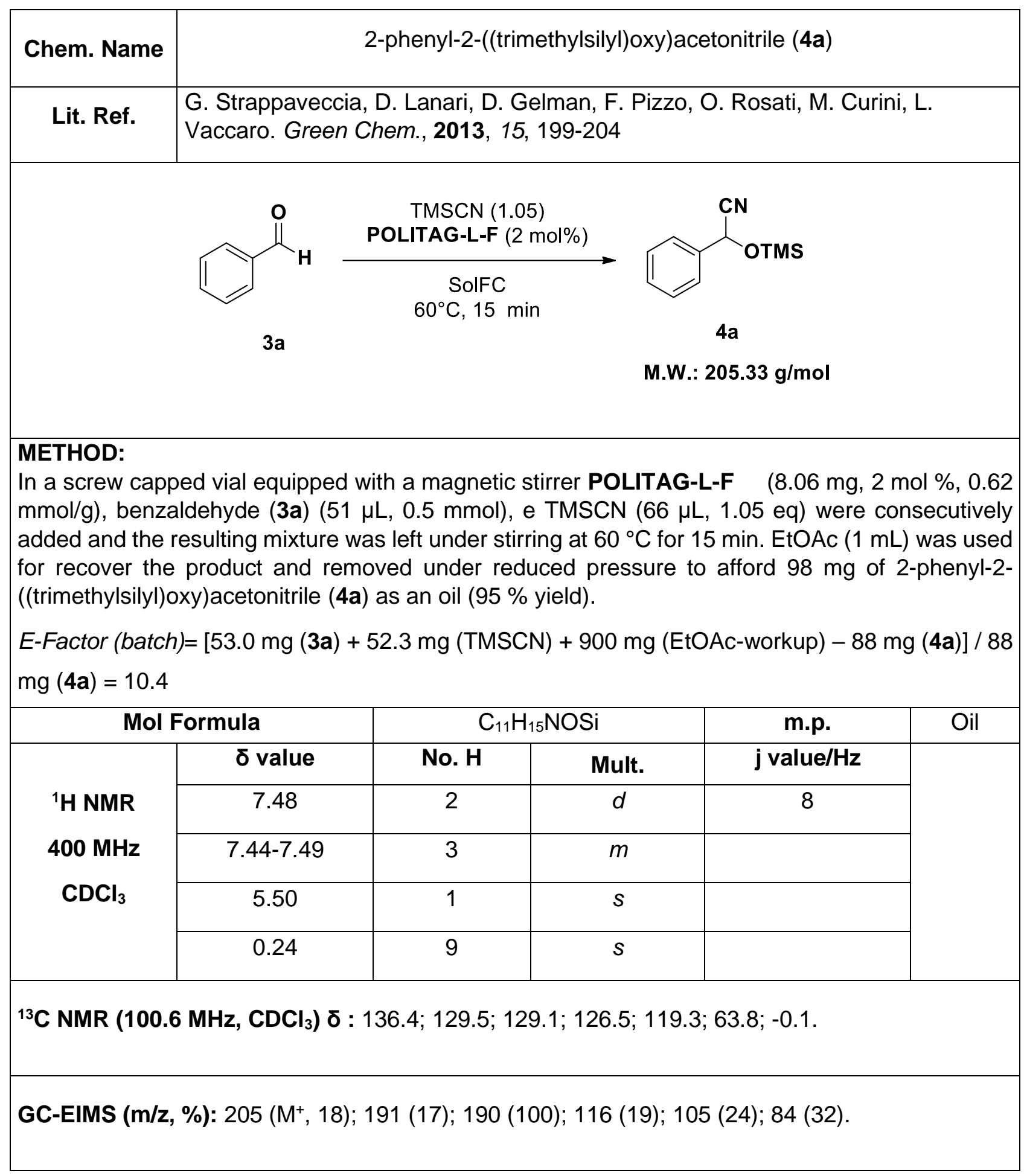




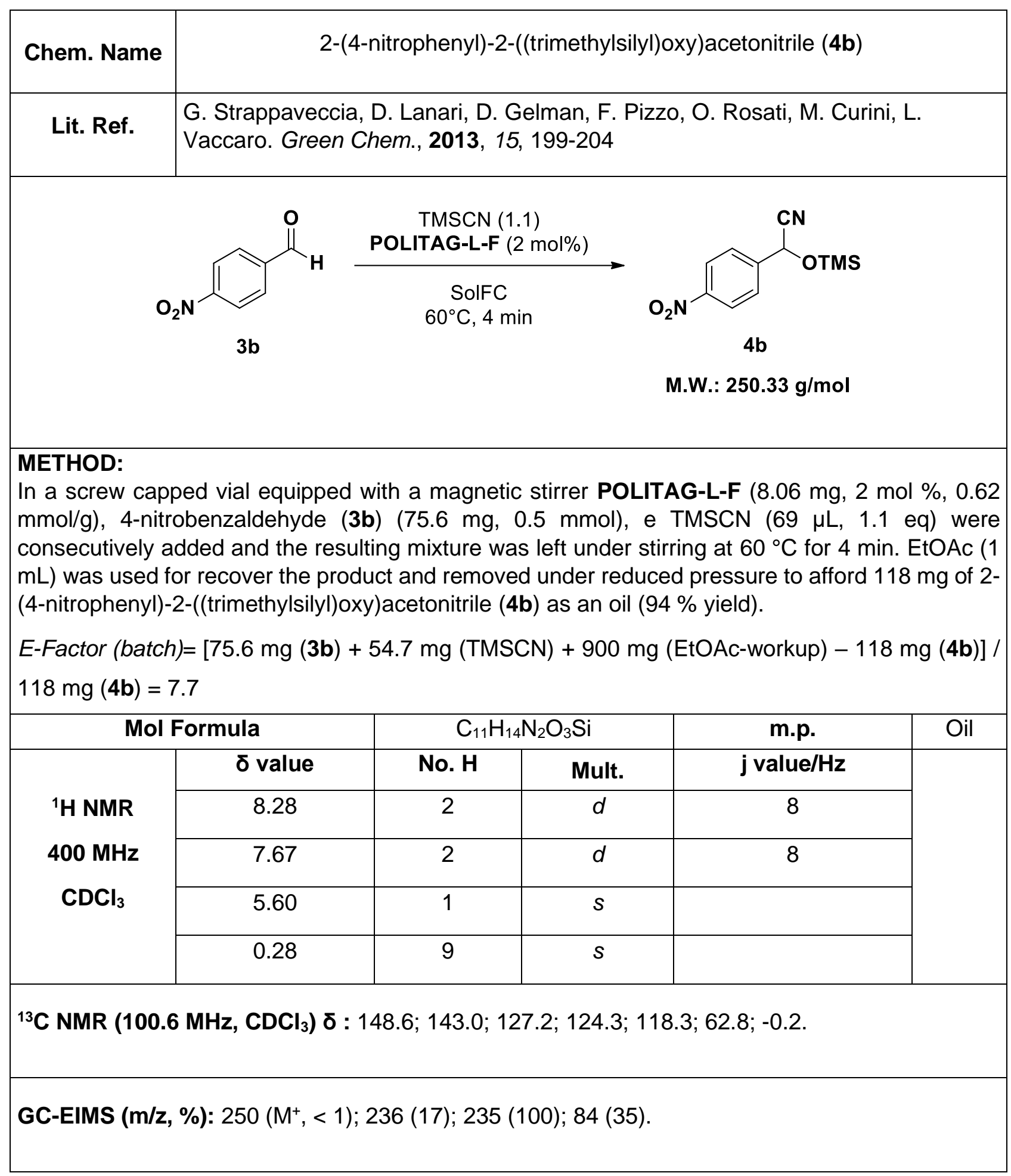




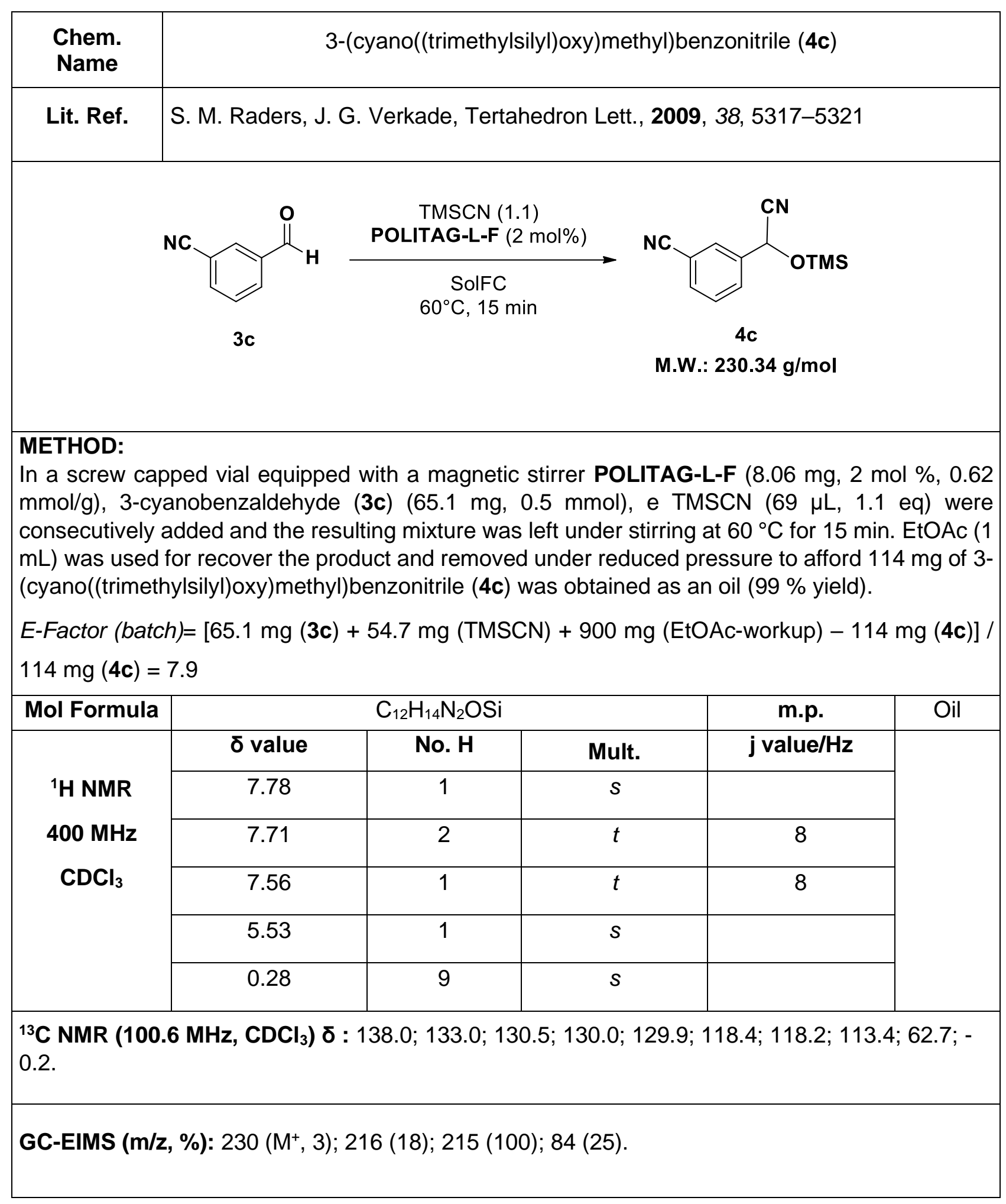




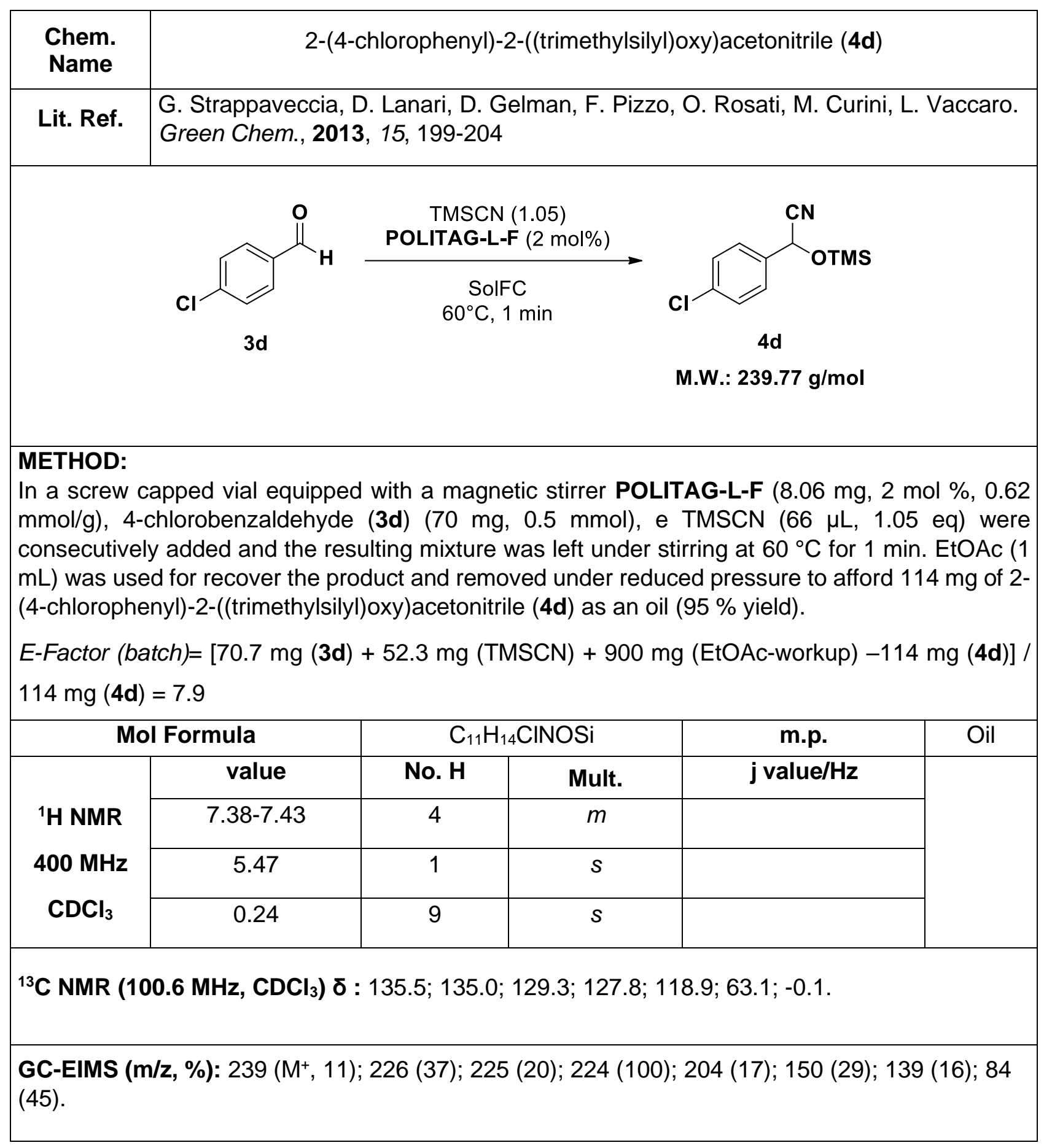




\begin{tabular}{|c|c|c|c|c|c|}
\hline Chem. Name & \multicolumn{5}{|c|}{ 2-(3-chlorophenyl)-2-((trimethylsilyl)oxy)acetonitrile (4e) } \\
\hline Lit. Ref. & \multicolumn{5}{|c|}{$\begin{array}{l}\text { G. Strappaveccia, D. Lanari, D. Gelman, F. Pizzo, O. Rosati, M. Curini, L. } \\
\text { Vaccaro. Green Chem., 2013, 15, 199-204 }\end{array}$} \\
\hline \multicolumn{2}{|c|}{$\underbrace{c 1}_{3 e}$} & $\begin{array}{r}\text { TMSCN } \\
\text { POLITAG-L-F } \\
\text { SolF } \\
60^{\circ} \mathrm{C}, 1\end{array}$ & $\longrightarrow$ & $\int_{4 e}^{C N}$ OTMS & \\
\hline \multicolumn{6}{|c|}{ 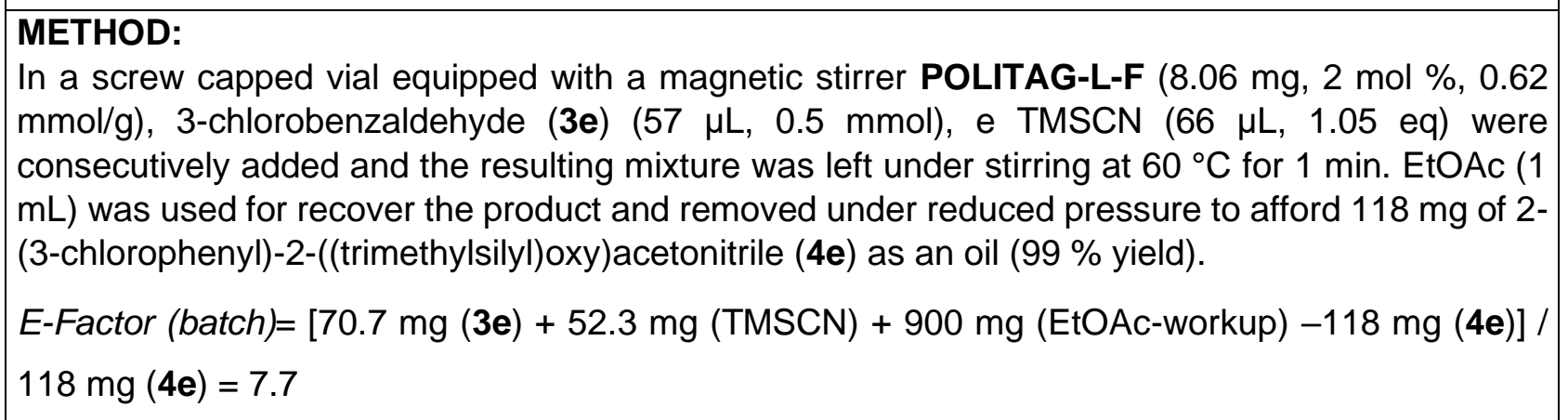 } \\
\hline \multicolumn{2}{|c|}{ Mol Formula } & \multicolumn{2}{|c|}{$\mathrm{C}_{11} \mathrm{H}_{14} \mathrm{CINOSi}$} & m.p. & Oil \\
\hline \multirow{5}{*}{$\begin{array}{c}{ }^{1} \mathrm{H} \text { NMR } \\
400 \mathrm{MHz} \\
\mathrm{CDCl}_{3}\end{array}$} & $\delta$ value & No. $\mathrm{H}$ & Mult. & j value/Hz & \\
\hline & 7.47 & 1 & $s$ & & \\
\hline & $7.26-7.36$ & 3 & $m$ & & \\
\hline & 5.47 & 1 & $s$ & & \\
\hline & 0.26 & 9 & $s$ & & \\
\hline \multicolumn{6}{|c|}{ 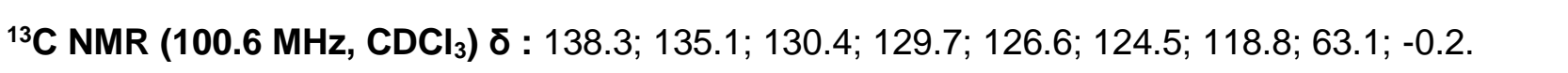 } \\
\hline GC-EIMS (m/z & $\%): 240\left(\mathrm{M}^{+}, 2\right)$ & $226(38) ; 225$ & $4(100)$ & & \\
\hline
\end{tabular}




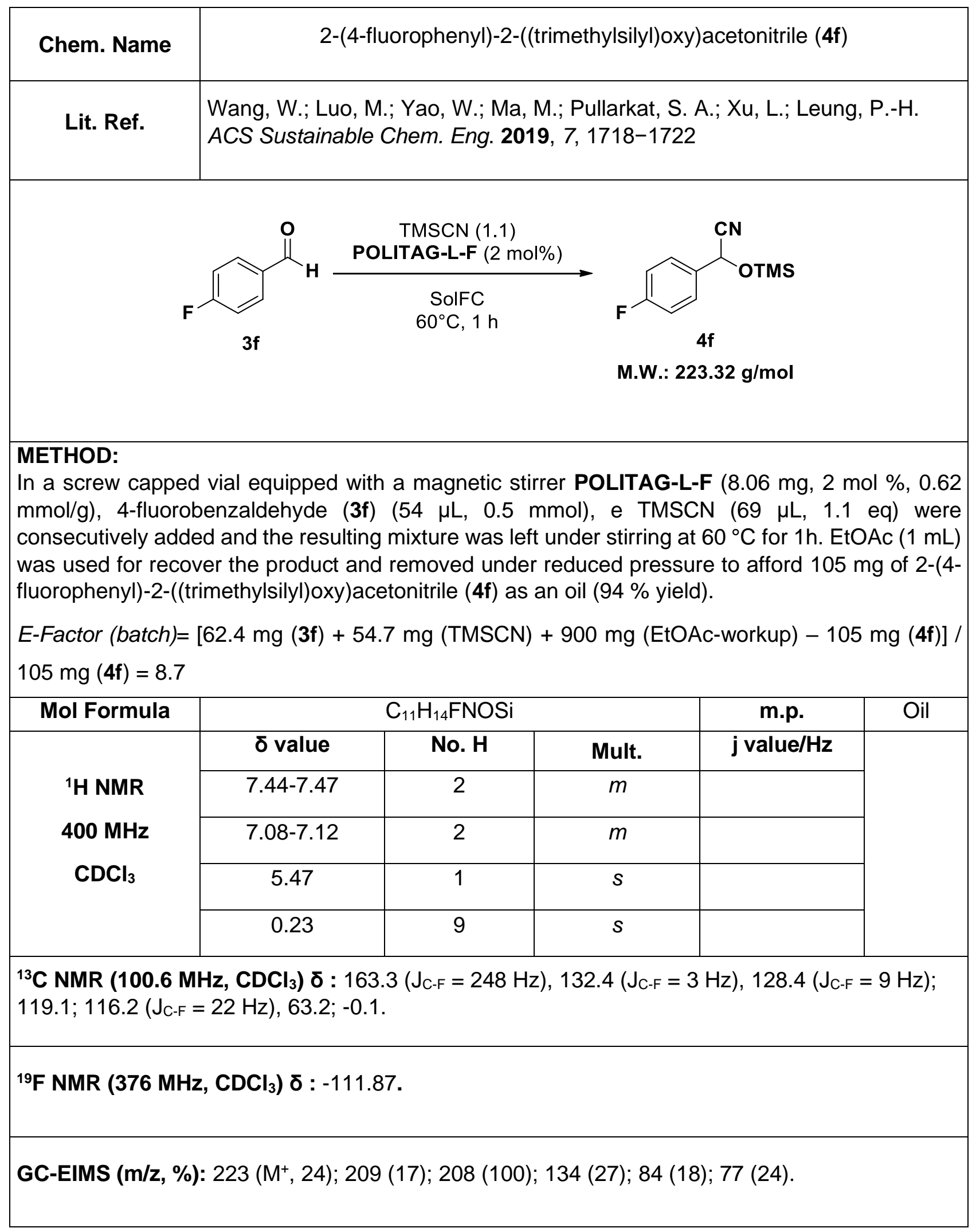




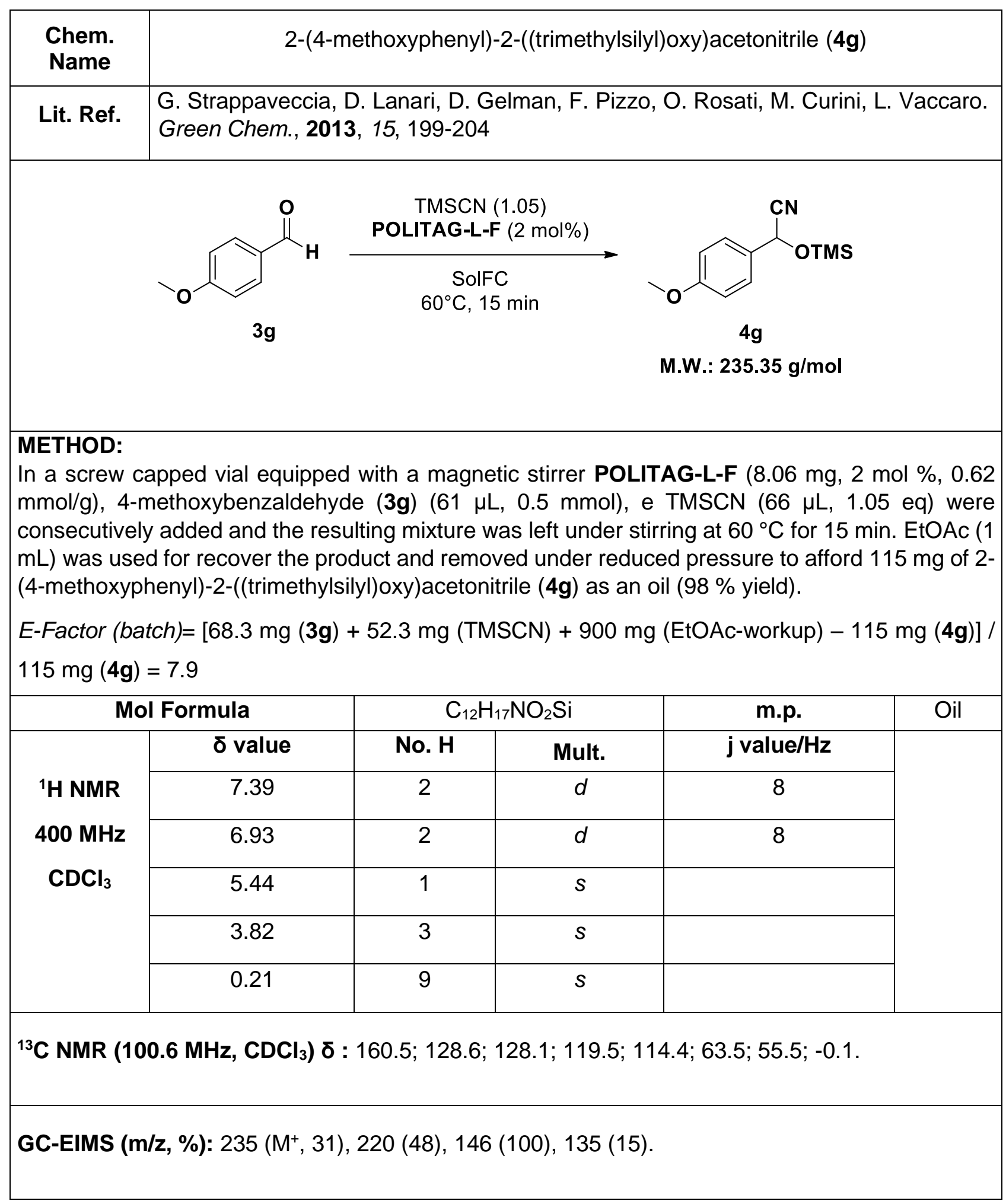




\begin{tabular}{|c|c|}
\hline Chem. Name & 2-(4-isobutylphenyl)-2-((trimethylsilyl)oxy)acetonitrile (4h) \\
\hline Lit. Ref. & \\
\hline
\end{tabular}

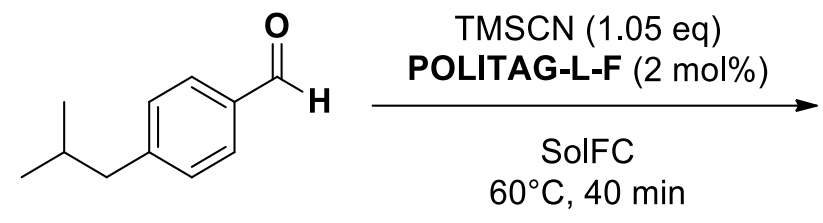

$3 \mathrm{~h}$<smiles>COC(C#N)c1ccc(CC(C)C)cc1</smiles>

4h

M.W.: $261.43 \mathrm{~g} / \mathrm{mol}$

\section{METHOD:}

In a screw capped vial equipped with a magnetic stirrer POLITAG-L-F $(8.06 \mathrm{mg}, 2 \mathrm{~mol} \%, 0.62$ $\mathrm{mmol} / \mathrm{g})$, 4-isobutylbenzaldehyde (3h) $(81 \mu \mathrm{L}, 0.5 \mathrm{mmol})$, e TMSCN (66 $\mu \mathrm{L}, 1.05 \mathrm{eq})$ were consecutively added and the resulting mixture was left under stirring at $60^{\circ} \mathrm{C}$ for $40 \mathrm{~min}$. EtOAc $(1$ $\mathrm{mL}$ ) was used for recover the product and removed under reduced pressure to afford $123 \mathrm{mg}$ of 2 (4-isobutylphenyl)-2-((trimethylsilyl)oxy)acetonitrile (4h) as an oil (94\% yield).

E-Factor $($ batch $)=[81.0 \mathrm{mg}(\mathbf{3 h})+54.7 \mathrm{mg}(\mathrm{TMSCN})+900 \mathrm{mg}($ EtOAc-workup $)-123 \mathrm{mg}(\mathbf{4 h})] /$ $123 \mathrm{mg}(\mathbf{4 h})=7.4$

\begin{tabular}{|c|c|c|c|c|c|}
\hline Mol Formula & \multicolumn{3}{|c|}{$\mathrm{C}_{15} \mathrm{H}_{23} \mathrm{NOSi}$} & m.p. & Oil \\
\hline \multirow{8}{*}{$\begin{array}{c}{ }^{1} \mathrm{H} \mathrm{NMR} \\
400 \mathrm{MHz} \\
\mathrm{CDCl}_{3}\end{array}$} & $\delta$ value & No. $\mathrm{H}$ & Mult. & j value/Hz & \\
\hline & 7.37 & 2 & $d$ & 8 & \\
\hline & 7.19 & 2 & $d$ & 8 & \\
\hline & 5.47 & 1 & $s$ & & \\
\hline & 2.49 & 2 & $d$ & 8 & \\
\hline & $1.83-1.90$ & 1 & $m$ & & \\
\hline & 0.91 & 6 & $d$ & 4 & \\
\hline & 0.22 & 9 & $s$ & & \\
\hline
\end{tabular}

${ }^{13} \mathrm{C}$ NMR (100.6 MHz, $\left.\mathrm{CDCl}_{3}\right) \delta: 143.3,133.7,129.8,126.4,119.5,63.8,45.2,30.3,22.4,-0.1$.

GC-EIMS (m/z, \%): 261 (M+, 17); 247 (22); 246 (100); 130 (20); 129 (22); 105 (28); 91 (16); 75 (26); 73 (35). 


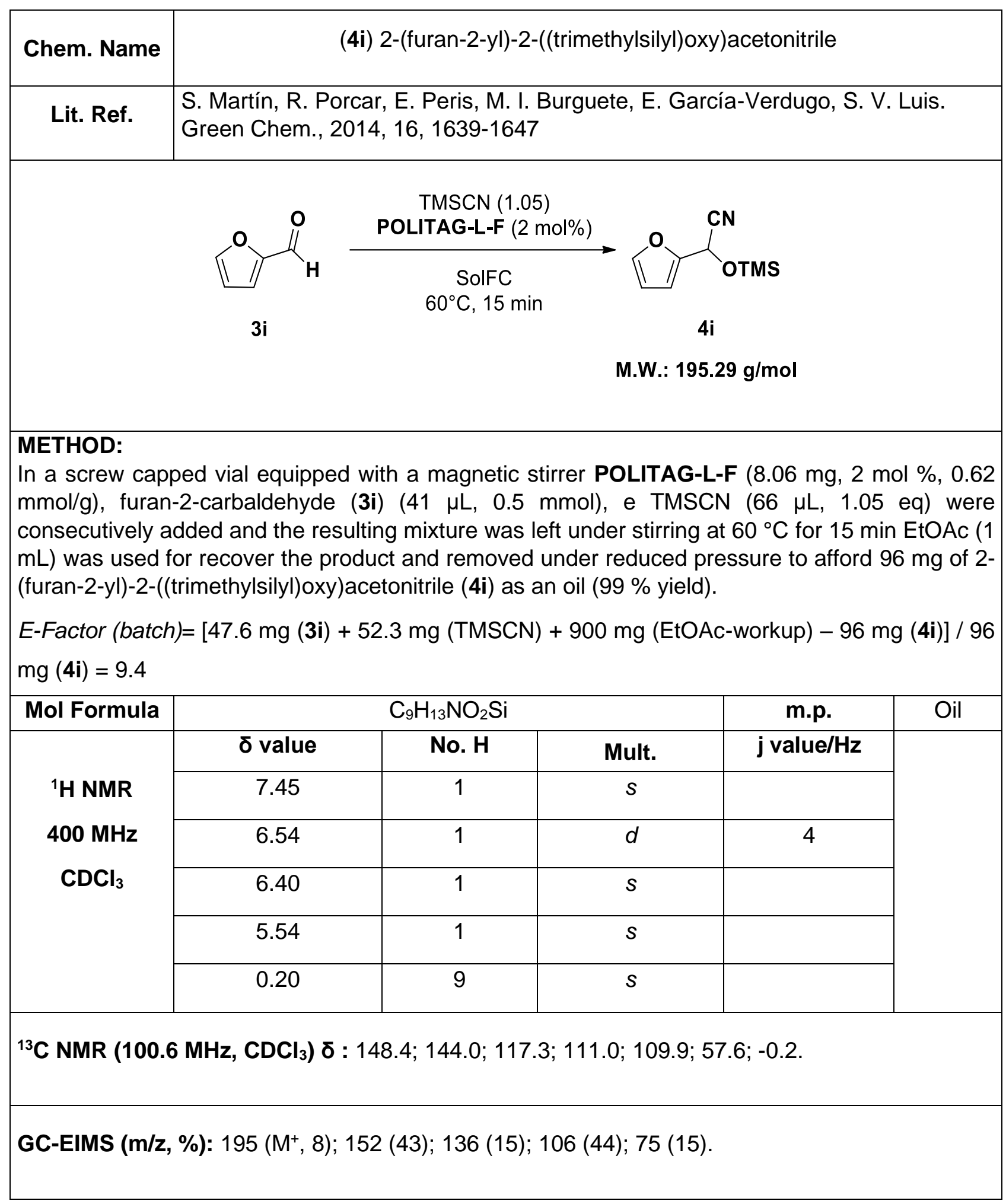




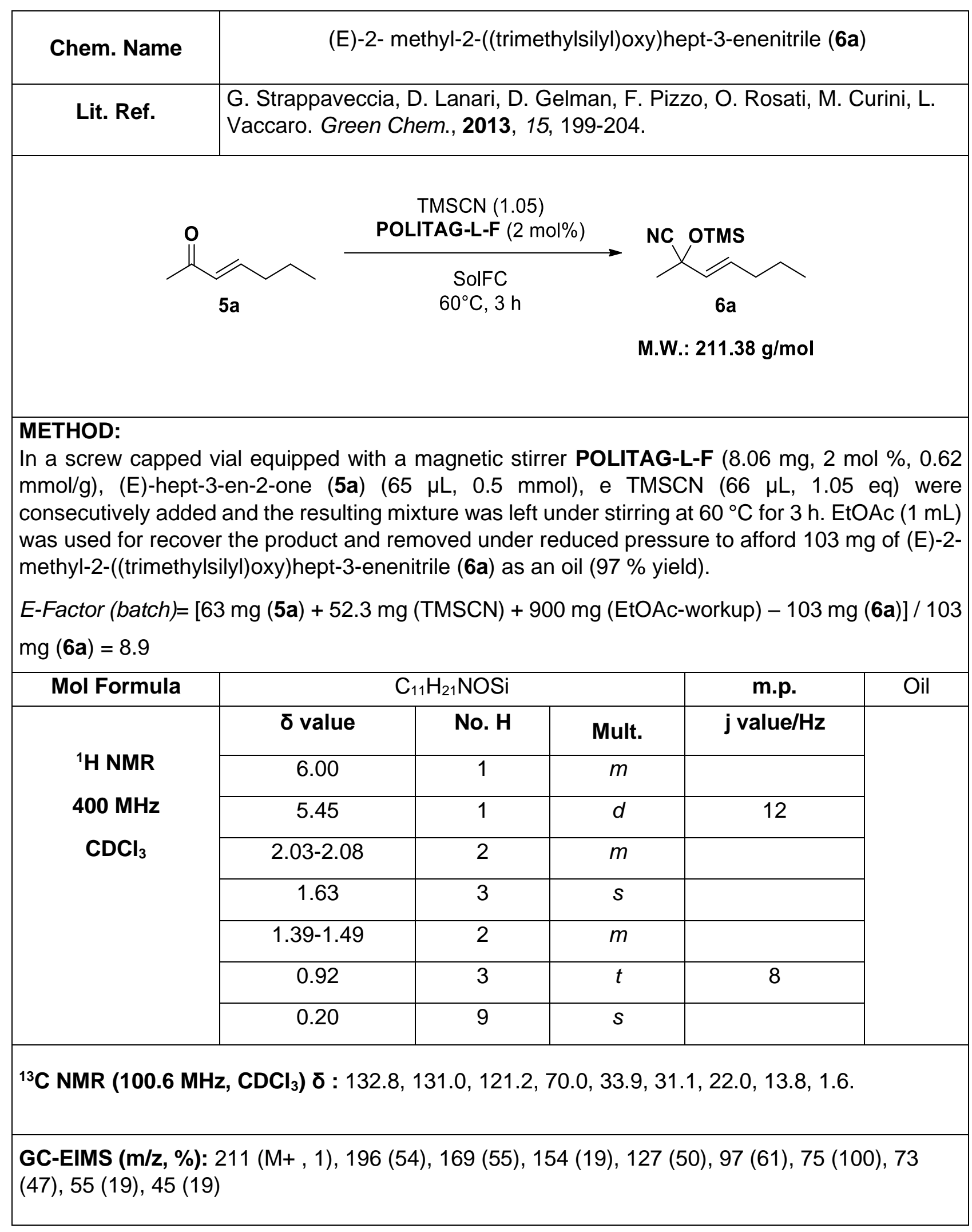




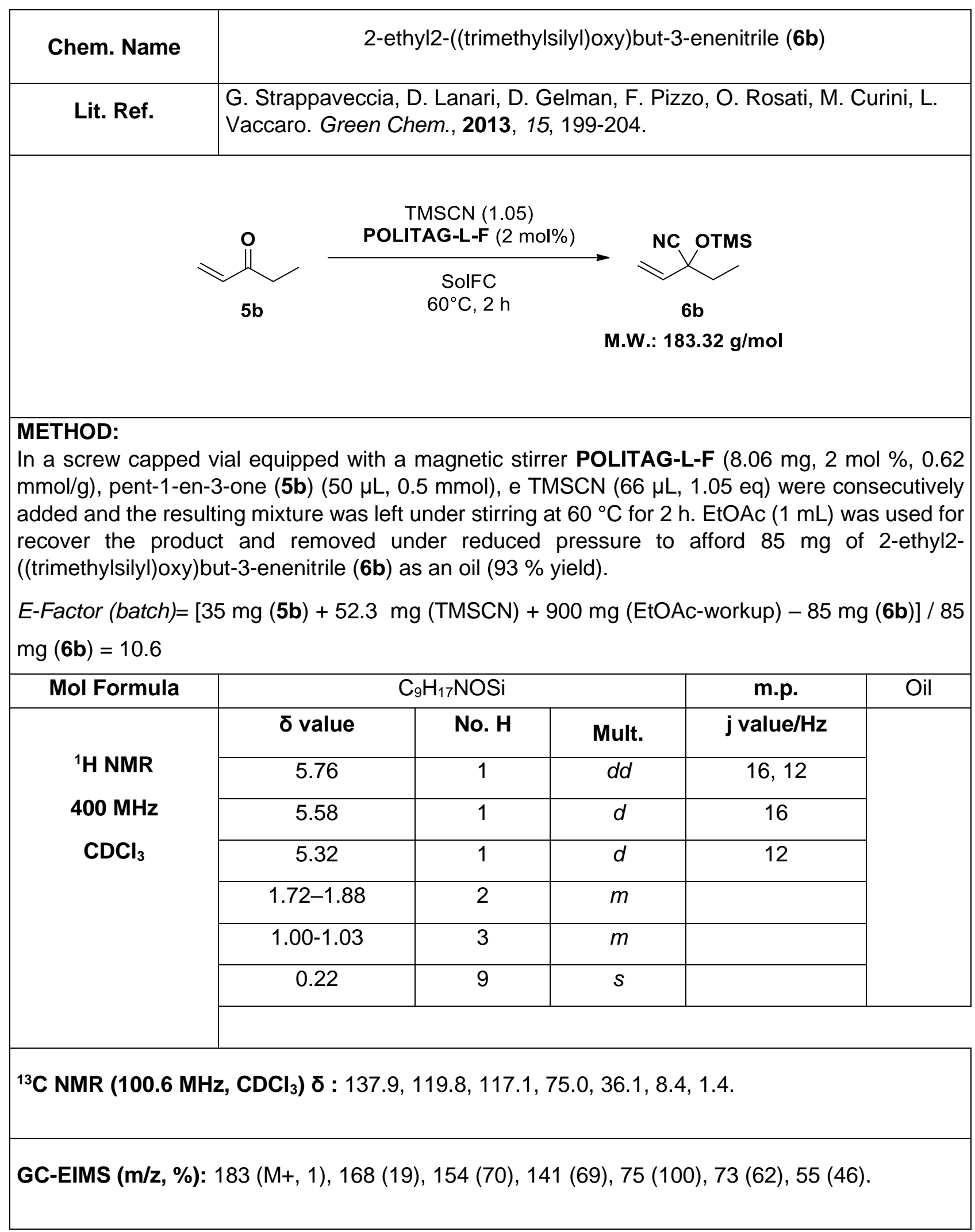




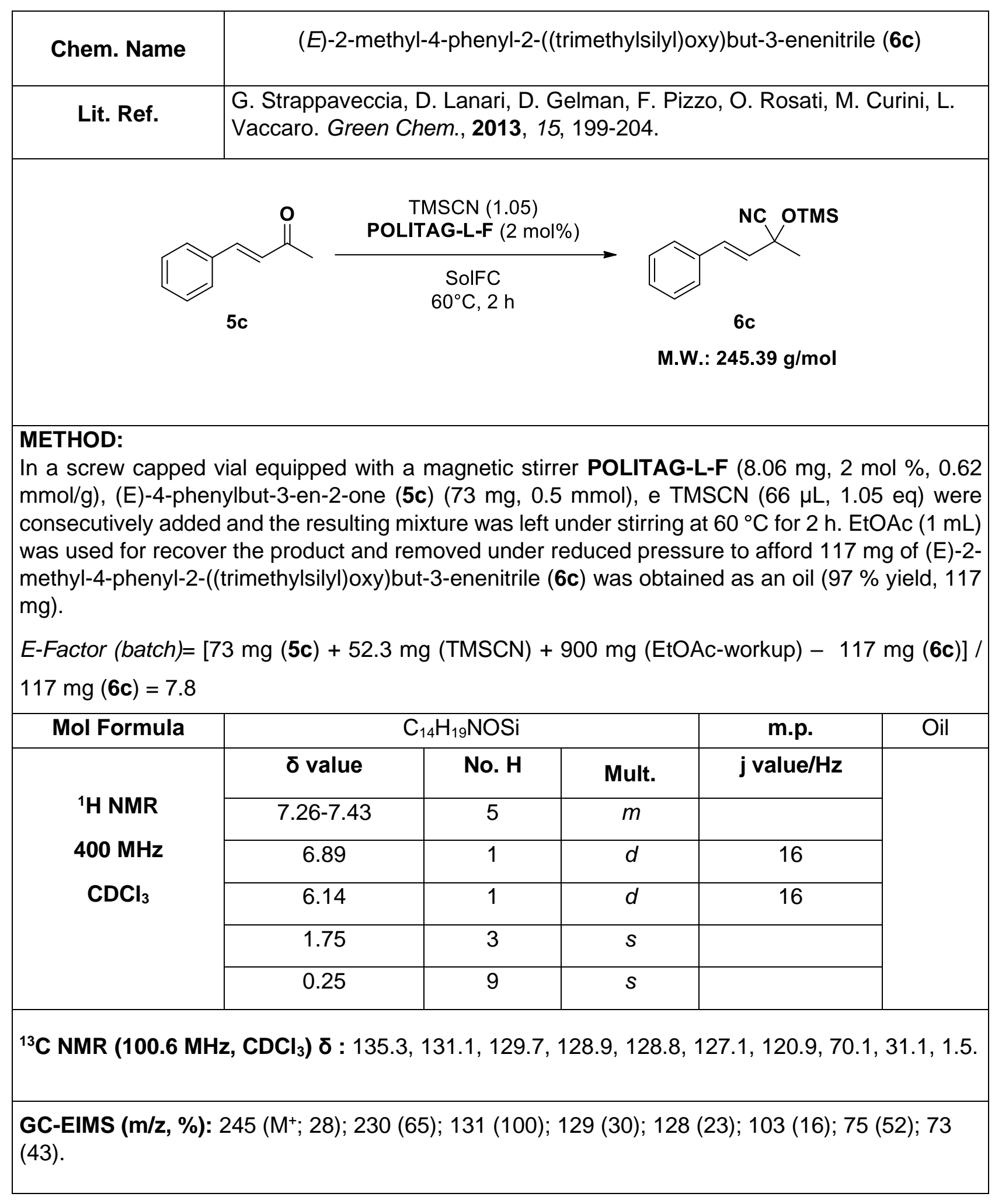




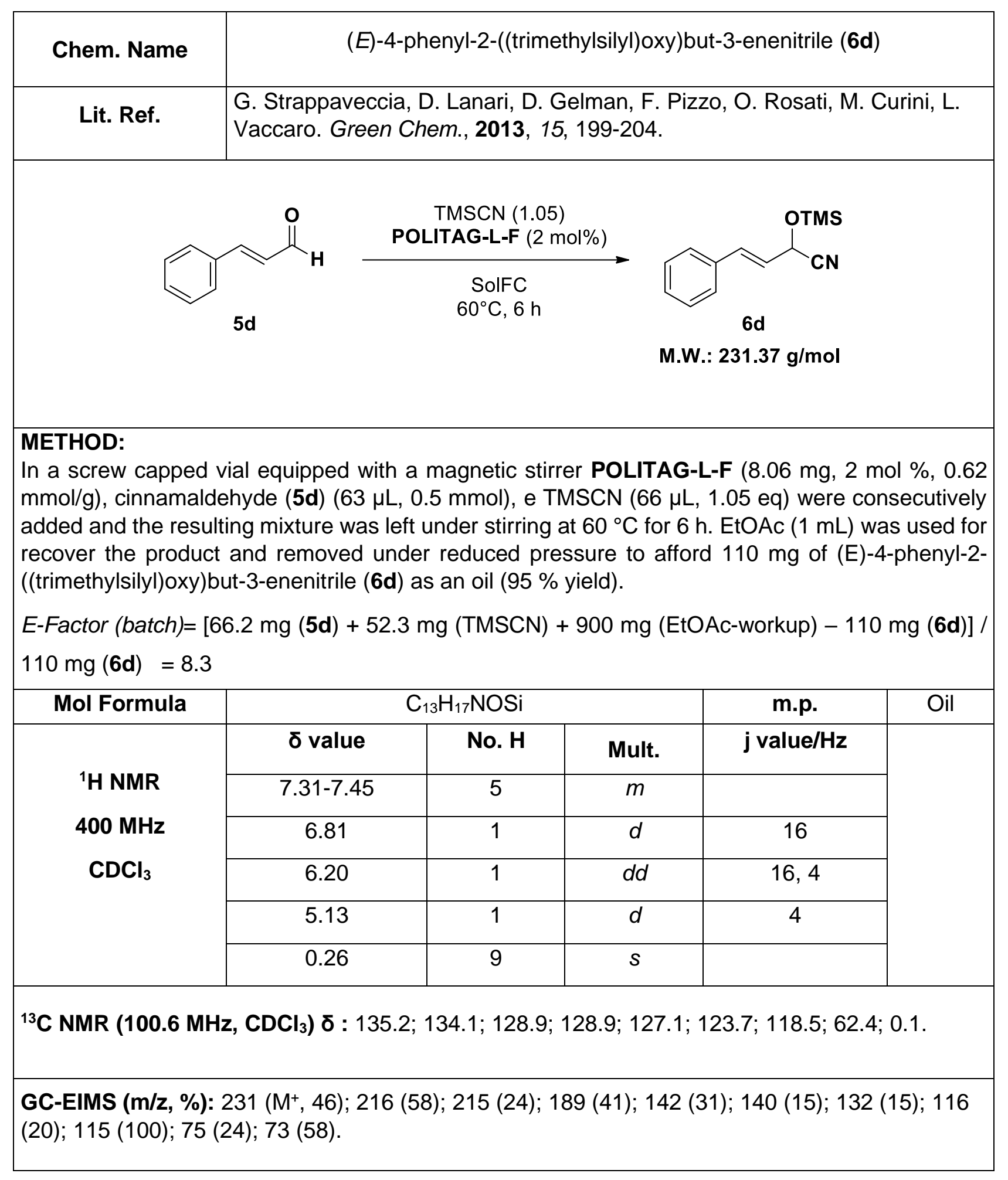


2a 2-phenyl-2-((trimethylsilyl)oxy)propanenitrile<smiles>COC(C)(C#N)c1ccccc1</smiles>

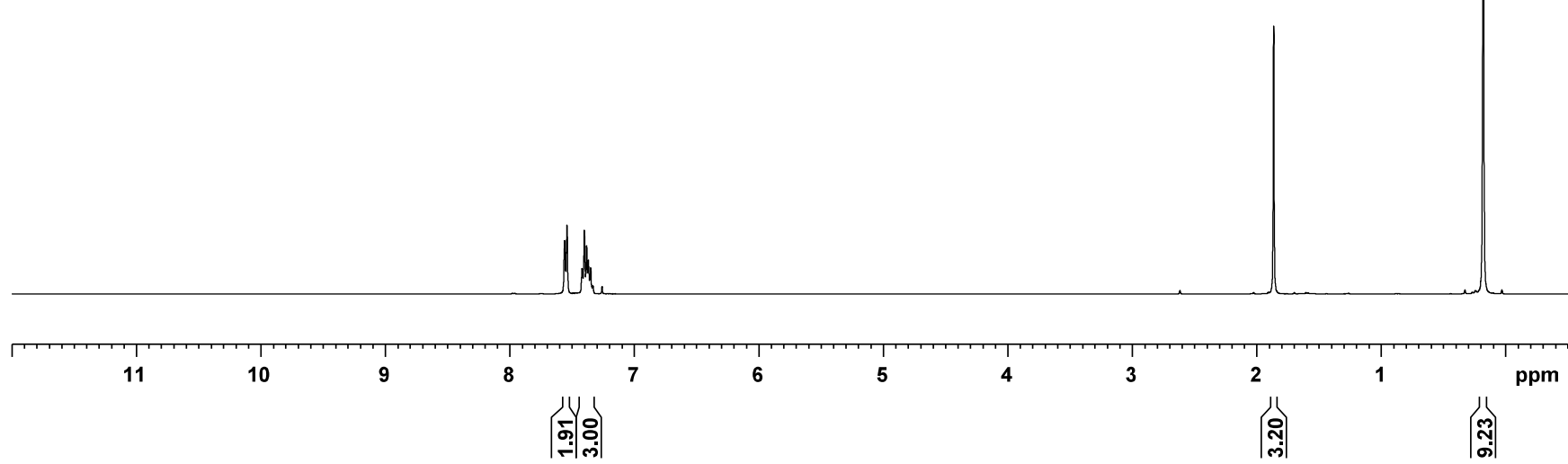

2a 2-phenyl-2-((trimethylsilyl)oxy)propanenitrile<smiles>COC(C)(C#N)c1ccccc1</smiles>

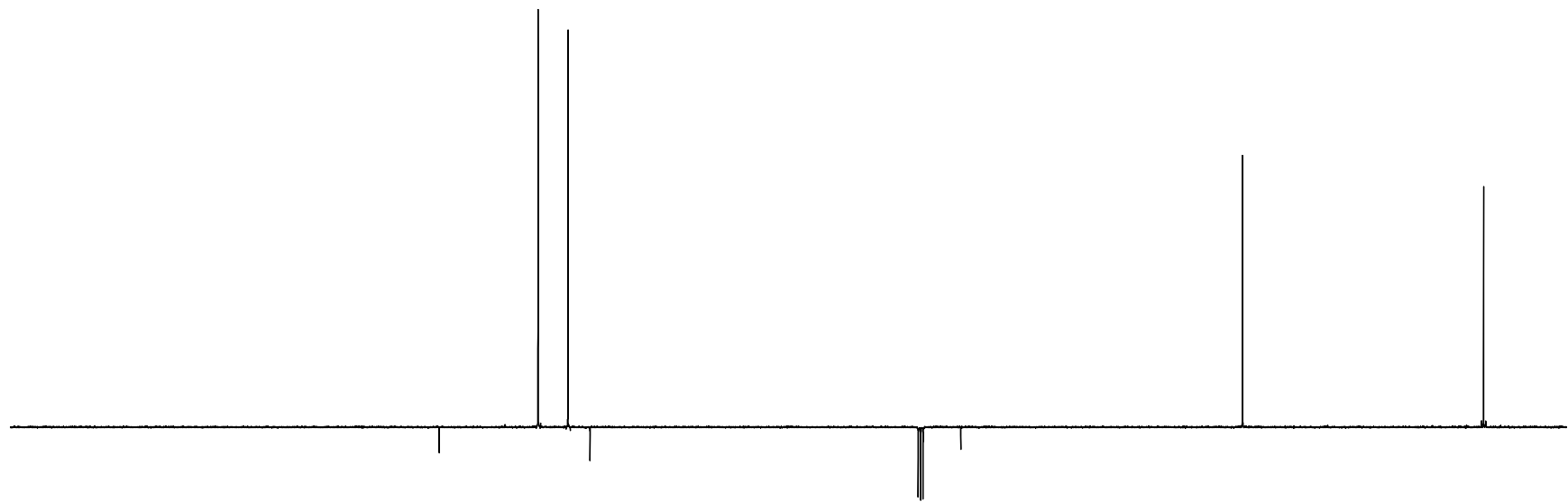


2b 2-(p-tolyl)-2-((trimethylsilyl)oxy)propanenitrile<smiles>COC(C)(C#N)c1ccc(C)cc1</smiles>

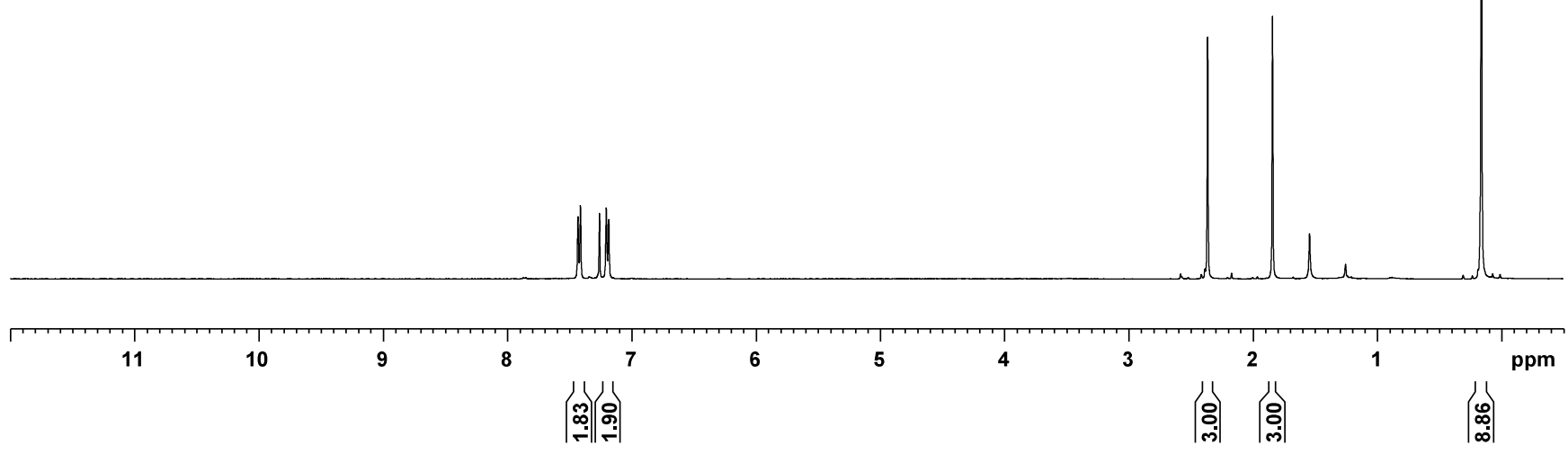

2b 2-(p-tolyl)-2-((trimethy|silyl)oxy)propanenitrile
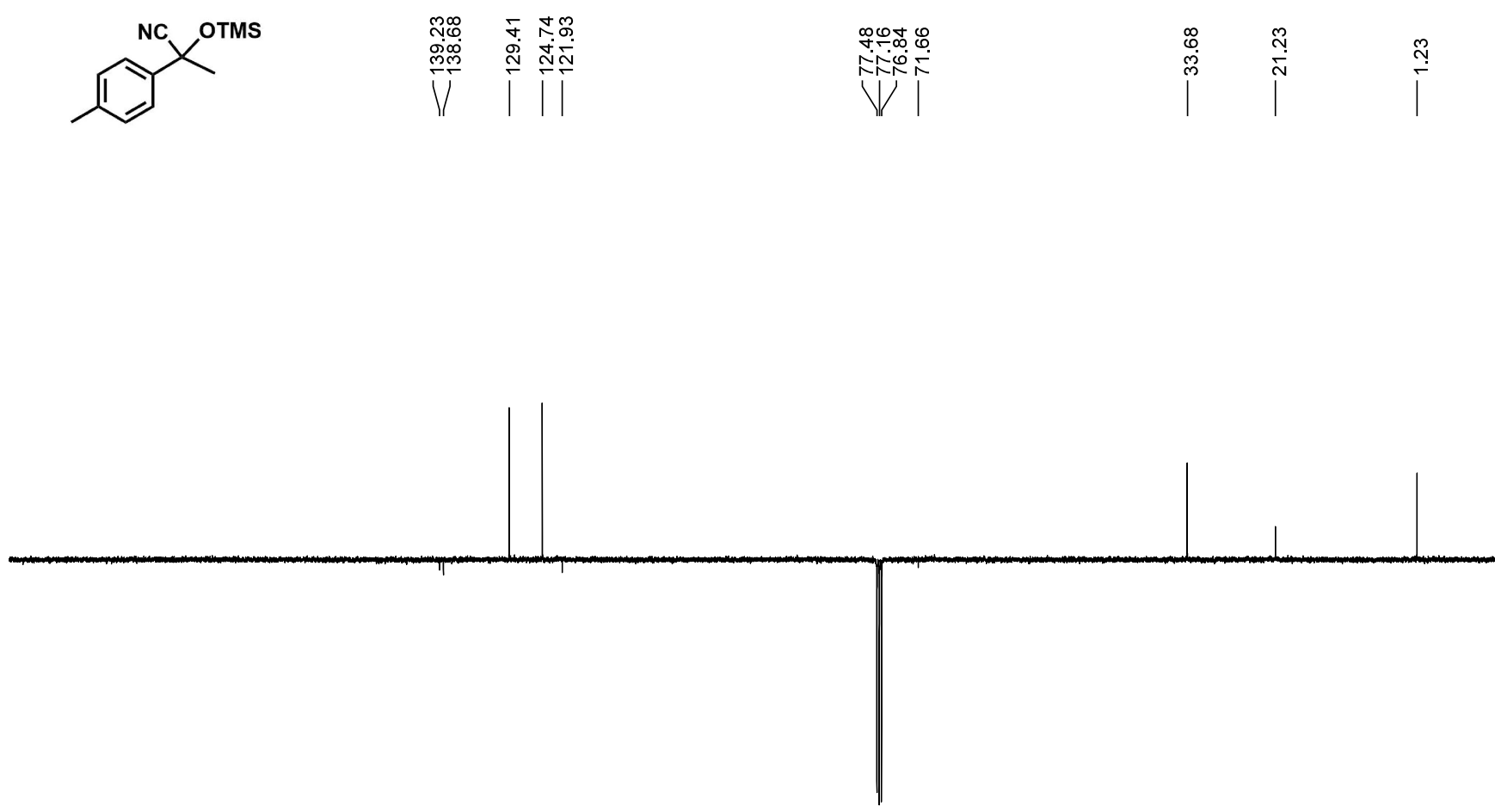
2c 2-(4-methoxyphenyl)-2-((trimethylsilyl)oxy)propanenitrile
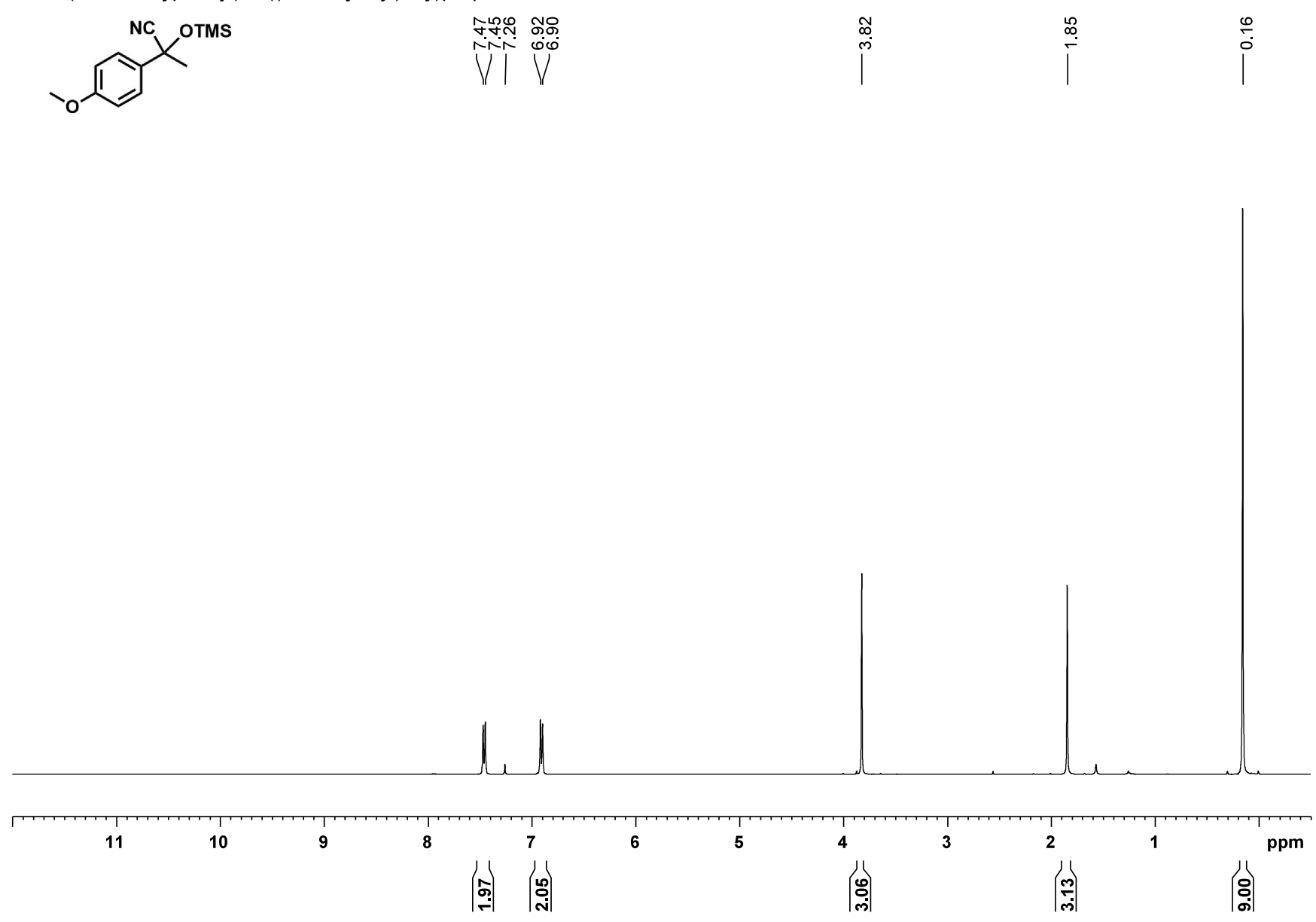

2c 2-(4-methoxyphenyl)-2-((trimethylsilyl)oxy)propanenitrile
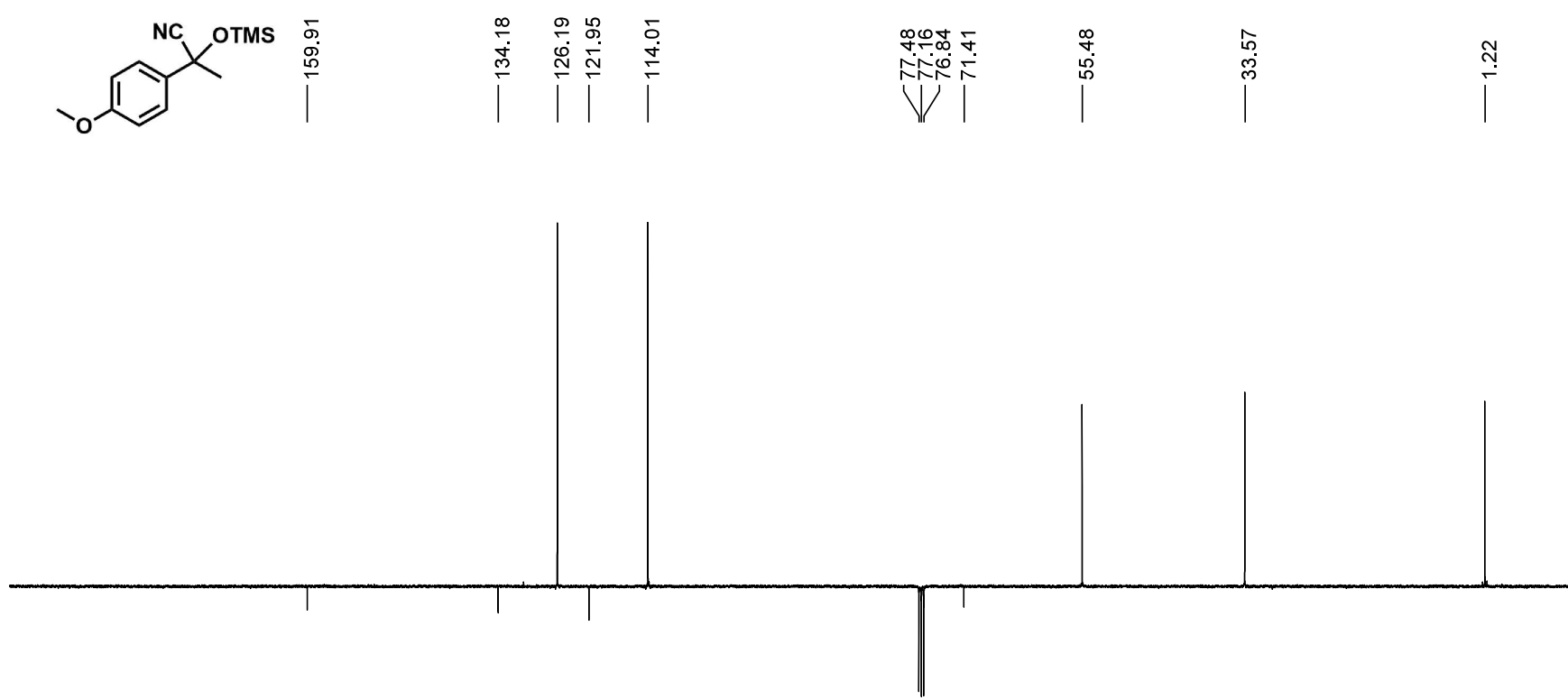
2d 2-(4-chlorophenyl)-2-((trimethylsilyl)oxy)propanenitrile<smiles>COC(C)(C)c1ccc(Cl)cc1</smiles>

अr্লne

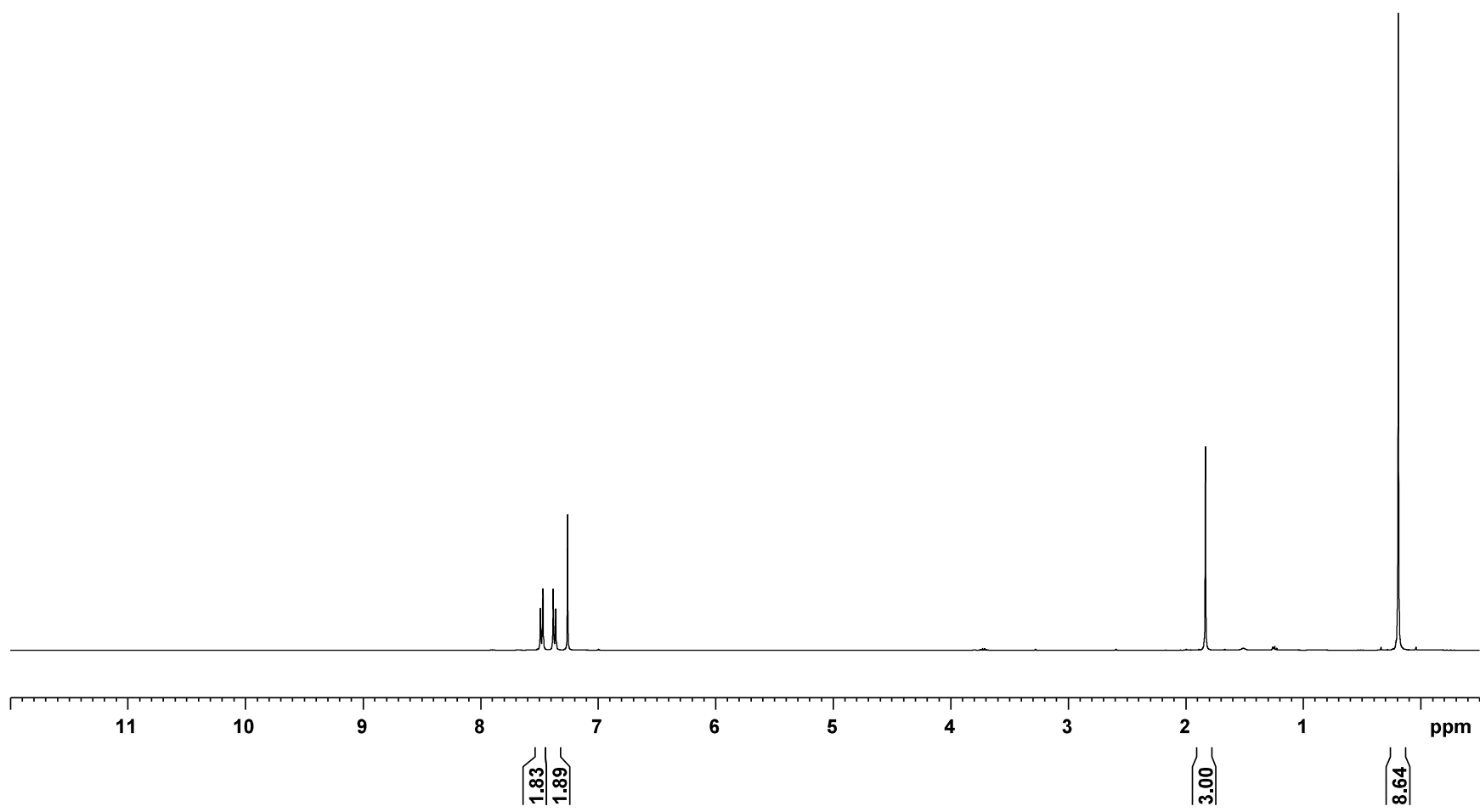

2d 2-(4-chlorophenyl)-2-((trimethylsilyl)oxy)propanenitrile

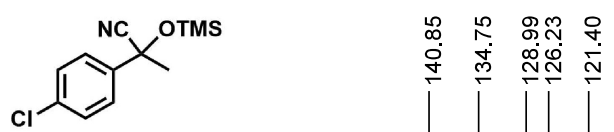

누요

수요

$\stackrel{\infty}{\stackrel{\infty}{\infty}}$

$\bar{n}$

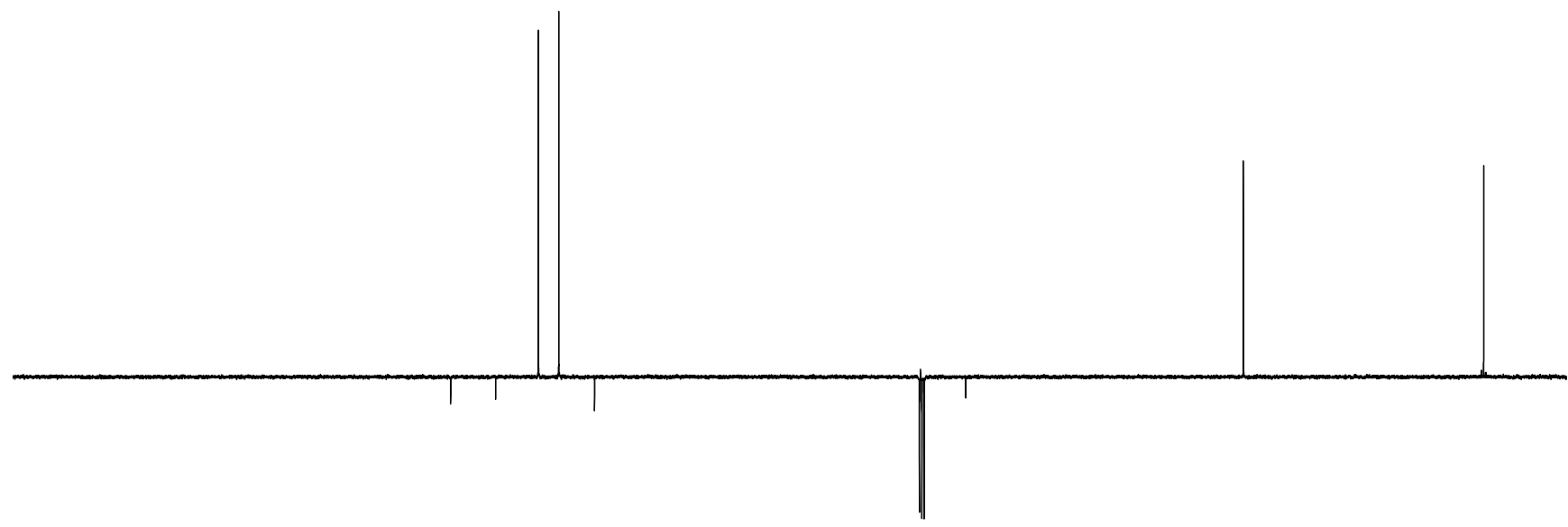



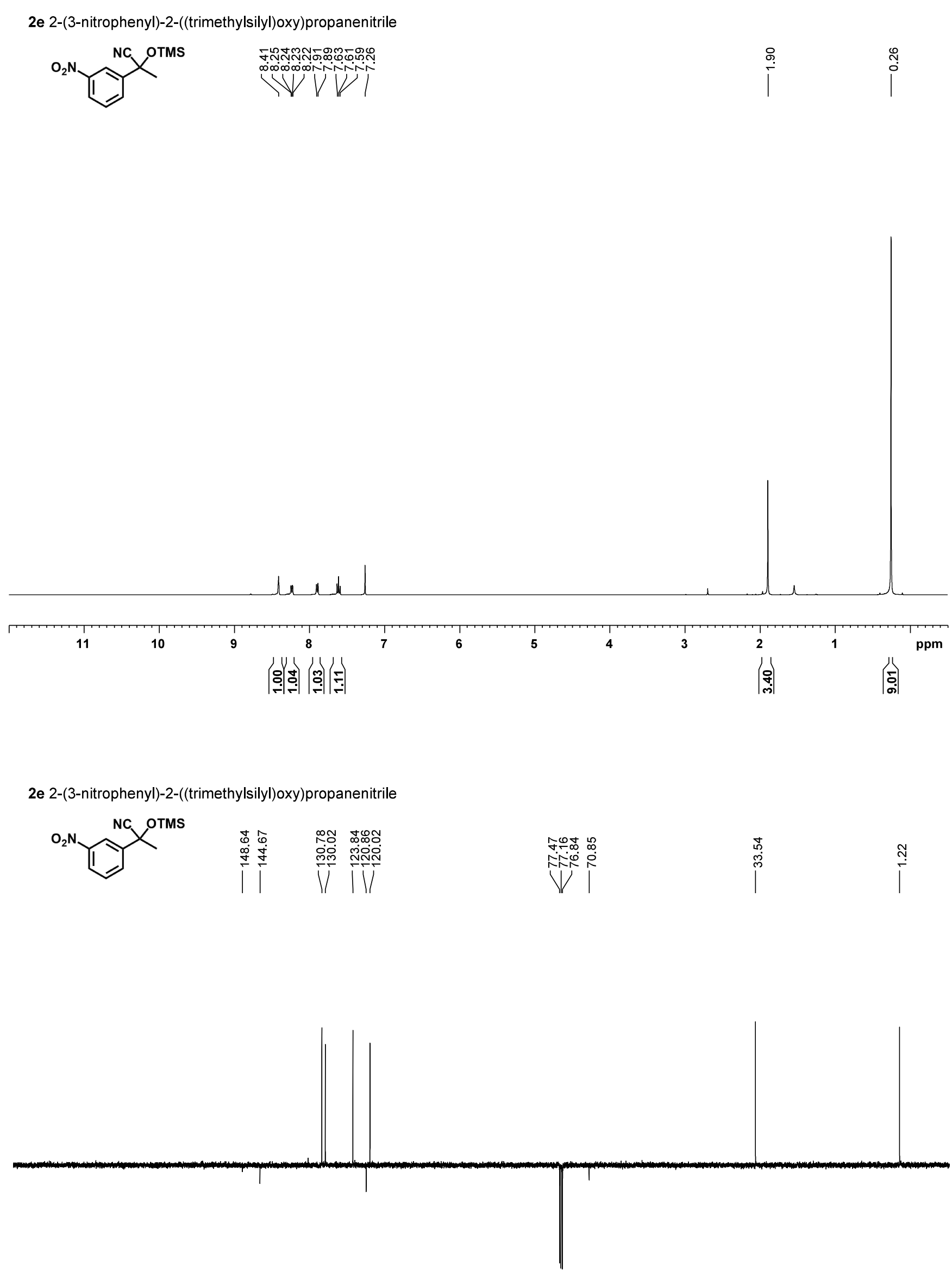
2f 1-((trimethylsilyl)oxy)-2,3-dihydro-1H-indene-1-carbonitrile

отмs

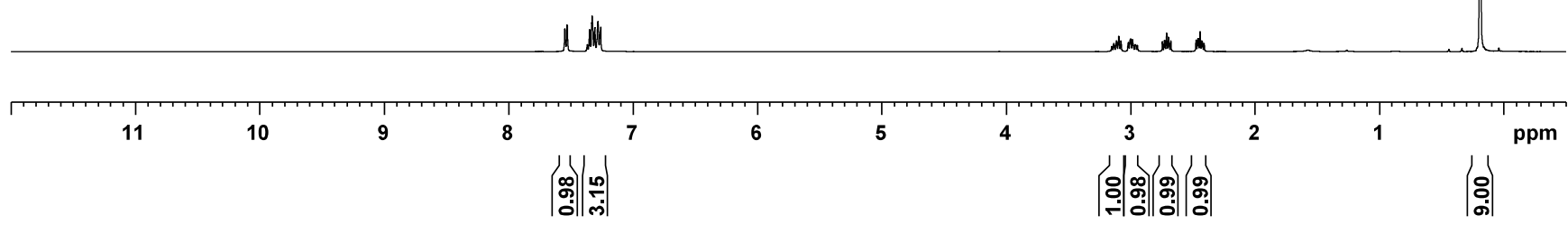

2f 1-((trimethylsilyl)oxy)-2,3-dihydro-1H-indene-1-carbonitrile

(2)

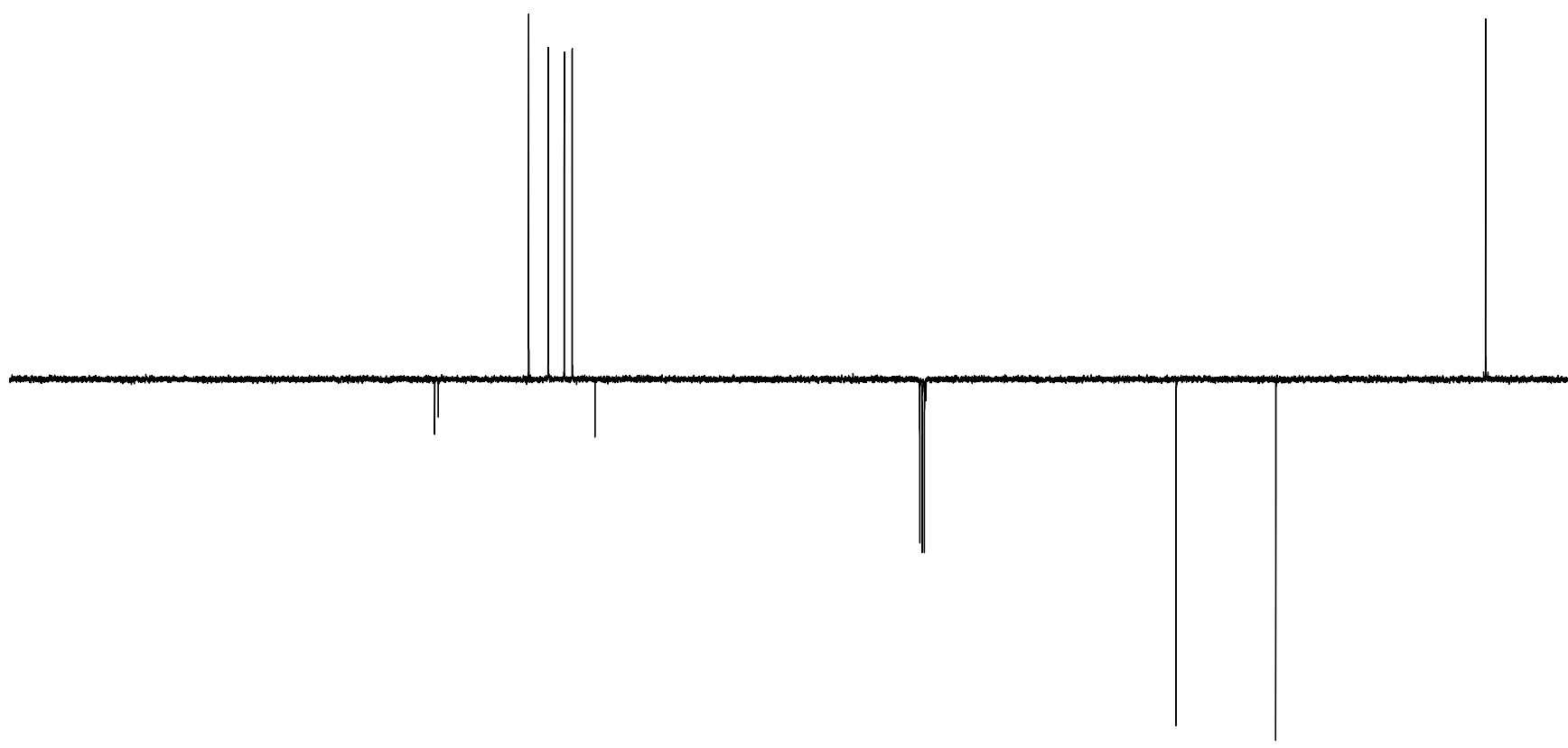


2g 2,2-diphenyl-2-((trimethylsilyl)oxy)acetonitrile
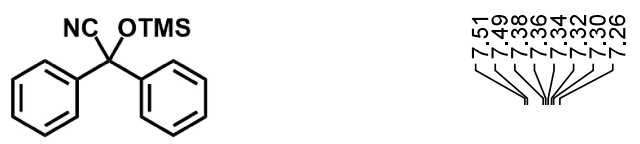

$\stackrel{+}{i}$
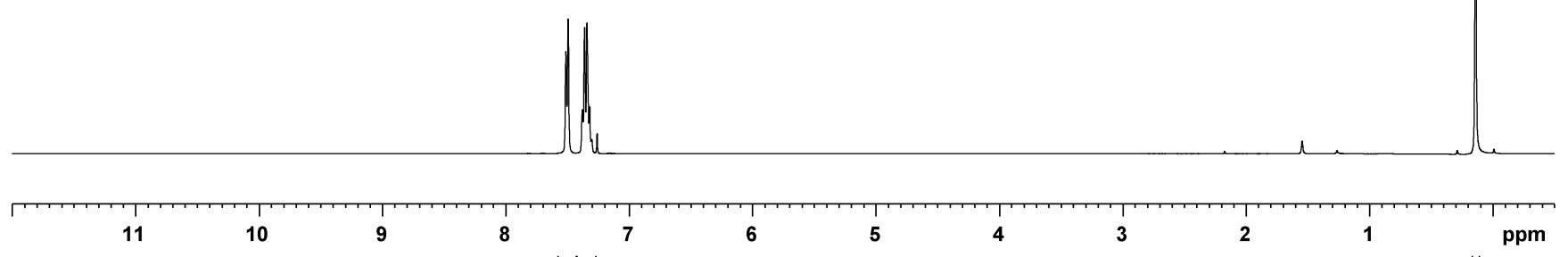

$\left|\begin{array}{l}\infty \\ 0 \\ m\end{array}\right| \begin{gathered}\mathbf{m} \\ 0\end{gathered} \mid$

(⿱夂口)

2g 2,2-diphenyl-2-((trimethylsilyl)oxy)acetonitrile
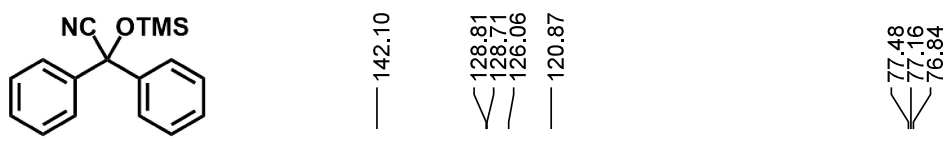

$\stackrel{\text { ó }}{i}$

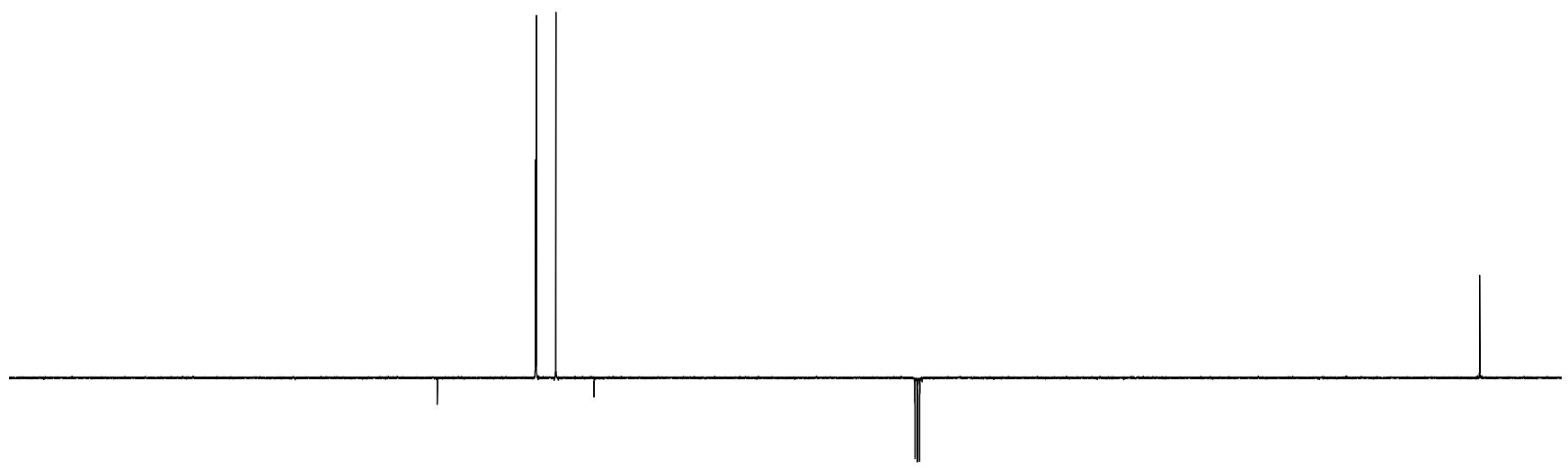

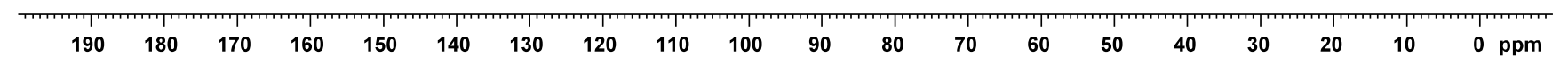

S39 
2h 2-(pyridin-2-yl)-2-((trimethylsilyl)oxy)propanenitrile
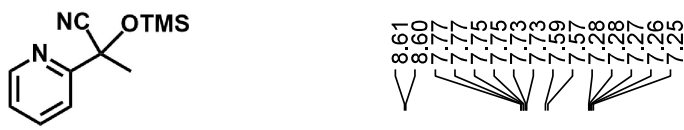

$\stackrel{5}{i}$

$\stackrel{\text { i }}{\circ}$

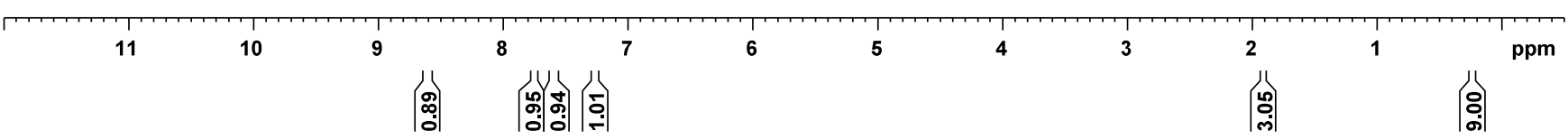

2h 2-(pyridin-2-yl)-2-((trimethylsilyl)oxy)propanenitrile

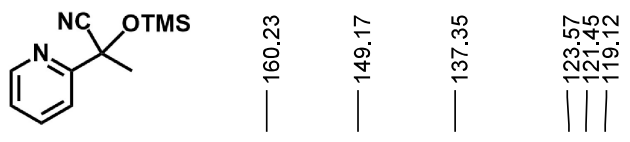

inis
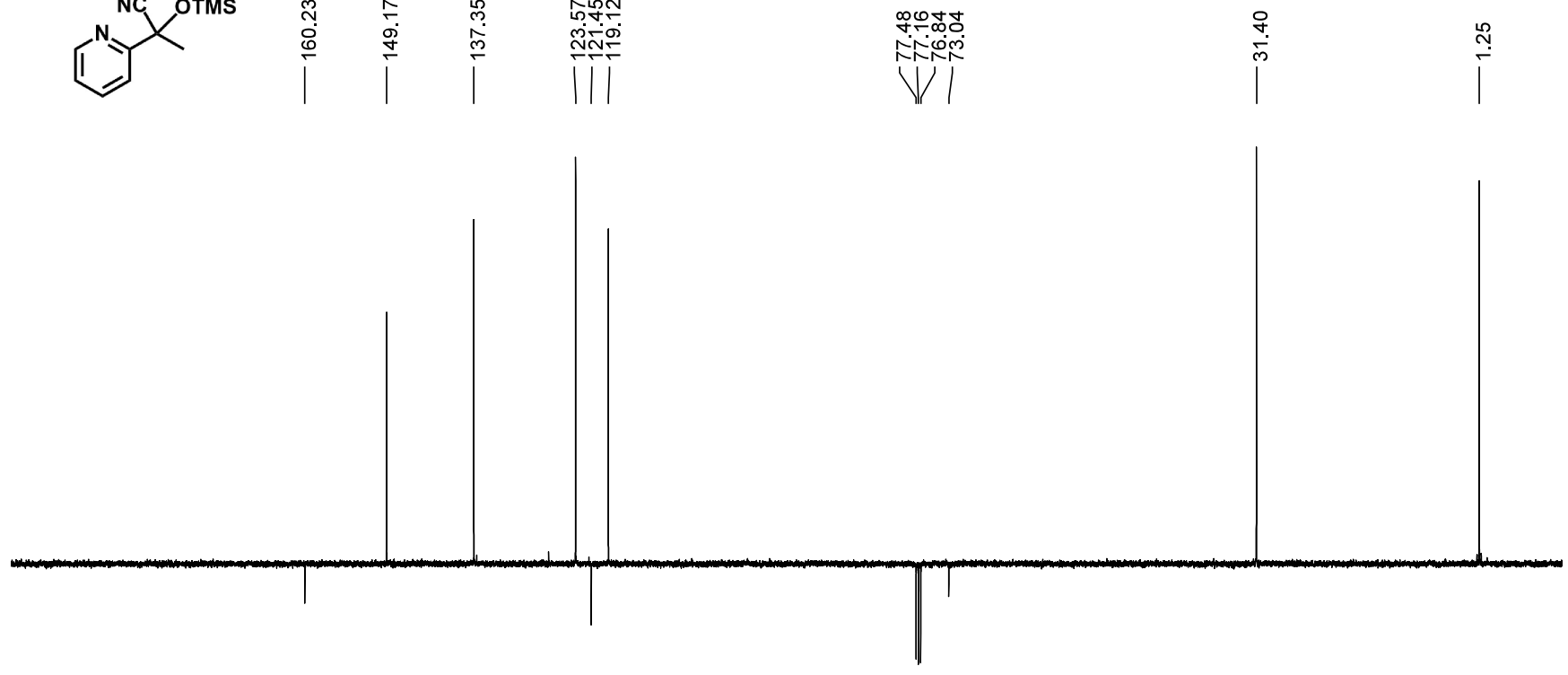

190

$\begin{array}{lllllllllllll}180 & 170 & 160 & 150 & 140 & 130 & 120 & 110 & 100 & 90 & 80 & 70 & 60\end{array}$

$50 \quad 40$

30

20

0 ppm

S40 
2i 2-methyl-2-((trimethylsilyl)oxy)octanenitrile
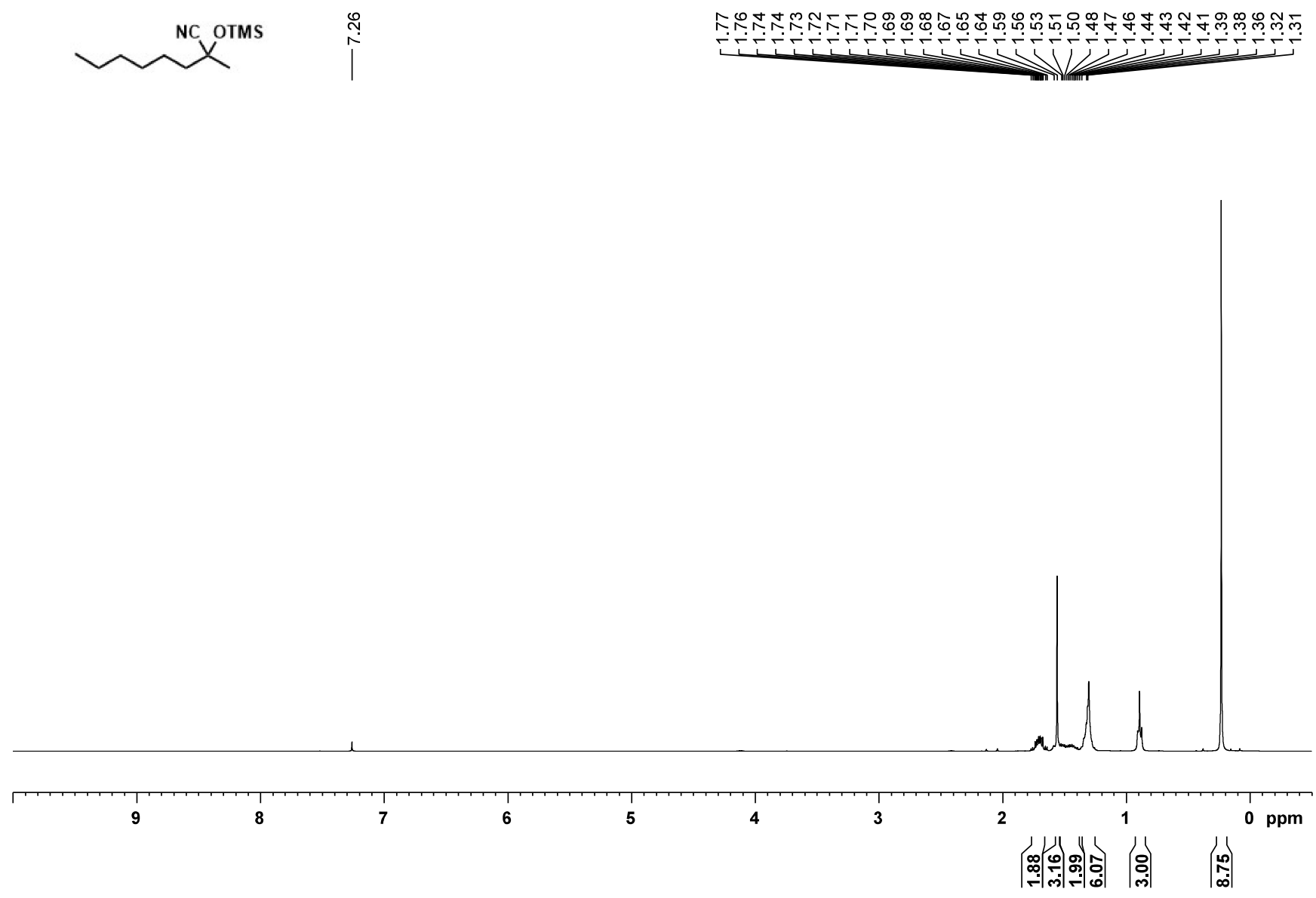

2i 2-methyl-2-((trimethylsilyl)oxy)octanenitrile

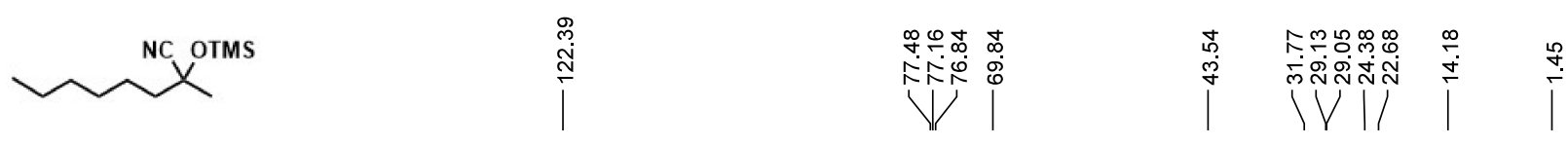

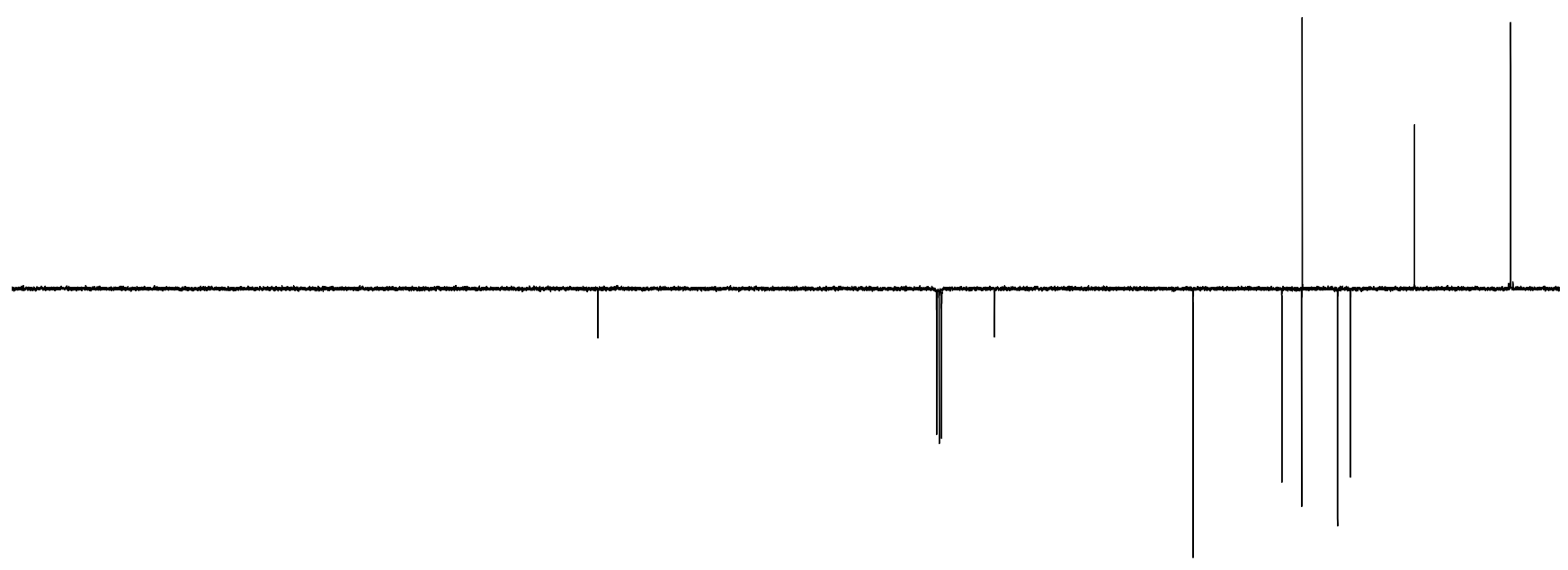


2j 1-((trimethylsilyl)oxy)cyclohexanecarbonitrile<smiles>COC1(C#N)CCCCC1</smiles>

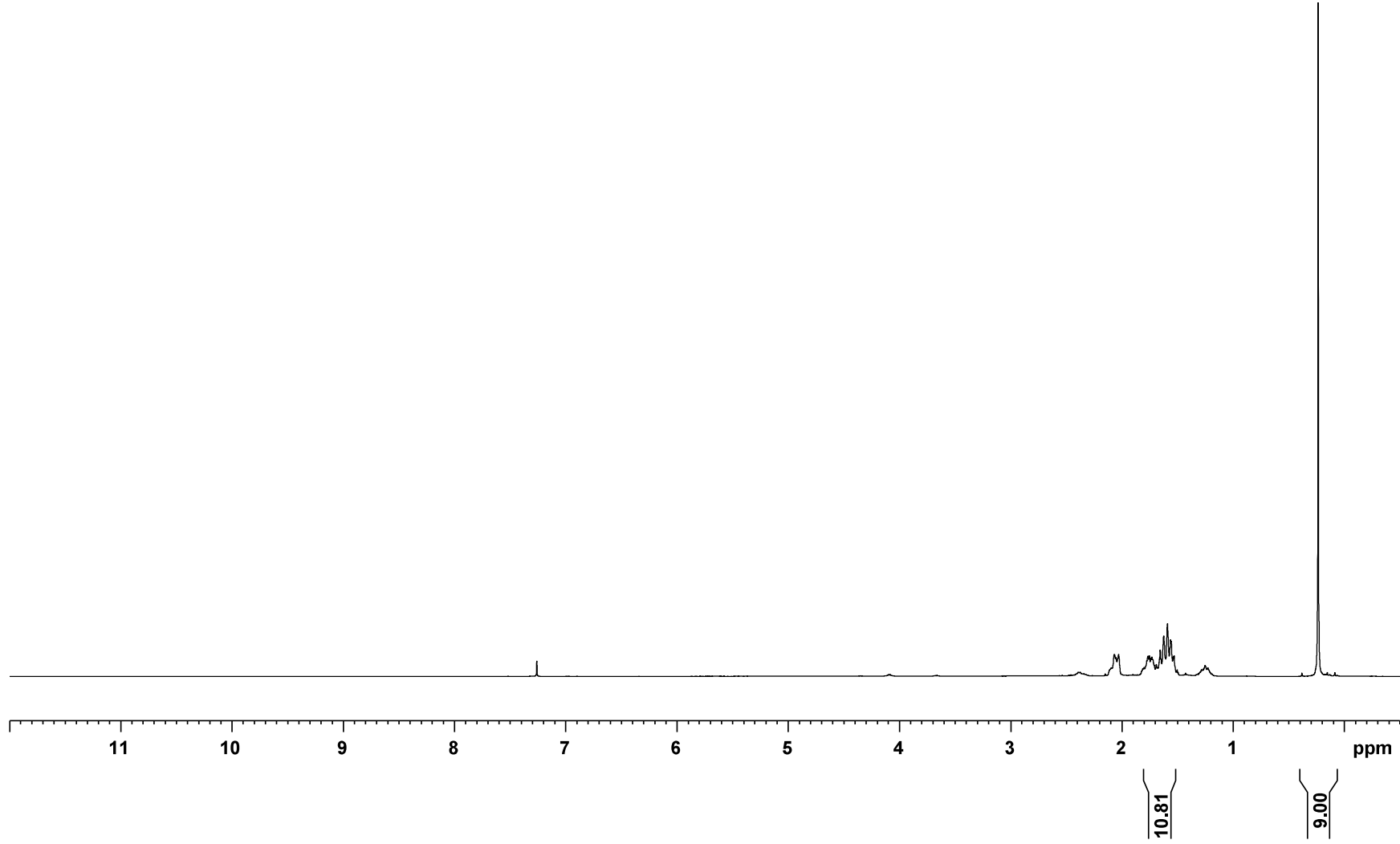

2j 1-((trimethylsilyl)oxy)cyclohexanecarbonitrile<smiles>COC1(C#N)CCCCC1</smiles> 
4a 2-phenyl-2-((trimethylsilyl)oxy)acetonitrile<smiles>C#CC(O[Na])c1ccccc1</smiles>

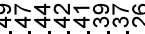

ininitini

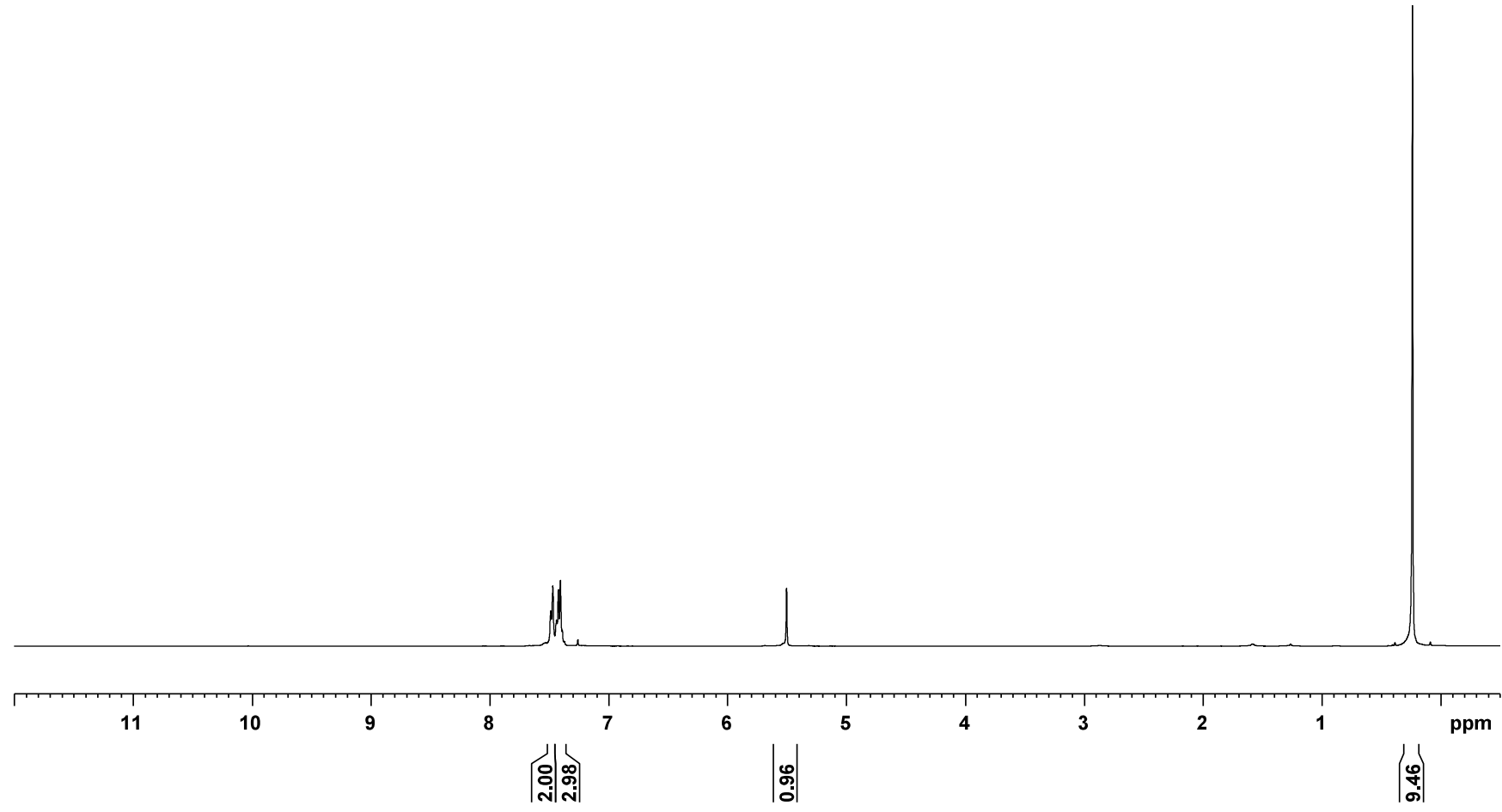

4a 2-phenyl-2-((trimethylsilyl)oxy)acetonitrile<smiles>COC(C#N)c1ccccc1</smiles>

i ivi

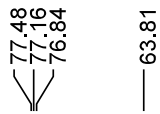

ז'

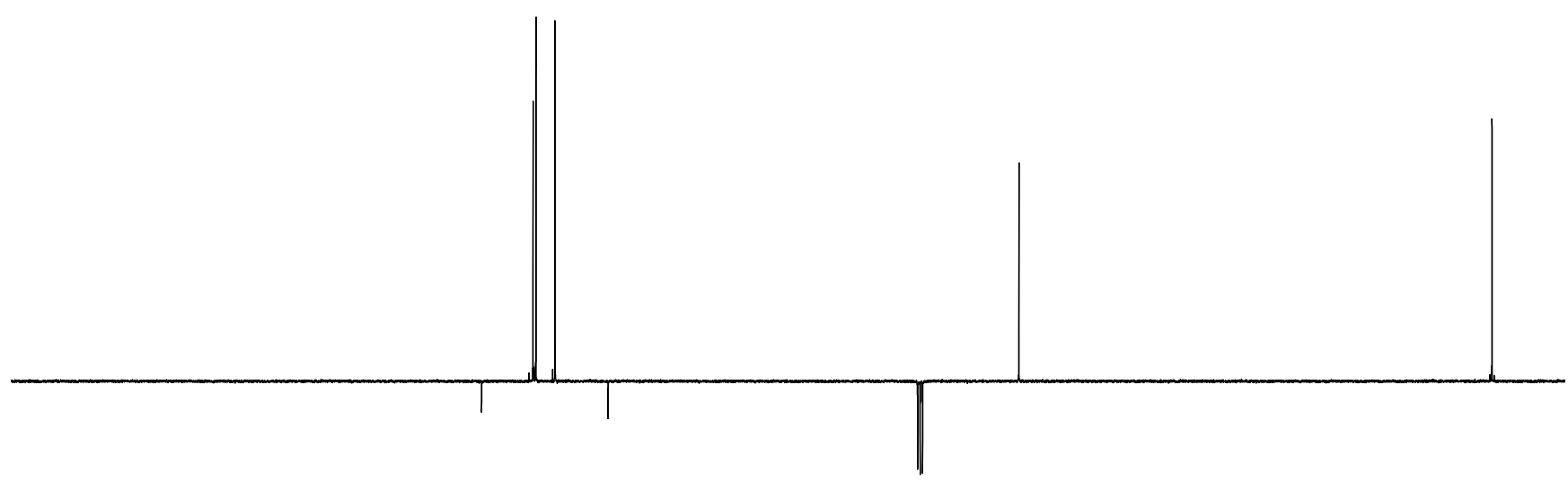

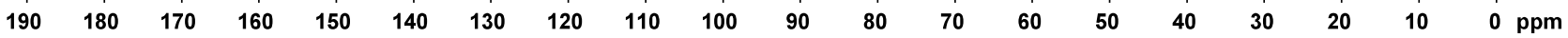


4b 2-(4-nitrophenyl)-2-((trimethylsilyl)oxy)acetonitrile

OTMs

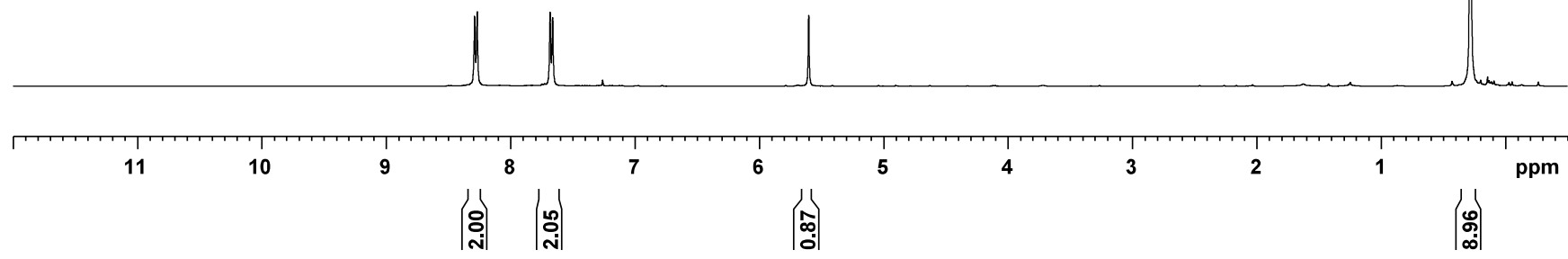

4b 2-(4-nitrophenyl)-2-((trimethylsilyl)oxy)acetonitrile
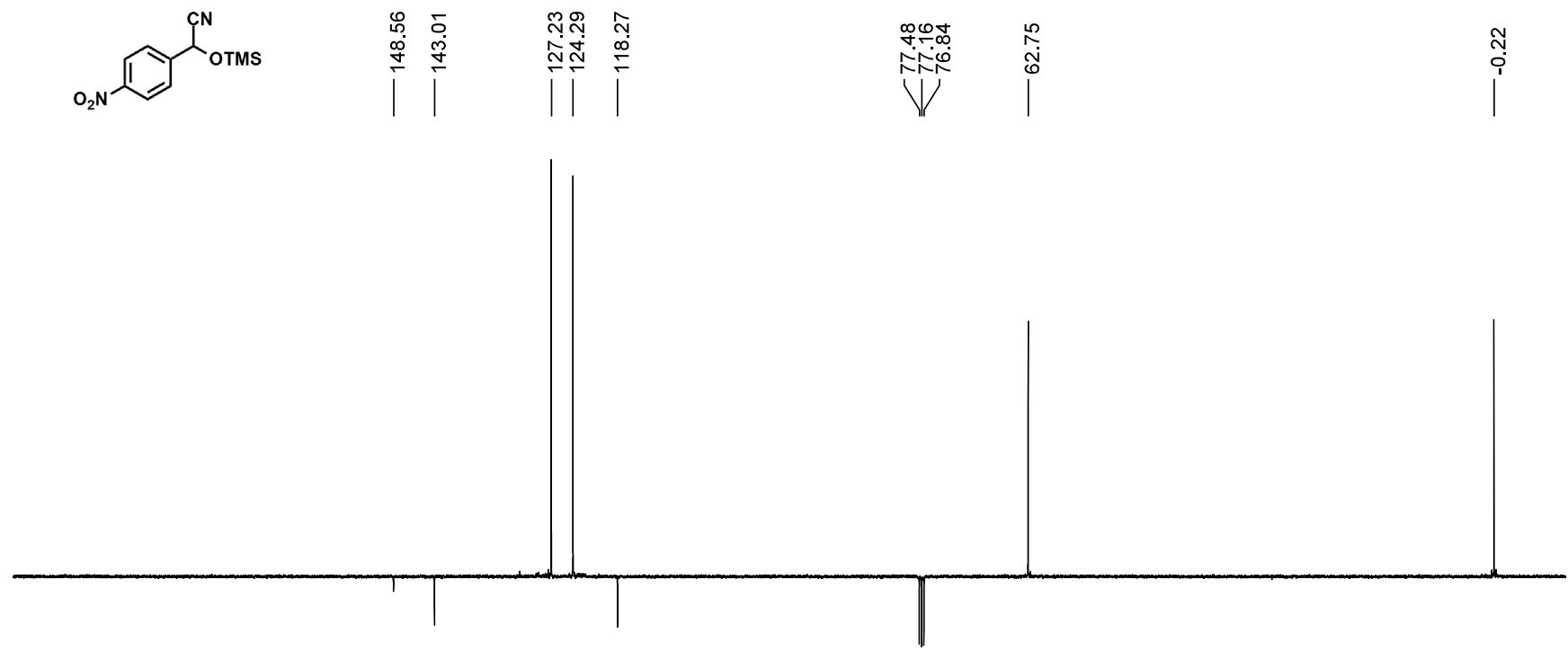

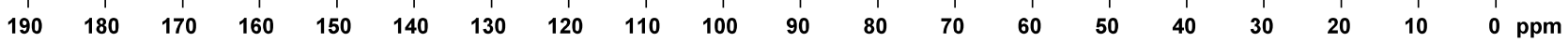


4c 3-(cyano((trimethylsilyl)oxy)methyl)benzonitrile<smiles>COC(C#N)c1cccc(C#N)c1</smiles>

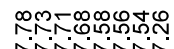

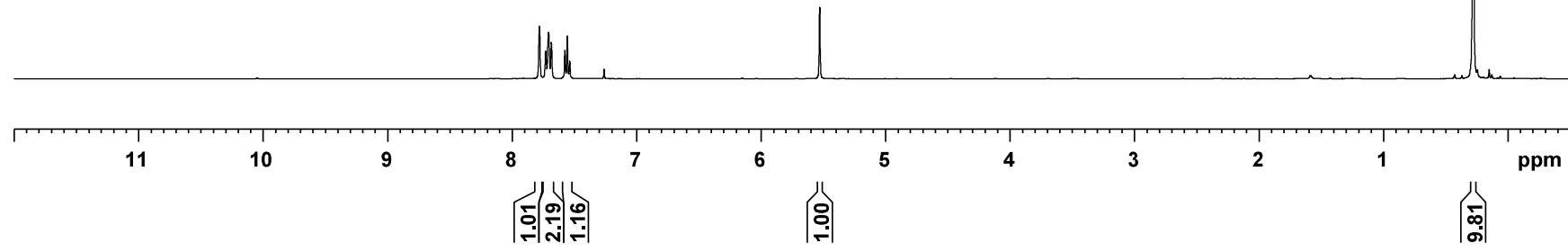

4c 3-(cyano((trimethy|silyl)oxy)methyl)benzonitrile<smiles>COC(C#N)c1cccc(C#N)c1</smiles>

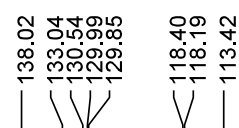

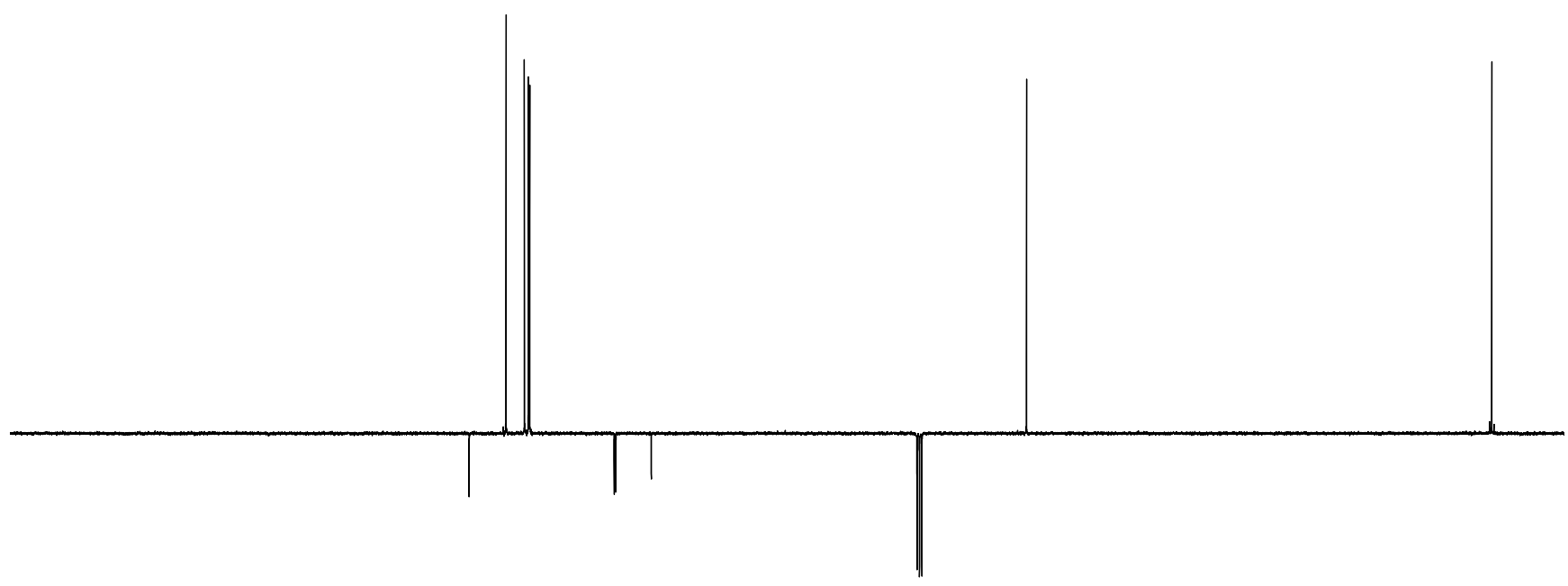


4d 2-(4-chlorophenyl)-2-((trimethylsilyl)oxy)acetonitrile<smiles>COC(C#N)c1ccc(Cl)cc1</smiles>

mogomen

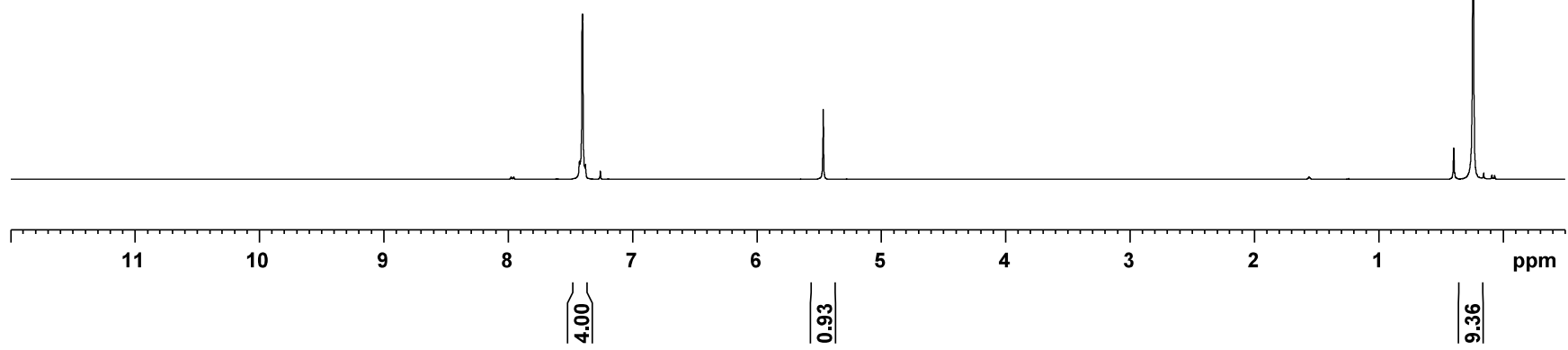

4d 2-(4-chlorophenyl)-2-((trimethylsilyl)oxy)acetonitrile<smiles>COC(C#N)c1ccc(Cl)cc1</smiles>

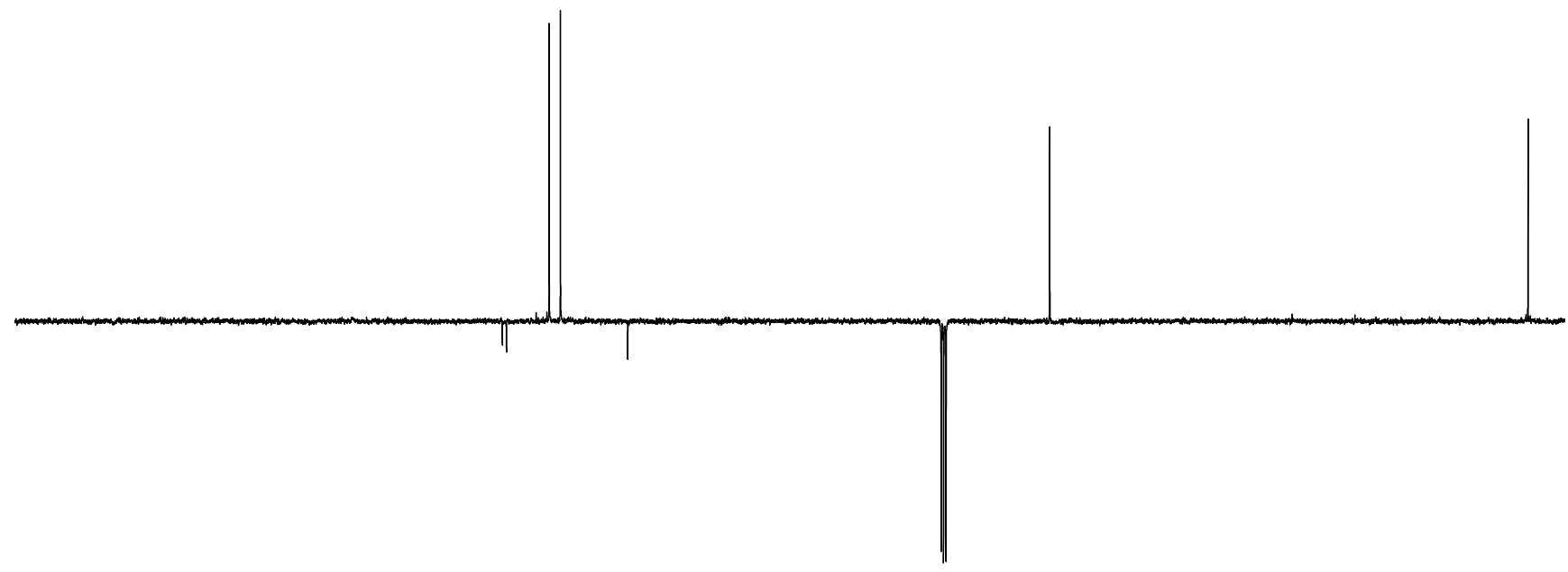


4e 2-(3-chlorophenyl)-2-((trimethylsilyl)oxy)acetonitrile<smiles>COC(C#N)c1cccc(Cl)c1</smiles>

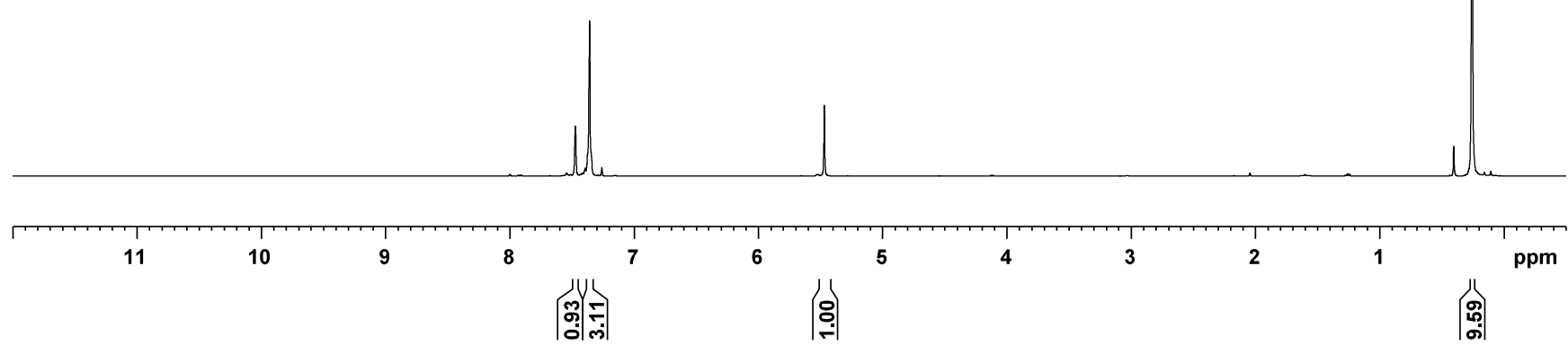

4e 2-(3-chlorophenyl)-2-((trimethylsilyl)oxy)acetonitrile<smiles>COC(C#N)c1cccc(Cl)c1</smiles>

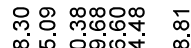

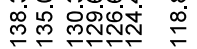

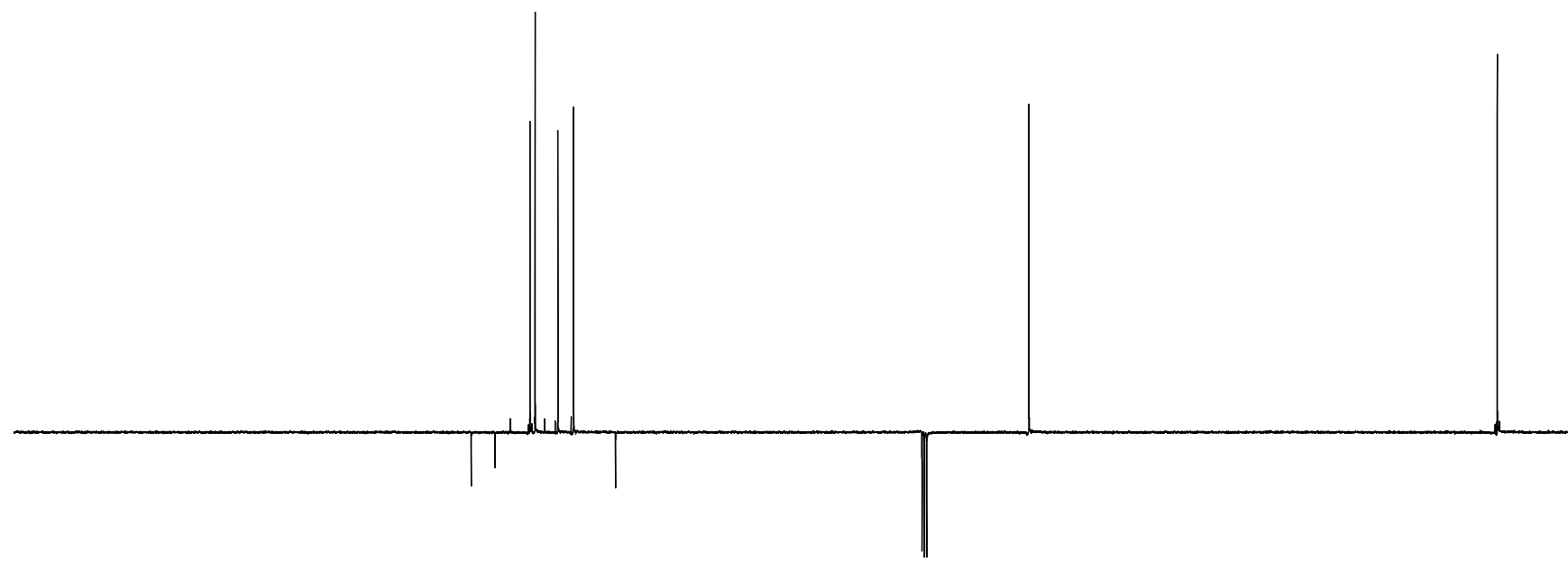


4f 2-(4-fluorophenyl)-2-((trimethylsilyl)oxy)acetonitrile

otms

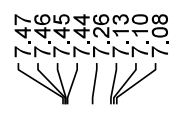

$\overbrace{\substack{f \\ 0}}^{\substack{0 \\ 0}}$

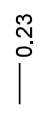

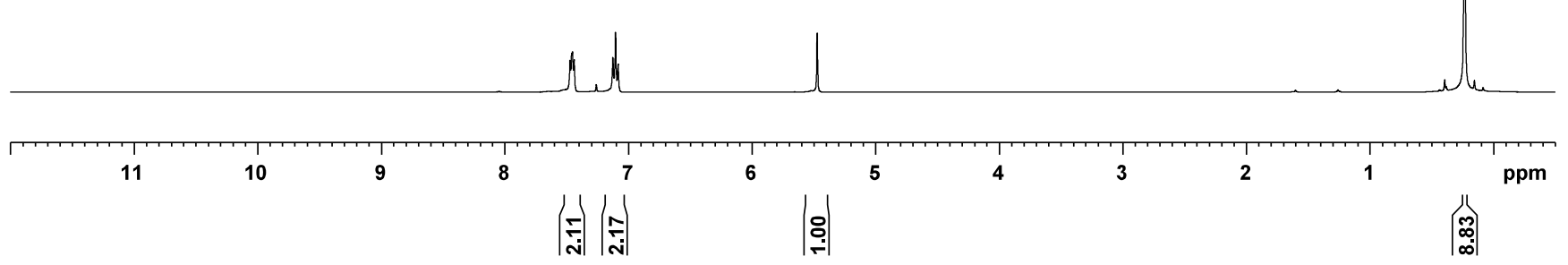

4f 2-(4-fluorophenyl)-2-((trimethylsilyl)oxy)acetonitrile
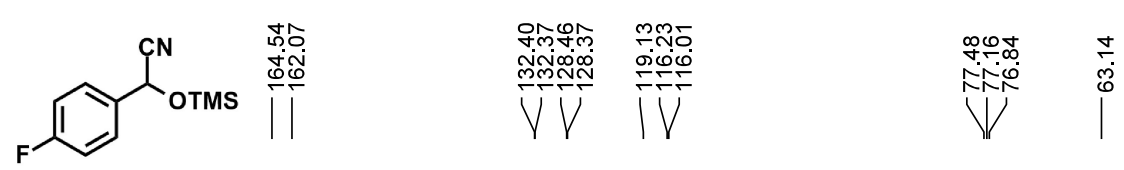

$\stackrel{m}{i}$

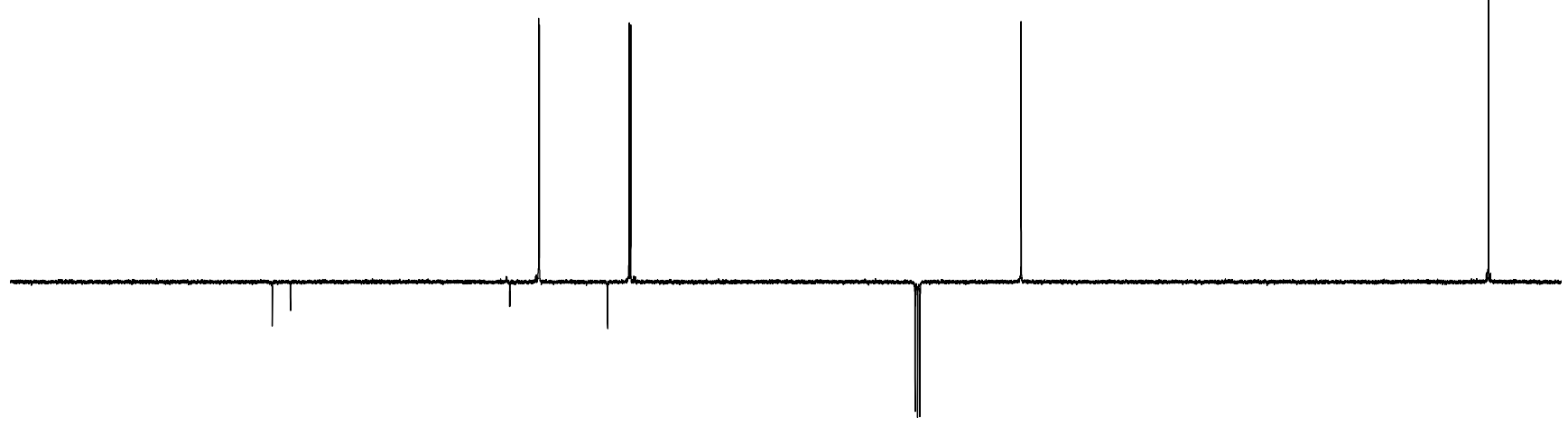

S48 
<smiles>COC(C#N)c1ccc(F)cc1</smiles>

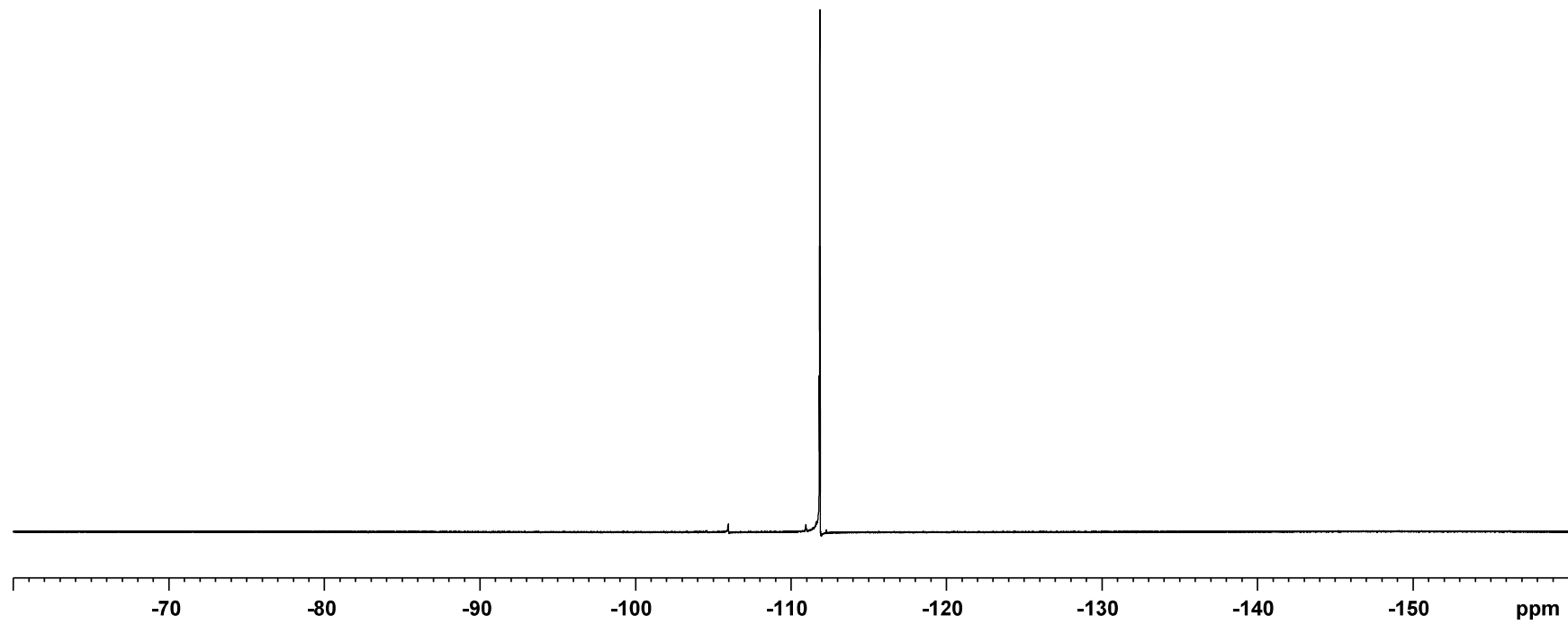

4g 2-(4-methoxyphenyl)-2-((trimethy|silyl)oxy)acetonitrile

舟

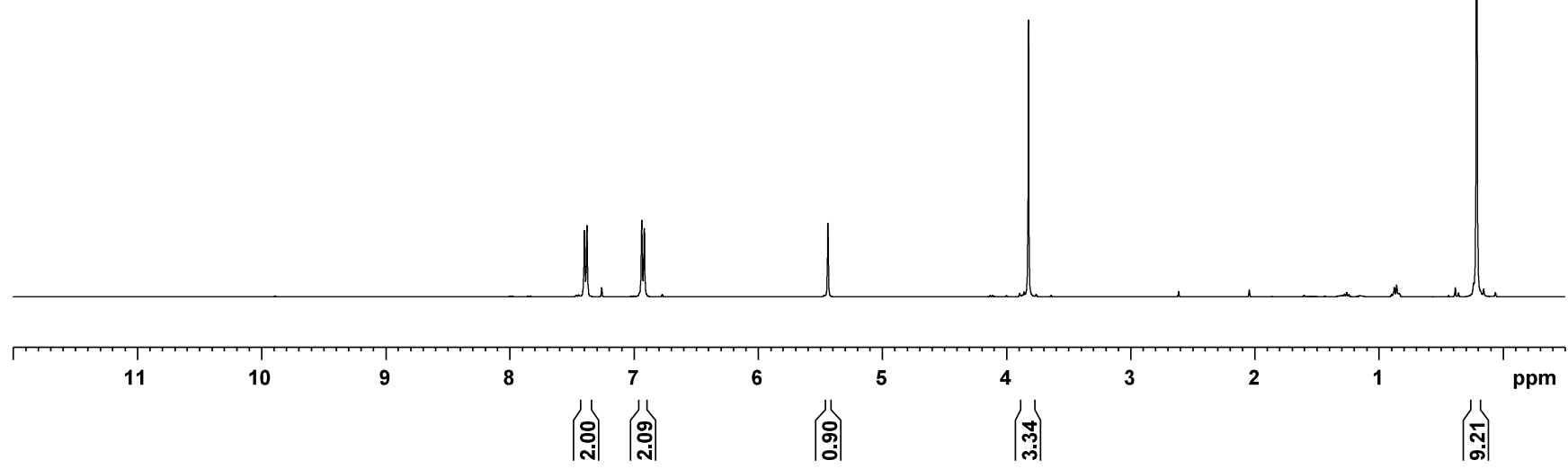



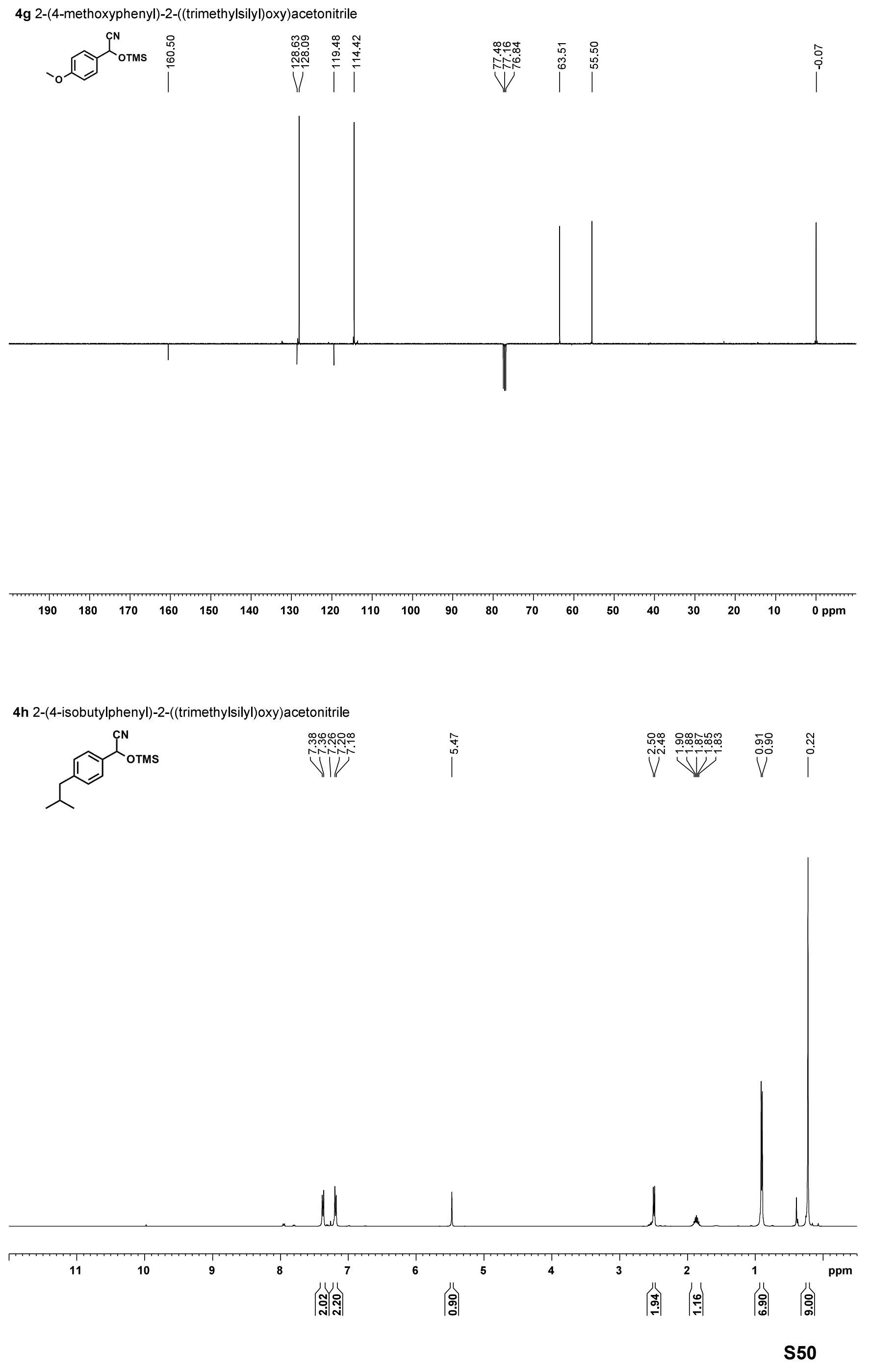
4h 2-(4-isobutylphenyl)-2-((trimethylsilyl)oxy)acetonitrile

(1) OTMs

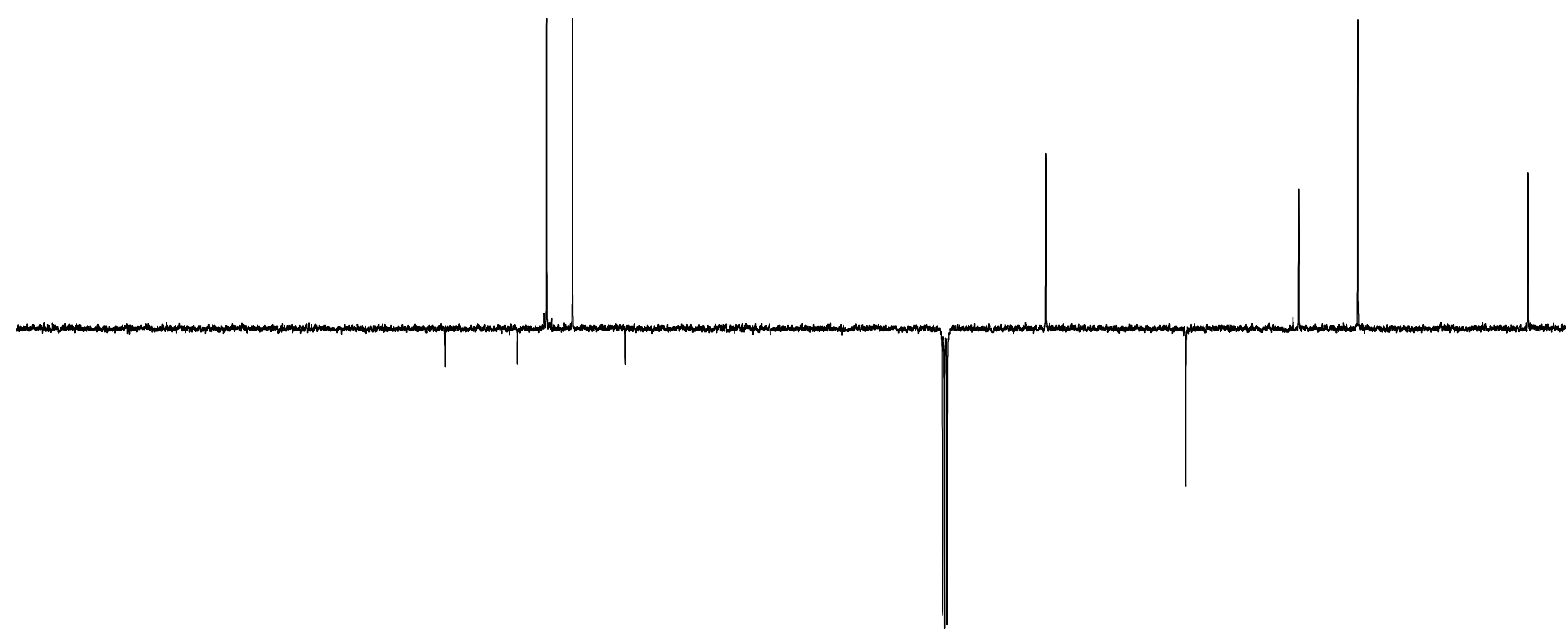

$\begin{array}{llllllllllllllllllllll}190 & 180 & 170 & 160 & 150 & 140 & 130 & 120 & 110 & 100 & 90 & 80 & 70 & 60 & 50 & 40 & 30 & 20 & 10 & \text { ppm }\end{array}$

4i 2-(furan-2-yl)-2-((trimethylsilyl)oxy)acetonitrile<smiles>CS(=O)(=O)OC(C#N)c1ccco1</smiles>

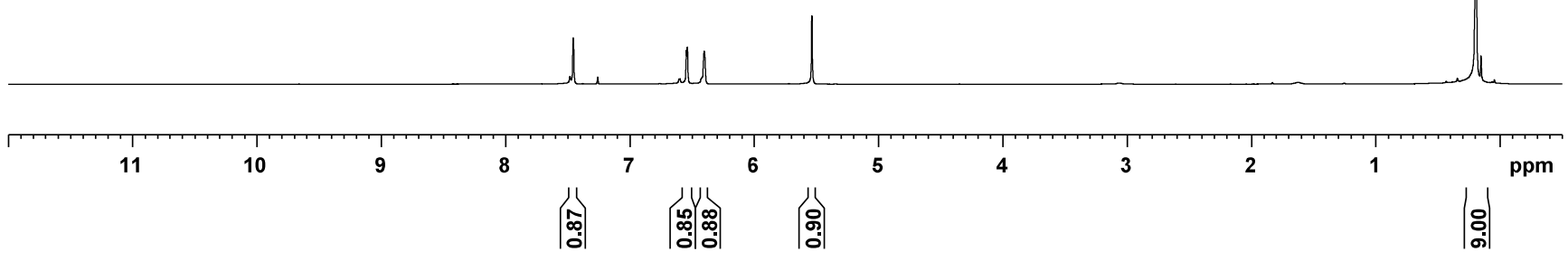



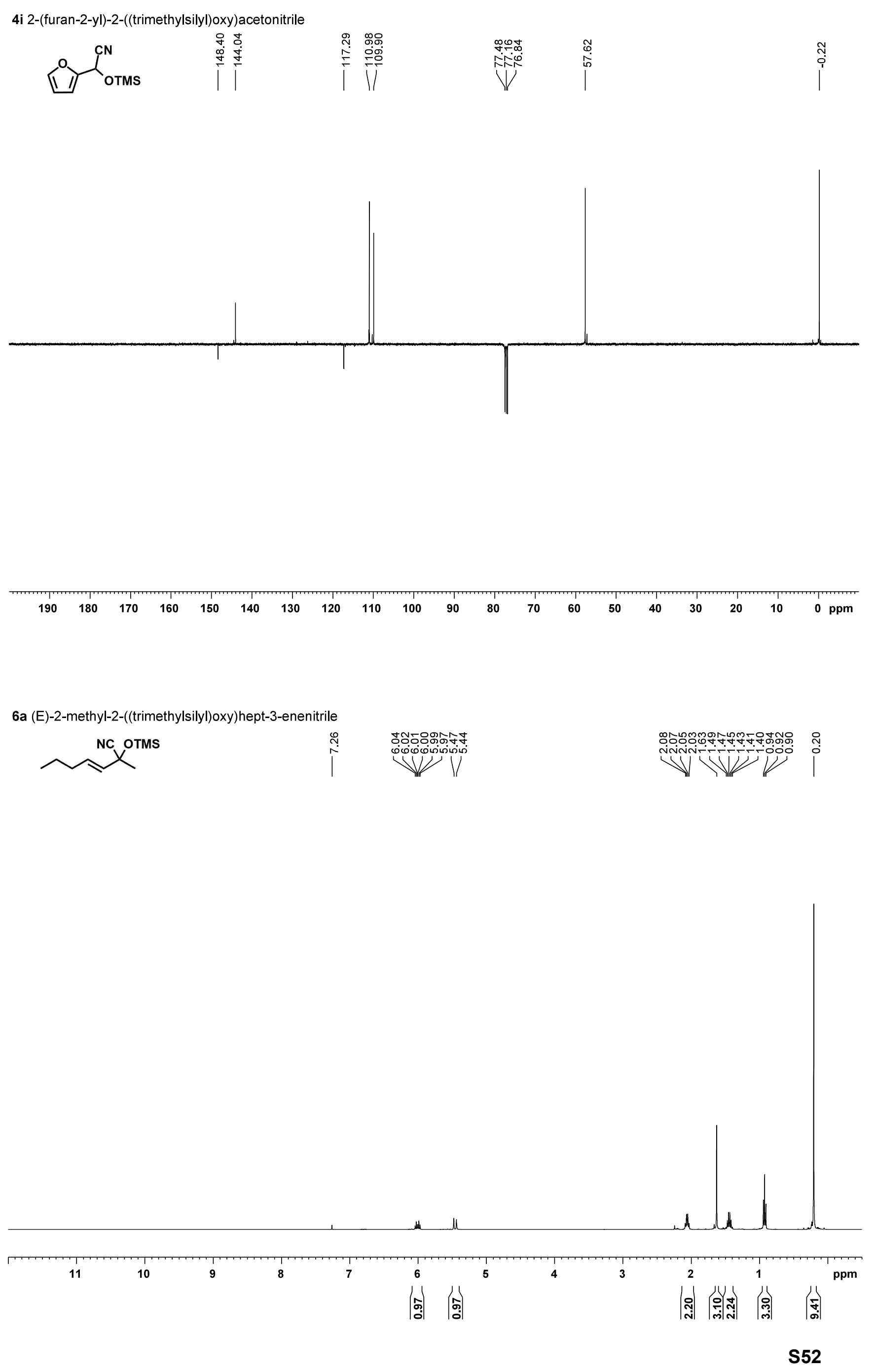
6a (E)-2-methyl-2-((trimethylsily))oxy)hept-3-enenitrile

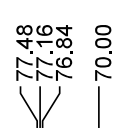

$\left.\right|_{\substack{\infty \\ \infty}} ^{\substack{\infty \\ \infty}} \stackrel{\substack{\infty \\ \infty}}{\infty}$
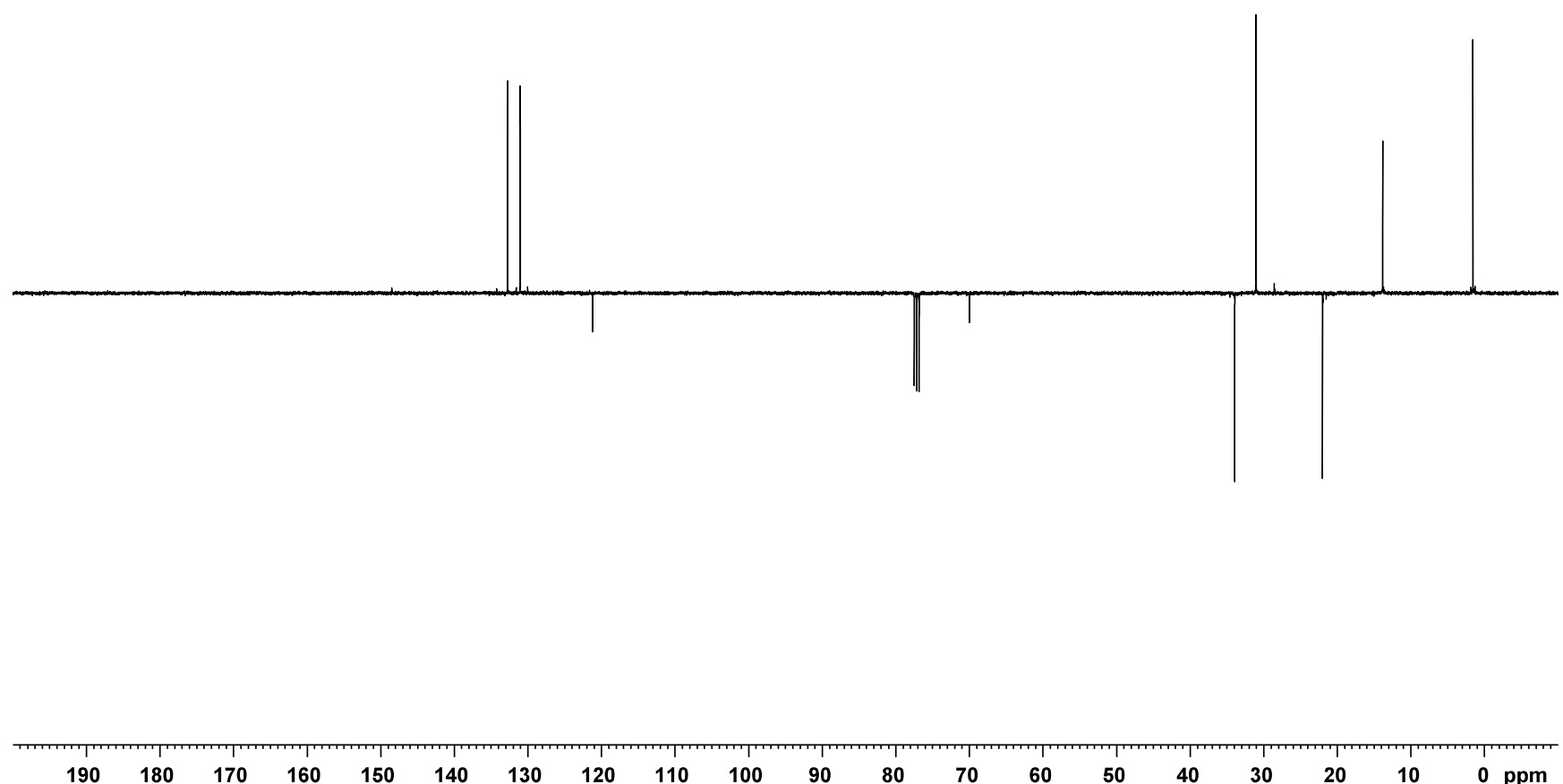

6b 2-ethyl2-((trimethylsilyl)oxy)but-3-enenitrile

$\otimes^{\text {NC OTMS }}$

-
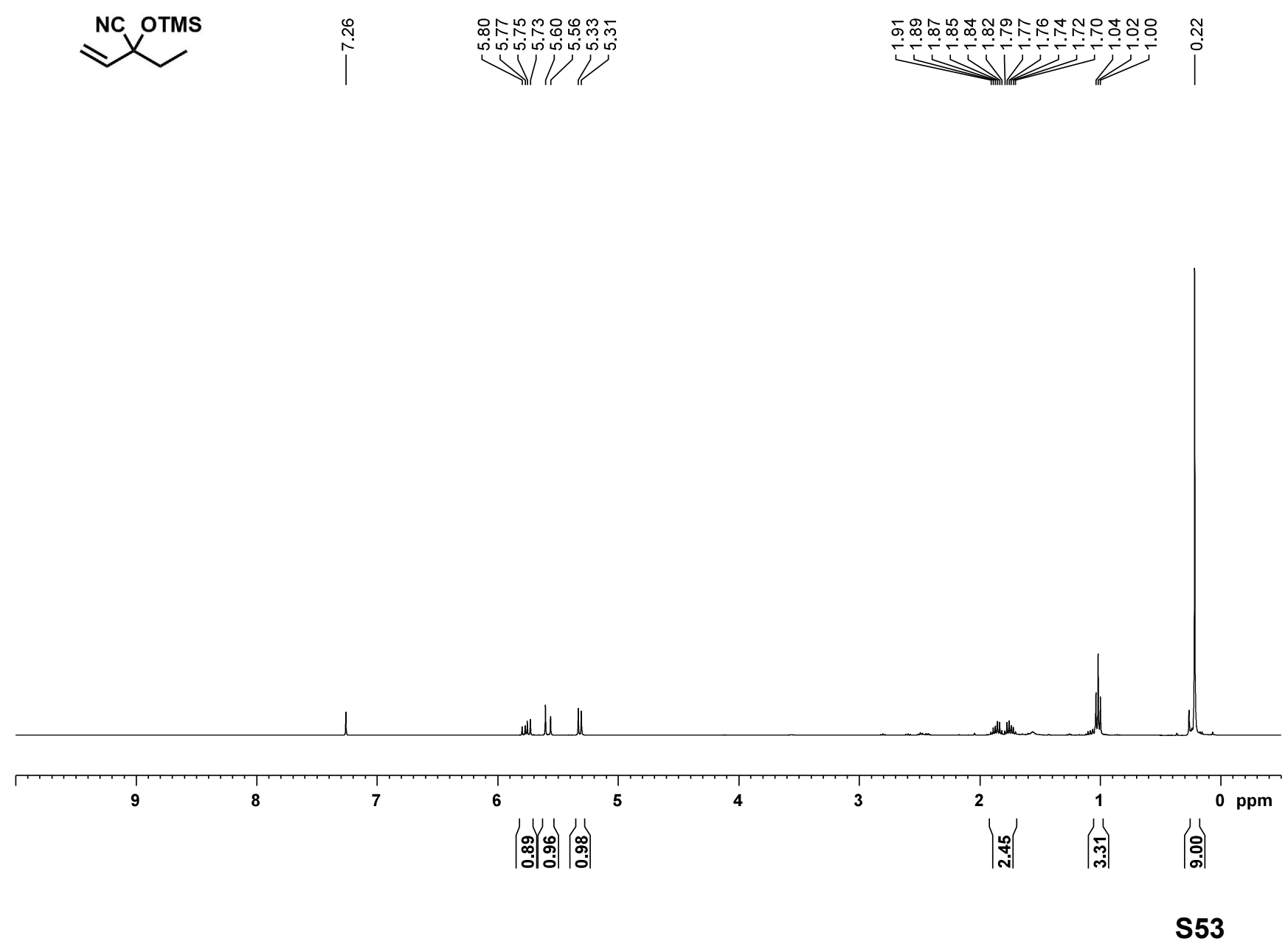


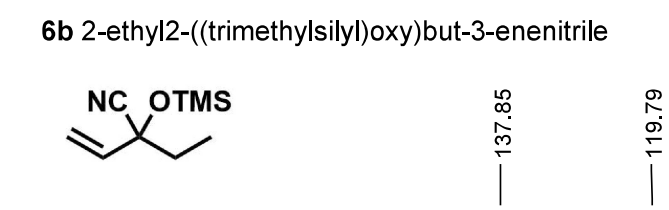
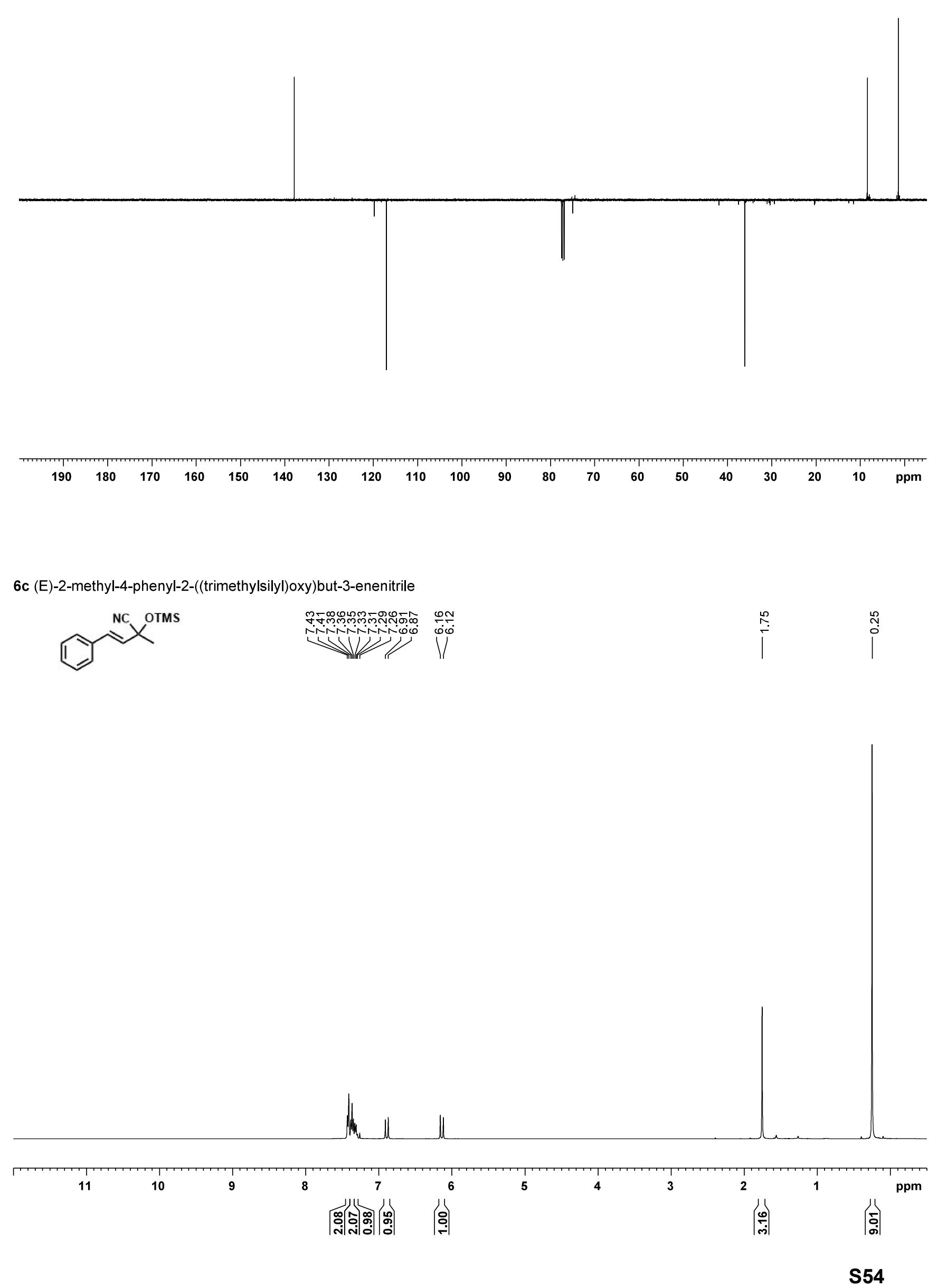
6c (E)-2-methyl-4-phenyl-2-((trimethylsilyl)oxy)but-3-enenitrile

og

TiVi |

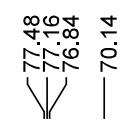

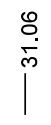

i

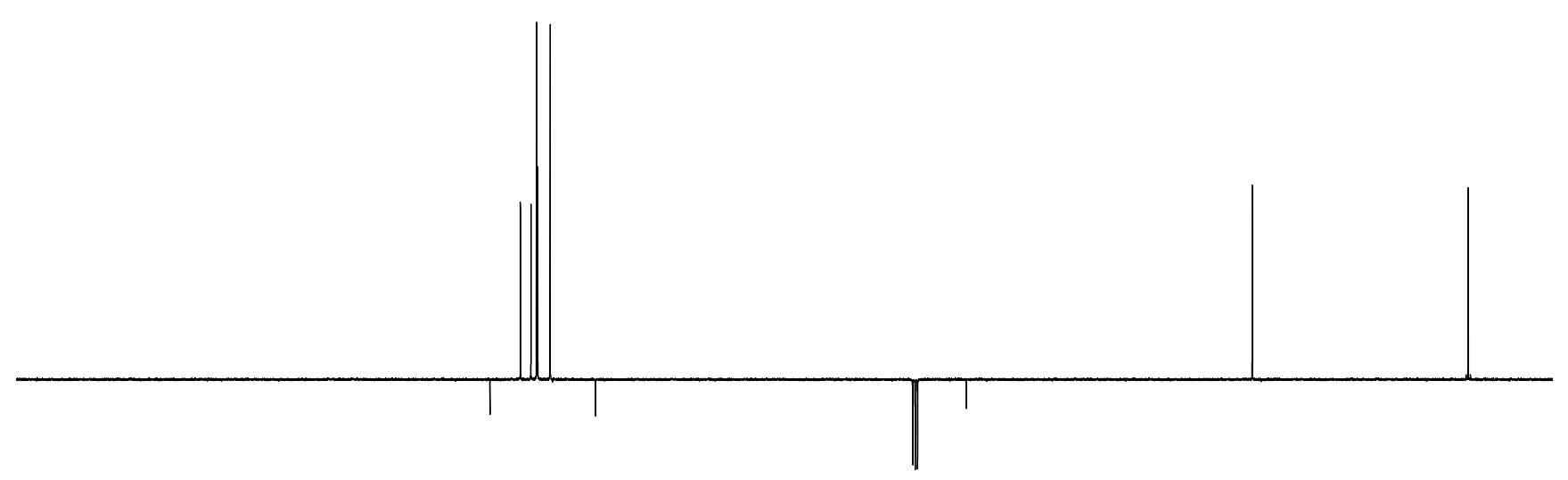

6d (E)-4-phenyl-2-((trimethylsilyl)oxy)but-3-enenitrile
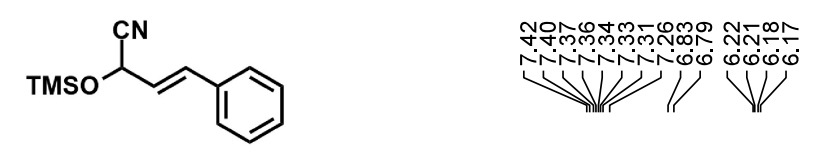

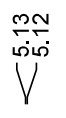

$\stackrel{\substack{0 \\ i}}{i}$

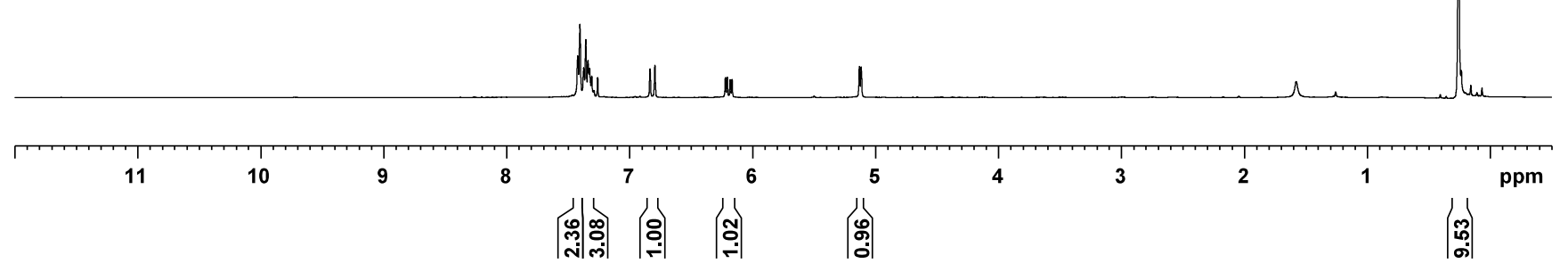

S55 
6d (E)-4-phenyl-2-((trimethylsilyl)oxy)but-3-enenitrile

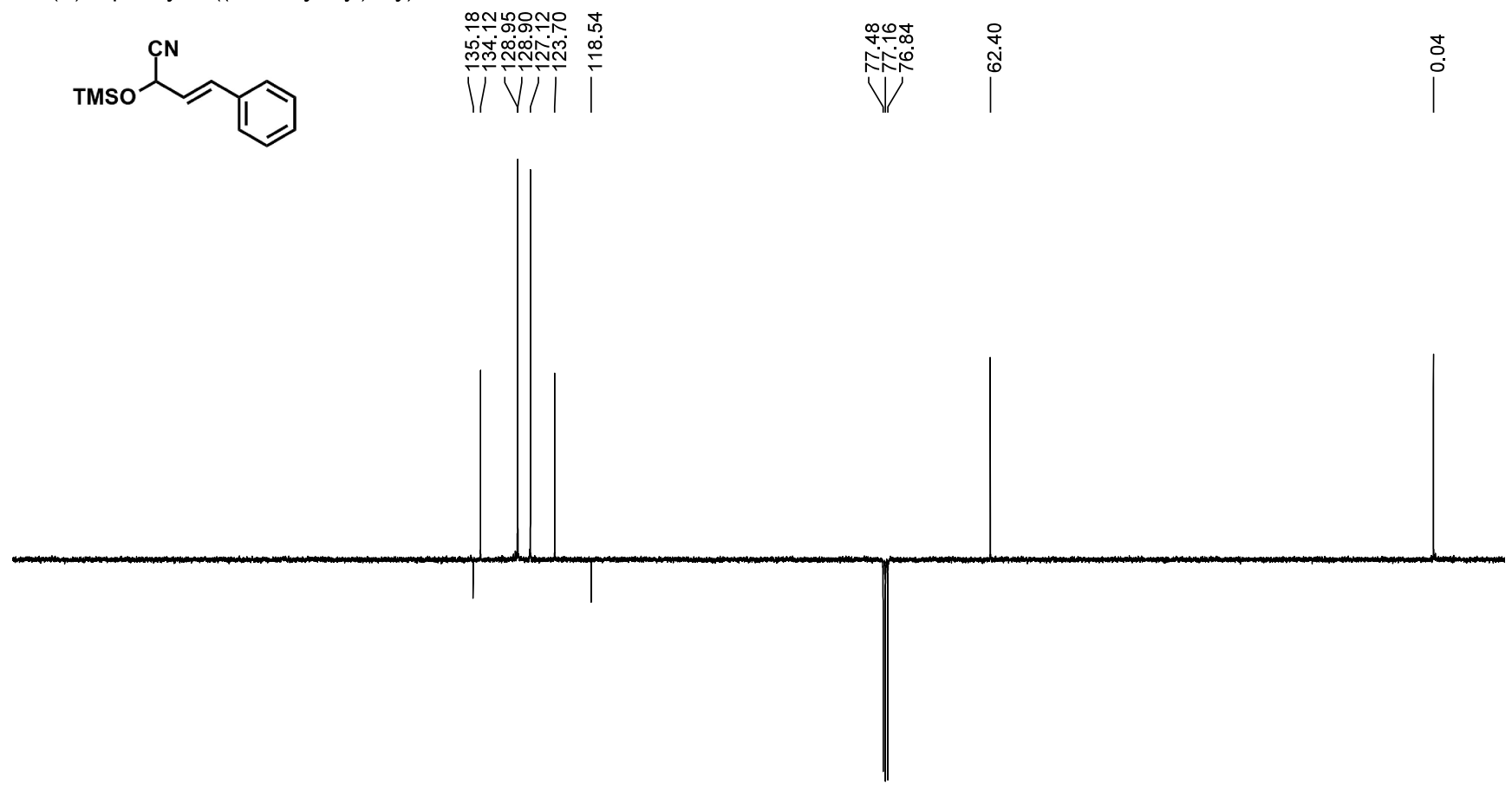

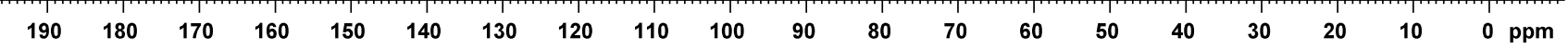

